\title{
A HUBBLE SPACE TELESCOPE SURVEY OF THE MID-ULTRAVIOLET MORPHOLOGY OF NEARBY GALAXIES ${ }^{1}$
}

\author{
Rogier A. Windhorst, Violet A. Taylor, ${ }^{2}$ Rolf A. Jansen, Stephen C. Odewahn, and \\ Claudia A. T. Chiarenza \\ Department of Physics and Astronomy, Arizona State University, Box 871504, Tempe, AZ 85287-1504; \\ rogier.windhorst@asu.edu \\ Christopher J. CONSELICE 3 \\ Department of Astronomy, Caltech, MS 105-24, Pasadena, CA 91125
}

RICHARD DE GRIJS

Institute of Astronomy, Madingley Road, University of Cambridge, Cambridge CB3 0HA, UK

Roelof S. DE JonG ${ }^{4}$ and John W. MacKenty

Space Telescope Science Institute, 3700 San Martin Drive, Baltimore, MD 21218

PAUL B. ESKRIDGE ${ }^{2}$

Department of Physics and Astronomy, Minnesota State University, Mankato, MN 56003

JAY A. FrogEL 2,5

Code S, NASA Headquarters, 300 D Street SW, Washington, DC 20546

JOHN S. GALLAGHER III ${ }^{3}$

Department of Astronomy, University of Wisconsin, 475 North Charter Street, Madison, WI 53706-1582

JOHN E. HiBBARD ${ }^{3}$

National Radio Astronomy Observatory, 520 Edgemont Road, Charlottesville, VA 22903-2475

LYNN D. MATTHEWs ${ }^{3}$

Center for Astrophysics, 60 Garden Street, Cambridge, MA 02138

AND

ROBERT W. O'CONNELL

Department of Astronomy, University of Virginia, P.O. Box 3818, Charlottesville, VA 22903-0818

Received 2001 July 2; accepted 2002 May 3

\begin{abstract}
We present a systematic imaging survey of 37 nearby galaxies observed with the Hubble Space Telescope (HST) Wide Field and Planetary Camera 2 (WFPC2) in the mid-UV F300W filter, centered at $2930 \AA$, as well as in the $I$-band $(\mathrm{F} 814 \mathrm{~W})$ filter at $8230 \AA$. Eleven of these galaxies were also imaged in the F255W filter, centered at $2550 \AA$. Our sample is carefully selected to include galaxies of sufficiently small radius and high predicted mid-UV surface brightness to be detectable with WFPC2 in one orbit and covers a wide range of Hubble types and inclinations. The mid-UV (2000-3200 §) spans the gap between ground-based $U B V$ $R(I J H K)$ images, which are available or were acquired for the current study, and far-UV images available from the Astro/UIT missions for 15 galaxies in our sample. The first qualitative results from our study are as follows:

1. Early-type galaxies show a significant decrease in surface brightness going from the red to the mid-UV, reflecting the absence of a dominant young stellar population and in some cases the presence of significant (central) dust lanes. Galaxies that are early types in the optical show a variety of morphologies in the mid-UV that can lead to a different morphological classification, although not necessarily as later type. Some earlytype galaxies become dominated by a blue nuclear feature or a point source in the mid-UV, e.g., as a result of the presence of a Seyfert nucleus or a LINER. This is in part due to our mid-UV surface brightness selection, but it also suggests that part of the strong apparent evolution of weak AGNs in early-type galaxies may be due to surface brightness dimming of their UV-faint stellar population, which renders the early-type host galaxies invisible at intermediate to higher redshifts.

2. About half of the mid-type spiral and star-forming galaxies appear as a later morphological type in the mid-UV, as Astro/UIT also found primarily in the far-UV. Sometimes these differences are dramatic (e.g.,

\footnotetext{
${ }^{1}$ Based on observations made with the NASA/ESA Hubble Space Telescope, obtained at the Space Telescope Science Institute (STScI), which is operated by the Association of Universities for Research in Astronomy (AURA), Inc., under NASA contract NAS 5-26555. Also based in part on observations made with the Vatican Advanced Technology Telescope: the Alice P. Lennon Telescope and the Thomas J. Bannan Astrophysics Facility.

${ }^{2}$ Visiting Astronomer, Cerro Tololo International Observatory, National Optical Astronomy Observatory, which is operated by AURA, Inc., under cooperative agreement with the National Science Foundation (NSF).

${ }^{3}$ Visiting Astronomer, Kitt Peak National Observatory, National Optical Astronomy Observatory, which is operated by AURA, Inc., under cooperative agreement with the NSF.

${ }^{4}$ Hubble Fellow.

${ }^{5}$ Permanent address: Department of Astronomy, The Ohio State University, 140 West 18th Avenue, Columbus, OH 43210.
} 
NGC 6782 shows a spectacular ring of hot stars in the mid-UV). However, not all mid-type spiral galaxies look significantly different in the mid-UV. Their mid-UV images show a considerable range in the scale and surface brightness of individual star-forming regions. Almost without exception, the mid-type spirals in our sample have their small bulges bisected by a dust lane, which often appears to be connected to the inner spiral arm structure.

3. The majority of the heterogeneous subset of late-type, irregular, peculiar, and merging galaxies display F300W morphologies that are similar to those seen in F814W, but with important differences due to recognizable dust features absorbing the bluer light and to hot stars, star clusters, and star formation " ridges" that are bright in the mid-UV. Less than one-third of the galaxies classified as late type in the optical appear sufficiently different in the mid-UV to result in a different classification.

Our HST mid-UV survey of nearby galaxies shows that, when observed in the rest-frame mid-UV, early- to mid-type galaxies are more likely to be misclassified as later types than late-type galaxies are to be misclassified as earlier types. This is because the later type galaxies are dominated by the same young and hot stars in all filters from the mid-UV to the red and so have a smaller " morphological $K$-correction" than true earlier type galaxies. The morphological $K$-correction can thus explain part, but certainly not all, of the excess faint blue late-type galaxies seen in deep HST fields.

Subject headings: galaxies: elliptical and lenticular, $\mathrm{cD}$ — galaxies: interactions — galaxies: irregular — galaxies: peculiar — galaxies: spiral — ultraviolet: galaxies

On-line material: additional figures

\section{INTRODUCTION}

\subsection{The Need for Reliable Classification of Faint Optical Galaxy Samples}

Since the mid-1990s, faint galaxies have been observed with the Hubble Space Telescope (HST). The best statistics, spatial sampling, and areal coverage are currently achieved in the $I$ band $(\mathrm{F} 814 \mathrm{~W})$. Good HST morphological information is available today for $\sim 10^{5}$ galaxies with $I \lesssim 26$ mag. These galaxies come from the two Hubble Deep Fields (HDFs; Williams et al. 1996, 2000), their flanking fields, the HST Medium Deep Survey, and other HST parallel surveys (e.g., Griffiths et al. 1994; Driver, Windhorst, \& Griffiths 1995a; Driver et al. 1995b; Odewahn et al. 1996; Abraham et al. 1996, 1999; Roche et al. 1997; Windhorst et al. 1997; Cohen et al. 2002).

The median half-light radius of faint field galaxies with $I \sim 26 \mathrm{mag}$ is $r_{e} \simeq 0$ ". $2-0$ ". 3 (Odewahn et al. 1996). Because of the high spatial resolution required for proper morphological classifications (e.g., using artificial neural networks), $H S T$ 's $2.4 \mathrm{~m}$ aperture limits the useful wavelength for quantitative galaxy classification to $4000 \AA \lesssim \lambda \lesssim 8000 \AA$. NICMOS $H$-band images $(\sim 0$ ". 16 FWHM) do not necessarily sufficiently resolve these faint galaxies to accurately measure their half-light radii and other important parameters.

For $I \lesssim 23 \mathrm{mag}$, a few hundred galaxies have their morphology measured at HST resolution and have measured spectroscopic redshifts plus velocity dispersion or rotation curves (e.g., Cohen et al. 2000; Lilly et al. 1998; Simard et al. 1999; Vogt et al. 1997), leading to more quantitative studies of their physical properties such as mass and $M / L$ ratios. However, because morphology can be determined with relative ease with HST for a very large number of galaxies, this ability allows us to explore a part of parameter space that constrains galaxy formation and evolution and that is not yet accessible to satisfactory spectroscopic study and thus lacks kinematic data for mass measurements.

The most dramatic result from these HST morphological studies in the mid- to late 1990s was that at faint fluxes, latetype/irregular galaxies completely dominate the faint blue galaxy counts (see Driver et al. 1995a, 1995b, 1998; Glazebrook et al. 1995; Odewahn et al. 1996; Windhorst et al.
1996, 1998b, 1999). A number of authors interpreted this result as evidence for the existence of a population of starforming galaxies that underwent substantial evolution since $z \lesssim 3$ (Ellis et al. 1996; Pascarelle et al. 1996; Driver et al. 1998; Abraham et al. 1999). Elliptical galaxies and earlytype spirals, on the other hand, have evolved much less since $z \lesssim 1$ (see Driver et al. 1995b; Lilly et al. 1998; Cohen et al. 2002). The photometric redshift distribution as a function of observed $I$-band morphological type, $N\left(z_{\text {phot }}\right.$, type $)$, suggests a differential evolution of galaxies as a function of type (Driver et al. 1998), implying a gradual formation of the Hubble sequence with cosmic time.

In the deepest $H S T$ fields, where morphological classifications are achievable to $I \lesssim 26 \mathrm{mag}$ (e.g., Odewahn et al. 1996; Driver et al. 1998), the sampled redshift range is $z \simeq 1-3$, while the bulk of the galaxies is at $z \lesssim 2$. Faint highredshift galaxies observed in the $I$ band are therefore primarily seen in the rest-frame mid-UV wavelength range, or 2000-3200 A. The reliability of results derived from the faint HST galaxy morphologies is therefore fundamentally limited by the uncertain rest-frame mid-UV morphology of nearby galaxies. For instance, although these faint, late-type/irregular objects resemble some classes of nearby late-type and peculiar galaxies (e.g., Hibbard \& Vacca 1997), they need not be physically late-type objects. Instead, they may be earlier type galaxies that look dramatically different in the rest-frame UV. Because of the wavelength dependence of nearby galaxy morphology (especially toward the UV), the outcome of faint galaxy classifications will depend on the rest-frame wavelength sampled. This "morphological K-correction" can be quite significant, even between the $B$ and near-IR bands (e.g., Knapen \& Beckman 1996), and must be quantified in order to distinguish genuine evolutionary effects from simple bandpass shifting.

The mid-UV is the optimal wavelength region for comparing the galaxies seen with $H S T$ in the $I$ band at $z \gtrsim 2$ to nearby samples and to address the question of whether the numerous late-type objects that dominate the faint blue galaxy counts are truly new classes of objects or just reflect the redshifted UV morphology of ordinary galaxies as seen nearby. In the mid-UV, one currently attains the highest res- 
olution plus largest field of view (FOV) with the HST Wide Field and Planetary Camera 2 (WFPC2).

In this paper we therefore present a systematic WFPC2 mid-UV imaging survey for a representative sample of 37 nearby galaxies. Having multicolor observations for both nearby and distant objects at the same rest-frame wavelengths will allow us to calibrate the dependence of morphology on rest-frame wavelength and thus to improve our ability to interpret the morphology of distant galaxies. Such a survey will also help us better understand the physical drivers of the rest-frame mid-UV emission, i.e., the relation between star formation and the global physical characteristics of galaxies, their recent star formation history, and the role of dust absorption and scattering.

\subsection{UV Morphology of Nearby Galaxies as a Benchmark for High-Redshift Classifications}

Observations of high-redshift galaxies in the optical spectral region detect light that was emitted as UV luminosity in these galaxies' rest frames. They therefore primarily trace high surface brightness (SB) regions populated by high densities of young $(\lesssim 1 \mathrm{Gyr})$ hot stellar populations. The dominance of young stellar complexes in producing UV light from star-forming galaxies was recognized from early satellite UV photometric observations (e.g., Code \& Welch 1982; Israel, de Boer, \& Bosma 1986), IUE far-UV spectra (e.g., Kinney et al. 1993 and references therein), as well as imaging of a few key objects from sounding rockets (e.g., Carruthers, Opal, \& Heckathorn 1978; Stecher et al. 1982). These results were confirmed when UV images became available from the FOCA balloon observations (Milliard et al. 1992) and the Astro/UIT flights. The latter imaged $~ 100$ nearby galaxies in the far-UV $(1500 \AA)$ and $\sim 40$ in the midUV $\left(2500 \AA\right.$ ) at a resolution of $\sim 3^{\prime \prime}$ FWHM (Bohlin et al. 1991; Hill et al. 1992; Kuchinski et al. 2000; Marcum et al. 2001). Morphological $K$-corrections derived from these UIT images (and from earlier sounding rocket data) were first investigated by Bohlin et al. (1991) and Giavalisco et al. (1996). This, as well as the FOCA data, showed that galaxy morphology changes quite dramatically below $3600-4000$ $\AA$, where the hot (young) stellar population, located mainly in spiral arms and $\mathrm{H}$ II regions, dominates the spectral energy distribution (SED) and where bulges essentially disappear (see also Burgarella et al. 2001; Kuchinski et al. 2001). Galaxies therefore often appear to be of later Hubble type the further one looks into the rest-frame UV. Qualitatively, this is easy to understand: in the optical/near-IR, we see the accumulated luminous phases of long-lived ( $>1 \mathrm{Gyr}$ ) stars, which emit most of their energy at longer wavelengths, whereas the mid-UV samples the star formation rate (SFR) averaged over the past gigayear or less. The mid-UV includes the longest wavelengths where young stars can dominate the integrated galaxy light and traces primarily presently active star-forming regions, or those regions where star formation has only recently shut down.

Far-UV images of nearby early-type galaxies are not always appropriate for high-redshift comparisons, since they may be affected by the "UV upturn," a spectral feature that is likely caused by hot, low-mass, old stars (O'Connell et al. 1992; O'Connell 1999) and that only appears at relatively recent epochs $(z \lesssim 0.3$; Burstein et al. 1988; Greggio \& Renzini 1990; Brown et al. 2000). However, middle-aged stellar systems can be very dim in the UV compared to either older or younger objects, as in the case in, e.g., M32. In galaxies of all types the far-UV to mid-UV emission can also be modulated by dust.

\subsection{Other Ways of Addressing the Morphological K-Correction}

A number of authors have explored the effects of bandpass shifting using multicolor optical images to extrapolate to the rest-frame UV on a pixel-by-pixel basis. This has been done using ground-based data (e.g., Hibbard \& Vacca 1997; Brinchmann et al. 1998), FOCA mid-UV images (Burgarella et al. 2001), UIT far-UV images (Kuchinski et al. 2000), and HST images of galaxies at moderate redshifts (Abraham et al. 1999; Bouwens, Broadhurst, \& Silk 1998). The peculiarities in shape and size distributions measured in deep HST surveys are found to considerably exceed the effects of bandpass shifting. While this is a robust result, these methods may not capture the full range of rest-frame UV galaxy SEDs and are less suitable for making detailed comparisons with the local universe.

For instance, there is considerable scatter in the mid-UV for galaxies of a given optical color. Donas, Milliard, \& Laget (1995) find a 3 mag range in (UV-B) colors at a given $(B-R)$ color in a faint space-based galaxy sample, where a 2000 A filter was used to measure the UV flux. This UV/ optical decoupling is confirmed by the spectroscopy of Treyer et al. (1998) and implies that the true evolutionary history of galaxies can be very different from that inferred from optical data alone and that mid-UV observations are necessary for a better understanding.

Recent observations of the Hubble Deep Field-North with $H S T /$ NICMOS show that some galaxies out to $z \sim 2.5$ appear "regular" and fit into the Hubble sequence in the near-IR, but look more patchy and irregular at shorter wavelengths (Dickinson et al. 2001). Galaxies that look disturbed in the optical, on the other hand, in general also look very similar in the rest-frame UV. These distorted high- $z$ galaxies also tend to be high-SB objects in the UV, forming stars at a high rate. Nearby galaxies whose structure is dominated by star formation also have similar morphologies in the rest-frame optical and mid-UV (e.g., Conselice et al. 2000), which suggests that some nearby starburst galaxies may be analogs to these "peculiar" high- $z$ objects.

\subsection{Goals of Our HST Mid-UV Imaging Survey}

In this section we briefly summarize the main science goals of our project, which determine the size and properties of our WFPC2 mid-UV sample. We aim to (1) provide local benchmark images that we can artificially redshift to $z \simeq 1-3$ for quantitative comparison to the morphology and photometric properties of high-redshift galaxies; (2) consistently classify polychromatic structures within galaxies from 0.2 to $2 \mu \mathrm{m}$ with artificial neural networks; (3) map the spatial distribution, luminosities, and sizes of star-forming regions dominating the UV morphology and relate these to global galaxy properties; and (4) map the effects of dust at mid-UV wavelengths in galaxies as a function of type, inclination, and galactocentric radius. In the present paper we present the benchmark mid-UV images and a qualitative discussion of our results in the context of these science goals. More quantitative studies will be presented in subsequent papers by members of our mid-UV team. 
This paper is organized as follows. In $\S 2$ we outline our survey strategy, describe the sample selection, and discuss the WFPC 2 observations. We also discuss the data processing and calibration. Our WFPC2 mid-UV and groundbased optical images are presented in $\S 3$, where we also discuss individual objects. We describe the general trends in galaxy morphology as observed from the mid-UV through the optical in $\S 4$. In $\S 5$ we present our main conclusions from this data set.

\section{STRATEGY, SAMPLE SELECTION, AND OBSERVATIONS}

\subsection{WFPC2 Mid-UV Survey Strategy}

\subsubsection{Mid-UV Filters}

We have obtained images of 37 nearby galaxies with $H S T$ /WFPC2 through one or, whenever possible, two wide-band mid-UV filters below the atmospheric cutoff. These filters are F300W $\left(\lambda_{\text {cent }} \simeq 2930 \AA ; \Delta \lambda \simeq 740 \AA\right.$ FWHM $)$ and F255W $\left(\lambda_{\text {cent }} \simeq 2550 \AA ; \quad \Delta \lambda \simeq 395 \AA\right.$ FWHM), which provide reasonable red-leak suppression. The F255W, F300W, and Johnson $U$ and $B$ filters are approximately equally spaced in energy (i.e., in the logarithm of the wavelength), and so add significantly to the existing ground-based optical-near-IR color baseline.

Since the $H S T /$ WFPC2 system throughput is $\sim 2.0 \%$ in F300W and $\sim 0.5 \%$ in F255W (Biretta et al. 2001, Appendix 1 ), we can only detect the highest SB, bluest objects in F255W in a single $H S T$ orbit, and so have selected our sample accordingly. The mid-UV is the longest wavelength where younger stars can dominate the integrated galaxy light and therefore the regime of choice to measure the SFR averaged over $\lesssim 1$ Gyr. We have observed all selected galaxies through the F300W filter, spending no more than one full orbit per galaxy. In that same orbit a short exposure through a red filter (F814W) is taken for adequate red-leak correction (see $\S 2.4 .3$ ). For galaxies in the HST continuous viewing zone (CVZ), we also took exposures in the F255W filter (see $\S 2.1 .4)$.

\subsubsection{Predicted Mid-UV Surface Brightness}

We predict the average mid-UV SB, $\mu_{\mathrm{F} 300 \mathrm{~W}}$, for a given galaxy from its total $B$ magnitude $B_{T}$, its $(U-B)$ color, its half-light radius $r_{e}$, and its ellipticity $b / a$ (as tabulated in or derived from the RC3 catalogue [de Vaucouleurs et al. 1991] or the NASA/IPAC Extragalactic Database [NED]), as follows:

$$
\mu_{\mathrm{F} 300 \mathrm{~W}}=\mathrm{F} 300 \mathrm{~W}_{T}+0.75+2.5 \log \left(\pi r_{e}^{2} \frac{b}{a}\right),
$$

i.e., half the total predicted $\mathrm{F} 300 \mathrm{~W}$ magnitude, $\mathrm{F} 300 \mathrm{~W}_{T}$, within the effective area. We used the updated Bruzual \& Charlot (1993) models to transform the $(U-B)$ color for each galaxy type to a predicted $(\mathrm{F} 300 \mathrm{~W}-B)$ color, from which $\mathrm{F} 300 \mathrm{~W}_{T}$ follows. To a reasonable approximation we find $(\mathrm{F} 300 \mathrm{~W}-B) \simeq 2(U-B)$. In the absence of a $(U-B)$ color, a prediction for $(U-B)$ was made from the measured $(B-V)$ color and the known $(U-B)$ versus $(B-V)$ relation for RC3 galaxies as a function of galaxy type (de Vaucouleurs et al. 1991). Our sample was selected to have $18 \mathrm{mag} \operatorname{arcsec}^{-2} \lesssim \mu_{\mathrm{F} 300 \mathrm{~W}} \lesssim 22.5-23.0 \mathrm{mag} \operatorname{arcsec}^{-2}$. For this range in $\mathrm{SB}$, a galaxy can be detected out to $r \simeq 2-3 r_{e}$ with WFPC2 in one orbit with sufficiently high signalto-noise ratio $(\mathrm{S} / \mathrm{N})$ to allow morphological features to be recognized.

The bias toward selecting higher SB galaxies can be addressed as in Driver et al. (1995a). In short, selecting high-SB galaxies as the nearby template objects is not an overriding concern, since the high-redshift samples are similarly biased (or more so) in favor of high-SB galaxies as a result of the severe cosmological SB dimming. For monochromatic light, SB dimming is proportional to $(1+z)^{(4+\alpha)}$, with $\alpha$ the spectral index if the object spectrum were to be represented by a power-law SED.

The resolution of HST's Optical Telescope Assembly (OTA) in F300W is $\sim 0$ ".04 (FWHM). This is somewhat larger than HST's formal diffraction limit at $2930 \AA$ $(1.22 \lambda / D \simeq 0$ ".03), which does not set in until longward of $4000 \mathrm{~A}$ as a result of mirror microroughness. The WFPC2 WFC pixel size is $\simeq 0^{\prime \prime} 0996$ pixel $^{-1}$. Hence, because the UV images are already severely undersampled, on-chip rebinning to gain SB sensitivity is not an option. Instead, where needed, we can rebin the images in the postprocessing stage to measure the outskirts to fainter SB levels. This improves the SB sensitivity (see $\S 2.4 .2$ ) in the outskirts to $\sim 25.8-26.3$ mag $\operatorname{arcsec}^{-2}$ in F300W and to $\sim 23.8-24.2 \mathrm{mag} \mathrm{arcsec}^{-2}$ in F255W, sufficient to get good light profiles for $r \lesssim 2-3 r_{e}$.

\subsubsection{Target Size and Placement inside WFPC2}

The WFPC2 FOV measures $\sim 2 ! 5$ (along the WFC CCDs). We selected the sample to fit within the FOV, and preferably within a single $75^{\prime \prime} \times 75^{\prime \prime}$ WFC CCD, allowing us to derive reliable $\mathrm{SB}$ profiles without having to mosaic multiple WFPC2 fields. For galaxies with a $B$-band half-light radius in the range $0.1 \lesssim r_{e} \lesssim 1^{\prime} 0$ (as derived from the RC3 catalogue; de Vaucouleurs et al. 1991), about $\sim 3-5$ scale lengths fit in a single WFPC2 field.

For most of our sample galaxies, the nucleus has been placed on WFPC2's WF3 CCD, near pixel $(X, Y)=$ $(300,300)$, so that both the WF2 and WF4 CCDs maximally sample the galaxies' outskirts, allowing optimal subtraction of any sky background when mosaicking the four WFPC2 CCDs. For some of the larger galaxies and for galaxies in pairs or small groups, we center the object(s) in another part of the WFPC2 FOV or constrain the HST roll angle ("ORIENT") to assure that the largest possible portion of the galaxy or galaxy group is observed.

\subsubsection{The HST Continuous Viewing Zone}

Part of the galaxy sample is located in the HST CVZ, i.e., at $53^{\circ} \leq|\delta| \leq 72^{\circ}$, where objects are observable for an entire $H S T$ orbit, typically doubling the available integration time. For many such galaxies, we were able to obtain F255W as well as F300W images without the cost of an extra $H S T$ orbit. We select the sample to maximize the fraction of galaxies in the CVZ.

Since the zodiacal background reflects the color of the Sun, the sky background will be darker in the F255W filter $\left(\sim 24.5-25.0\right.$ mag $\left.\operatorname{arcsec}^{-2}\right)$ than in the F300W filter $(\sim 24.0$ mag $\operatorname{arcsec}^{-2}$ ), partly compensating for the lower sensitivity in F 255W. Observations in the CVZ may suffer from higher sky background levels as a result of the Earth's limb (Williams et al. 1996). We minimize the probability of excessive sky levels by interspersing the exposures in the different filters using the sequence F814W, F255W, F300W, F255W, 
F300W, F814W, F255W, and F300W. This sequence ensures that never more than one F255W or F300W exposure is taken close to the Earth's limb in a full CVZ orbit and also minimizes the number of fine guidance sensor (FGS) motions needed to create a pointing dither pattern. For non-CVZ targets we use the sequence F814W, F300W, $\mathrm{F} 300 \mathrm{~W}$, and $\mathrm{F} 814 \mathrm{~W}$, to push the $\mathrm{F} 300 \mathrm{~W}$ exposures farthest from the Earth's limb.

\subsection{Sample Selection}

\subsubsection{Available Ground-and Space-based Observations}

In selecting our galaxy sample we aimed to take advantage of as much preexisting ground- and space-based UV, optical, and near-IR images as possible. In particular, high priority was given to galaxies that have been observed with UIT in the far-UV $(1500 \AA)$ and obeyed the SB and size criteria ( $\S \S 2.1 .2$ and 2.1.3). Some of these galaxies are close to the UIT detection limit, as they are minor members in galaxy groups targeted by UIT.

In addition, nearby galaxies already observed with $H S T$ / WFPC2 in the mid-UV F300W and/or F255W filter that match our criteria will be included in our final sample. When the final Cycle 9 target list was compiled in early 2000, the HST Archive contained 15 such galaxies with F300W images obtained and two more imaged in F255W. These archival observations came primarily from Cycle 4 program GO 5381 (PI A. P. Koratkar) and Cycle 6 program GO 6355 (PI M. Giavalisco). We will analyze these 17 archival mid-UV images in a separate paper (V. A. Taylor et al. 2003, in preparation). The current Cycle 9 sample is designed and must be understood to be complementary to this existing archival sample.

Over the last decade, several of us have systematically imaged from the ground a total of about 750 nearby galaxies covering all Hubble types and inclinations. The majority of these galaxies, which includes most of the galaxies imaged in the far-UV with UIT (primarily at $1500 \AA$ ), were observed in $U B V R I$, but a good fraction were observed in the near-IR $J H K$ filters as well (Eskridge et al. 2002). The database thus compiled consists of seven individual galaxy samples:

1. 86 face-on spiral galaxies of de Jong \& van der Kruit (1994).

2. 220 galaxies with Hubble types later than $\mathrm{S} 0$ from the OSU BVRJHK survey (Frogel, Quillen, \& Pogge 1996; Eskridge et al. 2000, 2002; all inclinations).

3. 113 galaxies from the Frei et al. (1996) survey in $B$ and $R$, to which we added $U$ - and $V$-band images (containing a good number of early-type galaxies; all inclinations; V. A. Taylor et al. 2003; in preparation).

4. 100 galaxies with $1500 \AA$ and 40 with $2500 \AA$ images from the Astro/UIT mission (Kuchinski et al. 2000; Marcum et al. 2001; all inclinations).

5. 48 edge-on galaxies (de Grijs \& Peletier 1997; de Grijs, Peletier, \& van der Kruit 1997).

6. 150 late-type UGC galaxies selected to be morphologically irregular, peculiar, or merging (Hibbard \& Vacca 1997; J. E. Hibbard et al. 2002, in preparation). These have a range of impact parameters (i.e., the distance between the two interacting or colliding objects).

7. 49 late-type dwarf spiral galaxies (Matthews \& Gallagher 1997) and compact, high-SB luminous blue galaxies (Gallagher, Hunter, \& Bushouse 1989; Gallagher et al. 2000 ; all inclinations).
There is some overlap among the samples. The last two sets were specifically added to provide a good number of likely local counterparts of the peculiar and irregular galaxies seen with $H S T$ in large numbers at high redshifts. The $U B V$ $R I+J H K$ images from these ground-based surveys, where available, are included in the data presented in $\S 3$ (Fig. 4).

\subsubsection{Required Statistics}

To date, the HST Archive contains over 10,000 orbits of data on a multitude of distant galaxy projects (including parallels). To match these numerous observations of distant galaxies, we need a statistically significant and representative comparison sample of nearby galaxies that includes all morphological types. To cover a broad range of nearby galaxies, we will use three broad bins of Hubble types: (1) early-type galaxies (E-S0), (2) early- to mid-type spiral galaxies $(\mathrm{Sa}-\mathrm{Sc})$, and (3) late-type galaxies, irregulars (Sd-Irr), plus peculiars and mergers. For statistical studies as a function of photometric parameters, we will cover a range in inclination angles in three bins of $\sin i$ : edge-on, face-on, and intermediate angles. Thus, we need about 54 galaxies in total, to populate each of the nine type-inclination bins with six galaxies to begin to average over galaxy properties.

Of the 20 galaxies with WFPC2 F300W images in the archive as of early 2000 (mostly E/S0/Sabc galaxies), about 17 are small enough to be contained within the WFPC2 FOV. To this available mid-UV sample we add here a complementary set of 37 galaxies, which we selected from the ground-based samples of about 750 galaxies described in $\S 2.2 .1$. These objects comprise 24 new irregulars, peculiars, and merging/interacting galaxies and 13 new E/S0/Sabc galaxies to complement the 17 usable galaxies available in the HST Archive.

\subsubsection{Selection of the HST/WFPC2 Mid-UV Sample}

The selection criteria for inclusion in the HST/WFPC2 sample of 37 galaxies are as follows:

1. The $B$-band half-light radius should fall within the range $0.1 \lesssim r_{e} \lesssim 1{ }^{\prime} 0$.

2. The predicted average $\mathrm{SB}$ in $\mathrm{F} 300 \mathrm{~W}$ out to $r \simeq r_{e}$ should be in the range $18 \mathrm{mag} \operatorname{arcsec}^{-2} \lesssim \mu_{\mathrm{F} 300 \mathrm{~W}} \lesssim$ 22.5-23.0 mag $\operatorname{arcsec}^{-2}$.

3. The distribution of Hubble types should be representative for nearby galaxy samples when combined with the set of archival galaxies.

4. The distribution over apparent axis ratio (and hence inclination) should resemble that of nearby galaxy samples.

5. Priority should be given first to the 15 galaxies with available UIT $1500 \AA$ images that obey the other selection criteria above, and then to galaxies with multiband groundbased images available.

6. As much as possible, select galaxies that can be scheduled in the HST CVZ, while satisfying the first five criteria. Replace candidates that are difficult to schedule by alternates.

In Table 1 we list the properties of the selected sample. Most of the table entries are taken or derived from values listed in the RC3 catalogue (de Vaucouleurs et al. 1991). Columns (2) and (3) list the coordinates of the galaxy centers as measured on the Digital Sky Survey (DSS). In a few cases of complex galaxies, merging/interacting galaxies, or galaxies in small groups, the listed coordinates refer to a 
TABLE 1

Nearby Galaxies Imaged with WFPC2 in the Mid-UV in Cycle 9

\begin{tabular}{|c|c|c|c|c|c|c|c|c|c|c|c|c|c|c|c|}
\hline \multicolumn{16}{|c|}{ Cycle 9 WFPC2 Mid-UV Sample } \\
\hline NGC $1140 .$. & 025433.43 & -100142.4 & 0.55 & 0.144 & 0.830 & 18.29 & 21.34 & -0.43 & 0.35 & 12.84 & 12.26 & 10.0 & 0.0049 & 106. & 7.95 \\
\hline ESO $418-G 008$. & 033130.58 & -301246.6 & 0.66 & 0.185 & 0.587 & 20.61 & 22.37 & -0.23 & 0.41 & 13.92 & 13.84 & 8.0 & 0.0038 & 82.4 & 6.18 \\
\hline 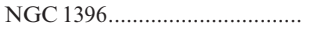 & 033806.63 & -352624.5 & 0.10 & 0.172 & 0.500 & 21.19 & 22.75 & 0.53 & & 14.80 & 16.62 & -3.0 & 0.0026 & 56.4 & 4.23 \\
\hline NGC $1510 \ldots .$. & 040332.55 & -432403.0 & 0.55 & 0.151 & 0.659 & 19.62 & 22.07 & -0.19 & 0.45 & 13.47 & 13.49 & -2.3 & 0.0028 & 60.7 & 4.55 \\
\hline IC 2184 (Mrk 8) …………............ & 072925.31 & 720739.8 & 0.78 & 0.159 & 0.416 & 19.17 & 20.51 & -0.58 & & 13.50 & 12.55 & 9.0 & 0.0124 & 269. & 20.2 \\
\hline NGC $2551 \ldots$ & 082450.16 & 732443.0 & 0.68 & 0.220 & 0.830 & 21.75 & 23.88 & 0.39 & 0.99 & 13.10 & 14.57 & 0.2 & 0.0080 & 174. & 13.1 \\
\hline UGC 05028/29 & 092756.94 & 682456.9 & 0.58 & 0.150 & 0.354 & 21.32 & 22.44 & 0.14 & $\ldots$ & 14.30 & 15.15 & 4.0 & 0.0127 & 276. & 20.7 \\
\hline UGC 05101 . & 093551.45 & 612110.5 & 0.59 & 0.183 & 0.574 & 20.10 & 21.83 & -0.89 & $\ldots$ & 15.20 & 13.47 & 11.0 & 0.0401 & 870. & 65.3 \\
\hline UGC $05189 \ldots$ & 094255.27 & 092847.9 & 0.52 & 0.226 & 0.869 & 20.22 & 22.39 & -0.73 & $\ldots$ & 14.60 & 13.27 & 10.0 & 0.0102 & 221. & 16.6 \\
\hline UGC $05626 .$. & 102427.76 & 572331.1 & 0.37 & 0.215 & 0.792 & 20.04 & 22.12 & -0.73 & & 14.90 & 57 & 10.0 & 0.0088 & 191. & 14.3 \\
\hline NGC $3310($ Arp 217)... & 103845.99 & 533010.1 & 0.78 & 0.204 & 1.545 & 17.74 & 21.38 & -0.43 & 0.35 & 11.15 & 10.57 & 4.0 & 0.0035 & 75.9 & 5.69 \\
\hline $\mathrm{MCG}+03-30-071$ & 114401.88 & 194723.7 & 0.34 & 0.162 & 0.435 & 21.42 & 2282 & -0.15 & 0.53 & 15.54 & 56 & 10.0 & 0.0163 & 354. & 26.6 \\
\hline NGC 3 & 114447.27 & 194605.3 & 1.00 & 0.134 & 0.245 & 22.02 & 22.58 & -0.12 & 0.52 & 15.30 & 15 . & 10.0 & 0.0212 & 460. & 34.5 \\
\hline NGC 3921 (Arp 224) .................... & 115106.78 & 550444.4 & 0.62 & 0.362 & 1.069 & 22.34 & 23.94 & 0.25 & 0.68 & 13.06 & 14.18 & 0.0 & 0.0198 & 430. & 32.3 \\
\hline 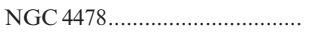 & 123017.33 & 121941.7 & 0.85 & 0.223 & 0.953 & 21.46 & 23.86 & 0.46 & 0.91 & 12.36 & 14.01 & -5.0 & 0.0044 & 95.4 & 7.16 \\
\hline IC 3949. & 125852.29 & 274921.6 & 0.19 & 0.174 & 0.512 & 21.41 & & 0.21 & 0.84 & 15.10 & 2 & -2.0 & 0.0249 & 540. & 40.5 \\
\hline UGC 08335 (Arp 238) …….......... & 131532.91 & 620736.6 & 0.89 & 0.167 & 0.467 & 22. & 23.95 & 0.04 & & 15.00 & 15.60 & 4.0 & 0.0312 & 677. & 50.8 \\
\hline Mrk $66 \ldots$ & 132553.68 & 571516.4 & 0.65 & 0.130 & 0.218 & 19.84 & 20.21 & -0.66 & 0.02 & 15.00 & 13.85 & 11.0 & 0.0221 & 479. & 35.9 \\
\hline NGC $5253 \ldots \ldots \ldots \ldots \ldots \ldots \ldots \ldots \ldots$ & 133955.76 & -313838.5 & 0.39 & 0.379 & 2.506 & 18.52 & 21.88 & -0.24 & 0.43 & 10.87 & 10.77 & 10.0 & 0.0009 & 19.5 & 1.46 \\
\hline )........... & 134139.96 & 554012.4 & 0.71 & 0.197 & 0.674 & 21.43 & 23.35 & 0.14 & $\ldots$ & 13.60 & 14.45 & 3.0 & 0.0255 & 553. & 41.5 \\
\hline 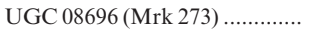 & 134442.29 & 555311.1 & 0.23 & 0.183 & 0.574 & 18.95 & 20.68 & -0.89 & $\ldots$ & 15.07 & 13. & 11.0 & 0.0380 & 824. & 61.8 \\
\hline UGC 08823 (Mrk 279) ……....... & 135303.55 & 691830.3 & 0.59 & 0.162 & 0.435 & 22.65 & 24.05 & 0.49 & $\ldots$ & 14.57 & 16.29 & -2.0 & 0.0311 & 675. & 50.6 \\
\hline 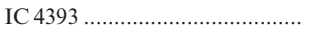 & 141749.07 & -312055.5 & 0.11 & 0.275 & 1.199 & 20.28 & 22.73 & -0.18 & $\ldots$ & 14.55 & 14.60 & 6.0 & 0.0087 & 189. & 14.2 \\
\hline UGC 09 & 152504.62 & 661515.8 & 0.33 & 0.210 & 0.757 & 19.77 & 21.80 & -0.73 & $\ldots$ & 14.80 & 13.47 & 10.0 & 0.0122 & 265. & 19.9 \\
\hline$G C 10$ & 154841.22 & 215210.1 & 0.15 & 0.275 & 1.199 & 21. & 2 & 0.04 & $\ldots$ & 14.80 & 15.40 & 4.0 & 0.0075 & 163. & 12.2 \\
\hline NGC $6789 \ldots .$. & 191641.93 & 635820.8 & 0.78 & 0.193 & 0.644 & 19.42 & 21.28 & -0.73 & 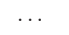 & 13.70 & 12.37 & 10.0 & 0.0005 & 10.8 & 0.81 \\
\hline NGC $6782 .$. & 192357.96 & -595521.6 & 0.66 & 0.354 & 1.094 & 21.32 & 23.02 & 0.32 & 0.92 & 11.84 & 13.14 & 0.8 & 0.0127 & 276. & 20.7 \\
\hline NGC $7685 \ldots$ & 233033.35 & 035408.4 & 0.76 & 0.489 & 0.931 & 23.37 & 24.02 & 0.00 & 0.66 & 13.84 & 14.34 & 5.3 & 0193 & 419. & 31.4 \\
\hline NGC 7769 (Mrk 9005) .. & 235103.96 & 200900.0 & 0.95 & 0.220 & 0.830 & 21.16 & 23.29 & 0.14 & & 12.77 & 13.62 & 3.0 & 0.0146 & 317. & 23.8 \\
\hline
\end{tabular}

\begin{tabular}{|c|c|c|c|c|c|c|c|c|c|c|c|c|c|c|c|}
\hline \multicolumn{16}{|c|}{ Archival WFPC2 Mid-UV Sample } \\
\hline NGC $1326 \ldots . .$. & 032356.40 & -362750.0 & 0.65 & 0.416 & 1.945 & 21.12 & 23.72 & 0.28 & 0.87 & 11.41 & 12.61 & -1.0 & 0.0041 & 88.9 & 6.67 \\
\hline NGC $1380 \ldots \ldots \ldots$. & 033626.90 & -345833.0 & 0.66 & 0.659 & 2.393 & 22.02 & 24.07 & 0.45 & 0.94 & 10.87 & 12.49 & -2.0 & 0.0057 & 124. & 9.30 \\
\hline 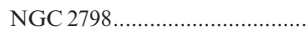 & 091722.90 & 420002.0 & 1.00 & 0.199 & 1.285 & 20.89 & 24.19 & -0.01 & 0.72 & 13.04 & 13.51 & 1.0 & 0.0058 & 126. & 9.45 \\
\hline 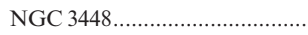 & 105439.10 & 541824.0 & 0.76 & 0.388 & 2.812 & 21.03 & 24.59 & -0.19 & 0.43 & 12.48 & 12.50 & 90.0 & 0.0047 & 102. & 7.65 \\
\hline NGC $3516 \ldots$ & 110647.30 & 723412.0 & 0.89 & 0.190 & 0.869 & 20.00 & 22.55 & -0.06 & 0.81 & 12.50 & 12.85 & -2.0 & 0.0092 & 200. & 15.0 \\
\hline NGC $4564 \ldots .$. & 123627.00 & 112621.0 & 0.36 & 0.330 & 1.774 & 21.07 & 23.97 & 0.46 & 0.93 & 12.05 & 13.70 & -5.0 & 0.0036 & 78.1 & 5.86 \\
\hline NGC $4639 \ldots$ & 124252.60 & 131530.0 & 0.78 & 0.489 & 1.377 & 22.00 & 23.50 & 0.08 & 0.70 & 12.24 & 12.94 & 4.0 & 0.0032 & 69.4 & 5.21 \\
\hline NGC $4772 .$. & 125329.10 & 021011.0 & 0.45 & 0.397 & 1.694 & 21.29 & 23.70 & 0.33 & 0.92 & 11.96 & 13.28 & 1.0 & 0.0032 & 69.4 & 5.21 \\
\hline NGC 5273 & 134208.40 & 353912.0 & 0.29 & 0.512 & 1.377 & 21.87 & 23.27 & 0.34 & 0.85 & 12.44 & 13.79 & -2.0 & 0.0037 & 80.3 & 6.02 \\
\hline NGC 5377.... & 135616.80 & 471417.0 & 1.00 & 0.561 & 1.858 & 23.29 & 25.14 & 0.37 & 0.90 & 12.24 & 13.66 & 1.0 & 0.0063 & 137. & 10.3 \\
\hline 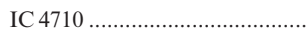 & 182838.20 & -665854.0 & 0.79 & 0.632 & 1.815 & 22.38 & 23.92 & -0.10 & 0.57 & 12.50 & 12.75 & 9.0 & 0.0021 & 45.6 & 3.42 \\
\hline NGC 7673 & 232741.60 & 233524.0 & 0.91 & 0.155 & 0.644 & 19.58 & 21.92 & -0.33 & 0.41 & 13.17 & 12.84 & 5.0 & 0.0119 & 258. & 19.4 \\
\hline
\end{tabular}

Note.- Unless stated otherwise, all table entries are taken or derived from the RC3 catalogue (de Vaucouleurs et al. 1991). Col. (1): Galaxy name. Cols. (2) and (3): J2000.0 coordinates of the galaxy centers as measured on the Digital Sky Survey (DSS). Units of right ascension are hours, minutes, and seconds, and units of declination are degrees, arcminutes, and arcseconds. In a few cases of complex galaxies, galaxy mergers, or galaxies in small groups, the listed coordinates refer to a "center-of-gravity position," allowing optimal centering inside the WFPC2 FOV. Col. (4): Ratio of the minor and major axis isophotal diameter at the $\mathrm{SB}(B)=25.0 \mathrm{mag} \operatorname{arcsec}^{-2}$ isophote. Col. (5): Half-light radius (units of $\operatorname{arcmin}$ ). Col. (6): Radius at the $\mathrm{SB}(B)=25.0 \mathrm{mag}$ arcsec ${ }^{-2}$ isophote (units of arcmin). Col. (7): Predicted average SB in F300W out to $r_{e}$ (units of $\mathrm{mag}_{\operatorname{arcsec}}^{-2}$; see $\S 2.2$ ). Col. (8): Predicted average SB in F300W out to $r_{25}$ (units of mag $\operatorname{arcsec}^{-2}$; see $\S 2.2$ ). Col. (9): Measured Johnson $(U-B)$ aperture color (mag). Col. (10): Measured ( $\left.B-V\right)$ aperture color (mag). Col. (11): Total $B$-band magnitude. Col. (12): Predicted total F300W magnitude (see $\S 2.2$ ). Col. (13): Revised galaxy T type (see also Fig. 2a). Col. (14): Galactocentric galaxy redshift, as derived from the RC3. Col. (15): Number of parsecs per arcsec at the distance of the galaxy, assuming a Hubble constant of $67 \mathrm{~km} \mathrm{~s}^{-1} \mathrm{Mpc}^{-1}$. Col. (16): Physical size (in kpc) corresponding to a single WFPC2 CCD of 75" $($ col. $[16]=$ col. $[15] \times 0.075)$. 


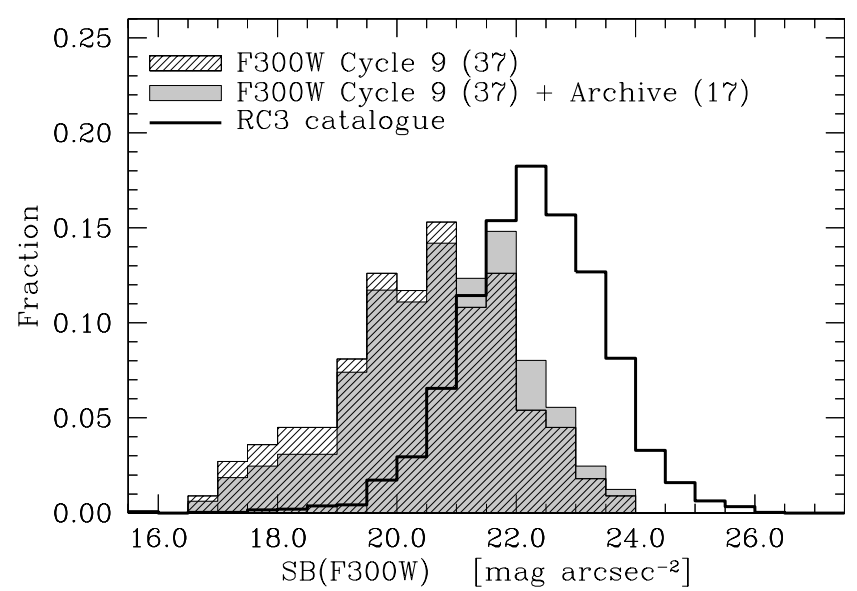

FIG. 1.-Normalized distribution of the predicted average mid-UV SB out to $r_{e}$ (see $\S 2.1 .2$ ) for the 37 galaxies observed during HST Cycle 9 (hashed histogram) and for the full galaxy sample (solid histogram), which includes 17 galaxies with mid-UV data taken prior to Cycle 9. For comparison, we also show the SB distribution for the 3009 galaxies in the RC3 with measured $B_{T},(U-B)$, and $r_{e}$ (open histogram). The galaxies were selected to have $\mu_{\mathrm{F} 300 \mathrm{~W}} \lesssim 23 \mathrm{mag}$ arcsec ${ }^{-2}$, allowing us to detect each object in F300W in no more than a single $H S T$ orbit. For our purpose of comparing nearby and distant galaxies this imposed SB bias is justified, since the strong cosmological SB dimming acts similarly in hiding lower SB objects at high redshifts from deep surveys.

"center-of-mass" position, allowing optimal centering inside the WFPC2 FOV. Column (4) shows the apparent axis ratio of the minor and major axis isophotal diameter measured at the $\mu_{B}=25.0 \mathrm{mag} \operatorname{arcsec}^{-2}$ isophote. The halflight radius, $r_{e}$, and the radius at the $\mu_{B}=25.0 \mathrm{mag} \mathrm{arcsec}-2$ isophote, $r_{25}$, are listed in columns (5) and (6), respectively. The predicted average SB in F300W out to $r_{e}$ and $r_{25}$ and the predicted total F300W magnitude (see $\S 2.2$ ) are tabulated in columns (7), (8), and (12). Column (13) lists the revised T type (numeric morphological type on the 16 step de Vaucouleurs scale). The redshifts in column (14) are given with respect to the galactic standard of rest. Column (15) lists for each galaxy the physical image scale in $\mathrm{pc}^{-\operatorname{arcsec}^{-1}}$ at the distance of the galaxy, assuming a Hubble constant $H_{0}=67$ $\mathrm{km} \mathrm{s}^{-1} \mathrm{Mpc}^{-1}$. Column (16) gives the physical size of a single WFC CCD frame at that redshift. For the typical redshift range, $z \simeq 0.004-0.02$ (i.e., Virgo/Coma Cluster-like distances), a single WFPC2 CCD covers $6.8-34 \mathrm{kpc}$.

The SB selection of the sample is illustrated in Figure 1, which shows the normalized distributions of the predicted average $\mu_{\mathrm{F} 300 \mathrm{w}}$ out to $r_{e}$ for the full (54 galaxy) sample and for the 37 galaxies in the Cycle 9 WFPC2 mid-UV sample, and compares these with the distribution over SB derived for the 3009 galaxies in the RC3 with measured $B_{T},(U-B)$, and $r_{e}$. Clearly, we sample predominantly galaxies with higher SB than the median SB value in the RC3, which is $\mu_{\mathrm{F} 300 \mathrm{~W}} \simeq 22.3 \mathrm{mag} \mathrm{arcsec}^{-2}$.

As nearby galaxy samples like the RC3 are also limited by significant SB selection (Disney 1976; Driver 1999), this thus means that our Cycle $9 H S T$ sample represents only the tip of the local galaxy SB distribution. Figure 1 shows that our sample covers only about the brightest $50 \%$ SB percentile seen in the RC3, while it overrepresents the highest SB galaxies seen in the RC3. If one wanted to use our sample to represent all galaxies in the $\mathrm{RC} 3$ with $\mu_{\mathrm{F} 300 \mathrm{~W}} \leq 23 \mathrm{mag}$ $\operatorname{arcsec}^{-2}$, one would have to apply weights to each of our galaxies in a given observed SB bin that could be derived from dividing the two distributions over SB in Figure 1. This will obviously not work for galaxies with $\mu_{\mathrm{F} 300 \mathrm{~W}} \geq 23$ mag $\operatorname{arcsec}^{-2}$, since almost no galaxies at average SB levels that faint were observed with $H S T$.

As aforementioned, this SB selection is justified when the primary goal is to construct a sample for comparison of nearby and high-redshift galaxies, since the strong cosmological SB dimming limits our view of the high-redshift universe in a similar way (or worse). For example, simulations by R. A. Windhorst, C. Conselice, \& L. Petro (2002, in preparation), who investigate how the galaxies in the present sample would appear to NGST under realistic observing conditions, show that at very high redshifts $(z \sim 7-15)$ only the highest SB mid-UV structures (with average rest-frame $\mu_{\mathrm{F} 300 \mathrm{~W}}$ out to $r_{e}$ brighter than 20-21 mag arcsec ${ }^{-2}$ ) will be visible to NGST.

Figures $2 a-2 d$ show the distributions of morphological type, of apparent axis ratio $b / a$, of the estimated average $(U-B)$ color within the effective radius, and of the effective radius $r_{e}$, within the selected sample. These figures will help the reader to evaluate the usefulness or the weight to apply to individual galaxies in the present sample when using these data for his or her particular purpose. We stress that the present sample is not formally complete and therefore is not suitable for studies that rely on complete statistics (i.e., studies that cover the complete local galaxy luminosity function or the complete range in local galaxy SB). Nonetheless, important trends are visible in Figures $2 a-2 d$ that will help the reader to make our sample useful for high-redshift comparisons. These trends are as follows:

1. Figure $2 a$ shows that our selected mid-UV galaxies overemphasize late types a bit more than the RC3. This was done on purpose, since late types are the dominant galaxy population at high redshifts (see Driver et al. 1995b).

2 . Figure $2 b$ shows that our selected mid-UV galaxies sample the $b / a$ distribution of the RC3 fairly, given the small number statistics. Since Odewahn, Burstein, \& Windhorst (1997) found no significant trend in the galaxy $b / a$ distribution from the RC3 level ( $B \lesssim 15 \mathrm{mag}$ ) all the way down to the HDF limit ( $B \lesssim 28 \mathrm{mag}$ ), our nearby comparison sample is thus a fair one in terms of galaxy ellipticities at all redshifts.

3. Figure $2 c$ shows that our selected mid-UV galaxies overemphasize the bluest object in $(U-B)$ a bit more than the RC3. Like Figure $2 a$, this was done on purpose, since faint blue galaxies are the dominant population at faint magnitudes.

4. Figure $2 d$ shows that our selected mid-UV galaxies overemphasize the intrinsically smaller galaxies in $r_{e}$ more than the RC3, which was again done on purpose, since faint blue galaxies are on average significantly smaller than the ones seen nearby (see Odewahn et al. 1996; Pascarelle et al. 1996; Cohen et al. 2002).

Again, any nonrepresentativeness of our galaxy sample compared to the RC3, or to any other galaxy catalog for that matter, can be addressed by applying the appropriate weights to individual galaxies in our sample when comparing it to any other samples. These weights can be determined from the ratios between the observed distributions in Figures $2 a-2 d$ or between our sample and any sample other than RC3. 

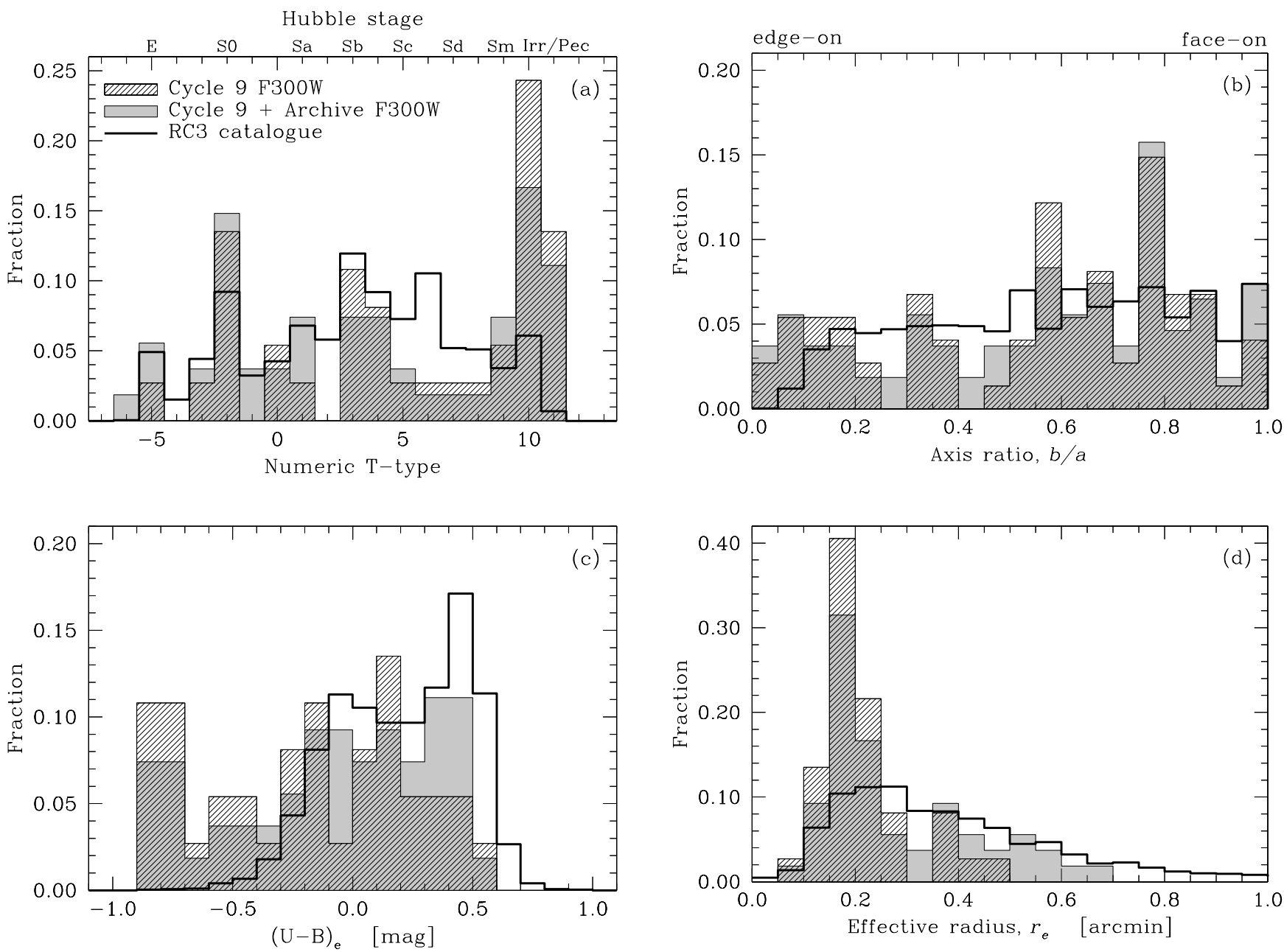

FIG. 2.-Other properties of the selected galaxy sample. As in Fig. 1, we present normalized distributions for the Cycle 9 galaxies (hashed), the full sample (solid), and the RC3 (open) of (a) morphological type, $(b)$ apparent axis ratio $b / a,(c)$ average $(U-B)$ color out to $r_{e}$, and $(d)$ effective radius $r_{e}$. Taking deviations due to small number statistics into account, our sample approximates the RC3 distribution, except that for the purpose of comparison with high-redshift objects, we placed extra emphasis on the very latest types, bluest optical galaxy colors, and smallest angular sizes (to fit the FOV of the WFPC2). We also somewhat overrepresent highly inclined systems.

\subsection{Observations}

\subsubsection{HST/WFPC2 Observations}

All 37 selected galaxies were observed with WFPC2 during Cycle 9. Typically, we exposed $2 \times(800-1000) \mathrm{s}$ in F300W and $2 \times(100-160) \mathrm{s}$ in F814W. Exposures times were made flexible to optimally use the full HST orbit allocated per galaxy. Of the 24 galaxies located in the CVZ, 11 could indeed be scheduled there during Cycle 9. For these, exposure times were $3 \times(400-500) \mathrm{s}$ in both the F255W and F300W filters and $2 \times(100-160) \mathrm{s}$ in F814W. In none of the 37 Cycle 9 targets did we see evidence for elevated sky levels in F300W (see $\S 2.1 .4$ ).

We adopted a linear dither pattern such that exposures in the same filter are offset by $n+0.34$ WFC pixels in the case of two exposures per filter (non-CVZ) and $n+0.33$ and $n+0.66$ WFC pixels in the case of three exposures per filter (CVZ). Here $n$ is a multiple of 5 pixels (ideally 10-30 pixels in both directions to fill in the vignetted regions between the WFPC2 CCDs), and the pixel fractions assure optimal drizzling in a later stage. The actual shifts may slightly deviate from the listed values depending on the quality of the FGS lock achieved for a particular galaxy.

\subsubsection{Ground-based Observations}

Most of the ground-based UBVRI CCD images for the 37 galaxies in the present sample were obtained with the $1.8 \mathrm{~m}$ Vatican Advanced Technology Telescope (VATT) on Mount Graham, with additional images taken with the 0.9 $\mathrm{m}$ telescope at the Cerro Tololo Inter-American Observatory (CTIO), the Danish $1.5 \mathrm{~m}$ telescope at the European Southern Observatory (ESO), the $2.1 \mathrm{~m}$ telescope at the Kitt Peak National Observatory (KPNO), the $2.2 \mathrm{~m}$ University of Hawaii telescope at Mauna Kea (UH), or the $1.0 \mathrm{~m}$ Jacobus Kapteyn Telescope (JKT) at La Palma. The optical data were obtained mostly with $2 \mathrm{k} \times 2 \mathrm{k}$ CCDs. Further details are given in Matthews \& Gallagher (1997), Eskridge et al. (2002), and V. A. Taylor et al. (2003, in preparation).

Ground-based near-IR imaging was done using the CTIO $1.5 \mathrm{~m}$ telescope, the ESO $2.2 \mathrm{~m}$ telescope, the Carnegie $2.5 \mathrm{~m}$ du Pont telescope at Las Campanas (LCO), or the $3.8 \mathrm{~m}$ United Kingdom Infrared Telescope (UKIRT) at Mauna Kea, mostly with $256^{2} \mathrm{HgCdTe}$ arrays (and $1024^{2}$ in the case of the du Pont telescope). Images were obtained in $J H K$ or a subset of these filters. For further details on these near-IR 
data we refer the reader to de Grijs et al. (1997), de Jong \& van der Kruit (1994), and Eskridge et al. (2002).

The $J H K$ filters sample the underlying cooler (and usually older) stellar population, which in general also dominates the $R$ and $I$ bands, so that the RIJHK filters in general reveal similar galaxy morphology. Morphological contrast is added through the younger stellar populations in the bluer and UV filters. Since the older stellar population dominates the RIJHK fluxes of earlier type galaxies, a comparison of these filters can in principle provide a measure of the dust content, whose effects are strongly wavelength dependent, noting the usual caveat that effects from dust and metallicity are hard to separate and require spatially resolved spectroscopy in addition to broadband photometry to address this quantitatively.

We aimed for a photometric zero-point accuracy better than $0.03-0.05 \mathrm{mag}$, in the optical as well as the near-IR. The ground-based CCD images were reduced following standard procedures, as described in Matthews \& Gallagher (1997) and Neuschaefer \& Windhorst (1995).

In Table 2 we summarize our Cycle $9 H S T$ observations together with the UIT and ground-based images available to date. For WFPC2 observations, we list the HST roll angle (PA_V3) and the nominal FWHM of the HST pointspread function (PSF); for ground-based observations, we list the air mass and the median FWHM in each image stack. These FWHM values were measured from unsaturated stellar images using SExtractor (Bertin \& Arnouts 1996). We clipped the FWHM distribution at 0".3 to filter out cosmic rays and bad pixels and at $5^{\prime \prime}$ to filter out misclassified extended objects (e.g., H II or star-forming regions inside the galaxies of interest). These values, as well as the FWHM values of the Moffat (1969) profiles as fitted using the task imexamine within IRAF, are consistent to within $2.5 \%$ with a dispersion of $9 \%$. FWHM values could be measured for 124 (out of 131) ground-based images. For the remaining images that did not contain any unsaturated stars, the FWHM was estimated from the images taken closest in time that did. The mean (median) seeing over all

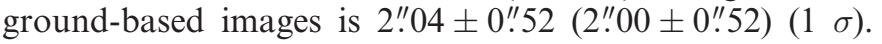
This rather large average image FWHM is due to the majority of VATT images having a focus component in their PSF as a result of the rapid change of focus with temperature in this $\mathrm{f} / 1$ telescope (for details see V. A. Taylor et al. 2003, in preparation). Even with FWHM $\sim 2$ ".0, these ground-based images are quite suitable for our current purpose of galaxy surface photometry in $U B V R$.

\subsubsection{Archival HST Images in Additional Filters}

Several of the galaxies in the present sample have been observed with HST in filters other than F300W and F255W or with instruments other than WFPC2. Maoz et al. (1996), for example, imaged the central $22^{\prime \prime} \times 22^{\prime \prime}$ in 110 nearby galaxies in the mid-UV (2300 $\AA$ ) using the Faint Object Camera (FOC). The red leak in the FOC mid-UV filters, however, is more severe ( 24\%; Keel \& Windhorst 1993) than that in the WFPC2 filters $(\simeq 5 \%-7 \%$; see $\S 2.4 .3)$, and the FOV is much smaller. Sixteen of our objects have been observed in other WFPC2 filters before, generally in a heterogeneous mixture of filters: F170W (far-UV), F336W, F439W, F555W, F606W, F702W, and/or some additional F814W images. These data are summarized in Table 3. In the archival WFPC2 exposures, the galaxy nucleus was often placed in the Planetary Camera (PC), which, as a result of its smaller pixel size, is less suited for accurate surface photometry. Furthermore, for galaxies $\sim 1^{\prime}$ in extent, more of a galaxy tends to be lost outside WFPC2's FOV when its nucleus is placed in the PC. We will study these archival WFPC2 images in the other filters, where appropriate, in future papers.

Where archival NICMOS images in the F110M/F110W, F160W, or F222M filters are available (GTO 7218, PI G. H. Rieke; see, e.g., Alonso-Herrero et al. 2000; GTO 7219, PI N. Z. Scoville; see Scoville et al. 2000; GO 7268, PI R. P. van der Marel; see Ravindranath et al. 2001; SNAP 7328, PI M. Malkan; see Quillen et al. 2001; SNAP 7330, PI J. Mulchaey; see Regan \& Mulchaey 1999), we use them in lieu of ground-based JHK data in Table 2 and Figure 4.

\subsection{Calibration and Processing of the HST Observations}

\subsubsection{Standard Processing}

Standard WFPC2 CCD processing following Windhorst et al. (1994b, 1994c), Windhorst, Keel, \& Pascarelle (1998a), Driver et al. (1995b), Neuschaefer \& Windhorst (1995), and Odewahn et al. (1996) included bias and dark subtraction, as well as flat-fielding. Photometric calibration was done using the STSDAS on-the-fly-reduction (OTFR) routines as available since summer 2000. Custom calibration, in general, does not significantly improve upon the STScI WFPC2 pipeline, owing to the significant work that went into building and improving that pipeline. The OTFR takes into account the latest improvements in knowledge of the instrument every time one retrieves data from the HST Archive.

Because the mid-UV images have extremely low sky background levels, the background subtraction is limited by the quality of the bias and dark current removal. It is therefore important that the very best possible biases and latest dark current and hot pixel maps are used. We paid close attention to whether the correct dark frames were used when observations were taken near the monthly warm-up of WFPC2 (to decontaminate the optics and anneal many of the new hot pixels). The OTFR uses the best available superdark taken after the relevant science images but before the next decontamination. Hence, it is conservative in nature, repairing more pixels than needed, but never too few. We reran the OTFR on all data 1 month after the last images for this project were taken (in 2001 April) to incorporate the latest knowledge on the WFPC2 data. The difference between this second run and the first was very small but went in the direction of removing a more appropriate (smaller) number of pixels deemed "hot."

We co-added all images in the same filter after registration using integer pixel shifts. Our in-house IDL routine STCombine (Pascarelle, Windhorst, \& Keel 1998; Cohen et al. 2002) was used to optimally remove the signal induced by the many cosmic-ray hits. This routine, optimized for the low-signal domain, applies a one-sided $2 \sigma$ rejection in creating the final stacked image (following Windhorst, Franklin, \& Neuschaefer 1994a).

\subsubsection{Achieved $S / N$ in the WFPC2 Images}

The zodiacal sky background at the north ecliptic pole is $\sim 24.0 \mathrm{mag} \operatorname{arcsec}^{-2}$ in F300W (Windhorst et al. 1994b, 1998a) and $\sim 24.7 \mathrm{mag} \operatorname{arcsec}^{-2}$ in F255W (Cornett et al. 1994) and is as low in the sunlit part of the orbit as it is in the occulted part (unless the angle to the Earth's limb becomes 
TABLE 2

Summary of $H S T$, UIT, and Ground-Based Images of Our Cycle 9 Sample

\begin{tabular}{|c|c|c|c|c|c|c|c|c|c|}
\hline $\begin{array}{l}\text { Object } \\
\text { (1) }\end{array}$ & $\begin{array}{c}\text { Telescope } \\
\text { (2) }\end{array}$ & $\begin{array}{l}\text { Filter } \\
\text { (3) }\end{array}$ & $\begin{array}{l}\text { Exposure Time } \\
\text { (s) } \\
\text { (4) }\end{array}$ & $\begin{array}{c}\text { Air Mass } \\
\text { (5) }\end{array}$ & $\begin{array}{l}\text { Orientation } \\
\text { (deg) } \\
\text { (6) }\end{array}$ & $\begin{array}{l}\text { Zero Point } \\
\text { (mag) } \\
(7)\end{array}$ & $\begin{array}{c}\text { Plate Scale } \\
\left(\operatorname{arcsec} \text { pixel }^{-1}\right) \\
(8)\end{array}$ & $\begin{array}{c}\text { FWHM } \\
(\operatorname{arcsec}) \\
(9)\end{array}$ & $\begin{array}{c}\text { Figure } \\
(10)\end{array}$ \\
\hline \multirow[t]{6}{*}{ NGC 1140 (Mrk 1063).. } & $H S T$ & F300W & $2 \times 900.0$ & 0 & 71.933 & 19.433 & 0.0996 & 0.04 & $3 / 4.28$ \\
\hline & $H S T$ & F814W & $2 \times 100.0$ & 0 & 71.933 & 21.659 & 0.0996 & 0.08 & $3 / 4.28$ \\
\hline & VATT & $U$ & $1 \times 300.0$ & 1.364 & 0.0 & $\ldots$ & 0.3746 & 2.15 & 4.28 \\
\hline & VATT & $B$ & $1 \times 180.0$ & 1.360 & 0.0 & $\ldots$ & 0.3746 & 2.59 & 4.28 \\
\hline & VATT & $V$ & $1 \times 180.0$ & 1.358 & 0.0 & $\ldots$ & 0.3746 & 2.49 & 4.28 \\
\hline & POSS II & IIIaF & $1 \times 3000.0$ & & 0.0 & $\ldots$ & 1.00 & 3.86 & 4.28 \\
\hline \multirow{6}{*}{ ESO 418-G008 } & $H S T$ & F300W & $2 \times 900.0$ & 0 & 151.410 & 19.433 & 0.0996 & 0.04 & $3 / 4.25$ \\
\hline & $H S T$ & F814W & $2 \times 130.0$ & 0 & 151.410 & 21.659 & 0.0996 & 0.08 & $3 / 4.25$ \\
\hline & VATT & $U$ & $1 \times 300.0$ & 2.184 & 0.0 & $\ldots$ & 0.3746 & 2.80 & 4.25 \\
\hline & VATT & $B$ & $1 \times 360.0$ & 2.186 & 0.0 & $\ldots$ & 0.3746 & 2.76 & 4.25 \\
\hline & VATT & $V$ & $1 \times 240.0$ & 2.191 & 0.0 & $\ldots$ & 0.3746 & 2.50 & 4.25 \\
\hline & VATT & $R$ & $1 \times 120.0$ & 2.197 & 0.0 & $\ldots$ & 0.3746 & 2.53 & 4.25 \\
\hline \multirow[t]{6}{*}{ 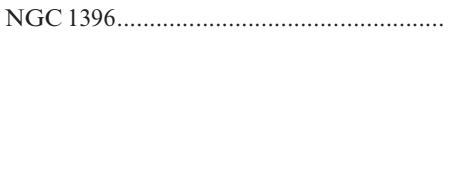 } & UIT & $150 \mathrm{~nm}$ & $1 \times 1454.5$ & 0 & 83.832 & 16.928 & 1.200 & 3.03 & 4.02 \\
\hline & UIT & $250 \mathrm{~nm}$ & $1 \times 1099.5$ & 0 & 83.831 & 18.658 & 1.200 & 2.71 & 4.02 \\
\hline & $H S T$ & F300W & $2 \times 900.0$ & 0 & 22.975 & 19.433 & 0.0996 & 0.04 & $3 / 4.02$ \\
\hline & $H S T$ & F814W & $2 \times 130.0$ & 0 & 22.975 & 21.659 & 0.0996 & 0.08 & $3 / 4.02$ \\
\hline & POSS I & IIIaJ & $1 \times 3600.0$ & $\ldots$ & 0.0 & $\ldots$ & 1.70 & 5.0 & 4.02 \\
\hline & POSS II & IIIaF & $1 \times 3600.0$ & $\ldots$ & 0.0 & $\ldots$ & 1.00 & 3.56 & 4.02 \\
\hline \multirow[t]{5}{*}{ NGC $1510 .}$. & UIT & $150 \mathrm{~nm}$ & $1 \times 948.5$ & 0 & 0.020 & 16.806 & 1.137 & 3.03 & 4.26 \\
\hline & $H S T$ & F300W & $2 \times 900.0$ & 0 & 13.650 & 19.433 & 0.0996 & 0.04 & $3 / 4.26$ \\
\hline & $H S T$ & F814W & $2 \times 160.0$ & 0 & 13.650 & 21.659 & 0.0996 & 0.08 & $3 / 4.26$ \\
\hline & POSS I & IIIaJ & $1 \times 4200.0$ & $\ldots$ & 0.0 & $\ldots$ & 1.70 & 4.8 & 4.26 \\
\hline & POSS II & IIIaF & $1 \times 3900.0$ & $\ldots$ & 0.0 & $\ldots$ & 1.00 & 3.52 & 4.26 \\
\hline \multirow[t]{5}{*}{ 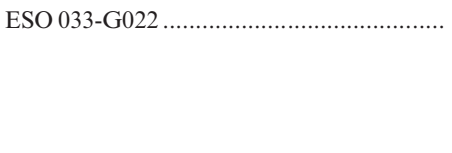 } & $H S T$ & F300W & $2 \times 1000.0$ & 0 & 254.257 & 19.433 & 0.0996 & 0.04 & $3 / 4.19$ \\
\hline & $H S T$ & F814W & $2 \times 130.0$ & 0 & 254.257 & 21.659 & 0.0996 & 0.08 & $3 / 4.19$ \\
\hline & ESO $1.5 \mathrm{~m}$ & B & $2 \times 1500.0$ & $\ldots$ & 0.28 & 22.331 & 0.36 & 1.7 & 4.19 \\
\hline & ESO $1.5 \mathrm{~m}$ & $V$ & $1 \times 2100.0$ & $\ldots$ & 0.28 & 23.058 & 0.36 & 1.6 & 4.19 \\
\hline & ESO $1.5 \mathrm{~m}$ & $I$ & $2 \times 900.0$ & $\ldots$ & 0.28 & 22.351 & 0.36 & 0.9 & 4.19 \\
\hline \multirow[t]{8}{*}{ 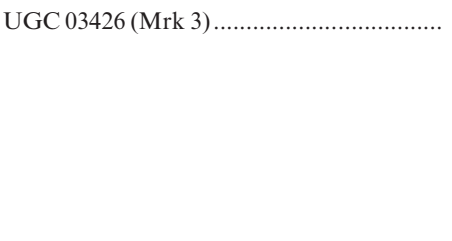 } & UIT & $150 \mathrm{~nm}$ & $2 \times 1261.0$ & 0 & 327.300 & 16.806 & 1.137 & 3.03 & 4.05 \\
\hline & $H S T$ & F255W & $3 \times 466.7$ & 0 & 119.723 & 17.037 & 0.0996 & 0.04 & $3 / 4.05$ \\
\hline & $H S T$ & F300W & $3 \times 500.0$ & 0 & 119.723 & 19.433 & 0.0996 & 0.04 & $3 / 4.05$ \\
\hline & $H S T$ & F814W & $2 \times 130.0$ & 0 & 119.723 & 21.659 & 0.0996 & 0.08 & $3 / 4.05$ \\
\hline & VATT & $U$ & $1 \times 300.0$ & 1.462 & 0.0 & $\ldots$ & 0.3746 & 1.96 & 4.05 \\
\hline & VATT & $B$ & $1 \times 180.0$ & 1.502 & 0.0 & $\ldots$ & 0.3746 & 1.62 & 4.05 \\
\hline & VATT & $V$ & $1 \times 120.0$ & 1.522 & 0.0 & $\ldots$ & 0.3746 & 1.52 & 4.05 \\
\hline & VATT & $R$ & $1 \times 30.0$ & 1.553 & 0.0 & $\ldots$ & 0.3746 & 1.42 & 4.05 \\
\hline \multirow[t]{7}{*}{ 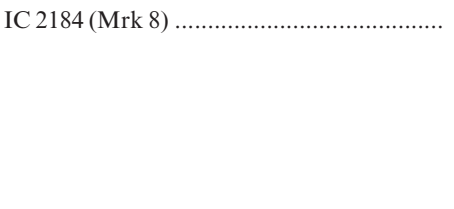 } & $H S T$ & F255W & $3 \times 466.7$ & 0 & 139.416 & 17.037 & 0.0996 & 0.04 & $3 / 4.37$ \\
\hline & $H S T$ & F300W & $3 \times 500.0$ & 0 & 139.416 & 19.433 & 0.0996 & 0.04 & $3 / 4.37$ \\
\hline & $H S T$ & F814W & $2 \times 130.0$ & 0 & 139.416 & 21.659 & 0.0996 & 0.08 & $3 / 4.37$ \\
\hline & VATT & $U$ & $1 \times 600.0$ & 1.298 & 0.0 & $\ldots$ & 0.3746 & 2.12 & 4.37 \\
\hline & VATT & $B$ & $1 \times 300.0$ & 1.298 & 0.0 & $\ldots$ & 0.3746 & 1.96 & 4.37 \\
\hline & VATT & $V$ & $1 \times 240.0$ & 1.298 & 0.0 & $\ldots$ & 0.3746 & 2.00 & 4.37 \\
\hline & VATT & $R$ & $1 \times 180.0$ & 1.302 & 0.0 & $\ldots$ & 0.3746 & 1.70 & 4.37 \\
\hline 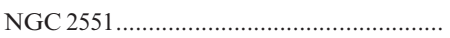 & UIT & $150 \mathrm{~nm}$ & $1 \times 454.5$ & 0 & 43.306 & 16.928 & 1.200 & 3.03 & 4.10 \\
\hline & UIT & $250 \mathrm{~nm}$ & $1 \times 454.5$ & 0 & 43.307 & 18.658 & 1.200 & 2.71 & 4.10 \\
\hline & $H S T$ & F300W & $2 \times 1000.0$ & 0 & 232.495 & 19.433 & 0.0996 & 0.04 & $3 / 4.10$ \\
\hline & $H S T$ & F814W & $2 \times 130.0$ & 0 & 232.495 & 21.659 & 0.0996 & 0.08 & $3 / 4.10$ \\
\hline & VATT & $U$ & $1 \times 600.0$ & 1.325 & 0.0 & $\ldots$ & 0.3746 & 1.60 & 4.10 \\
\hline & VATT & $B$ & $1 \times 180.0$ & 1.325 & 0.0 & $\ldots$ & 0.3746 & 1.24 & 4.10 \\
\hline & VATT & $V$ & $1 \times 120.0$ & 1.324 & 0.0 & $\ldots$ & 0.3746 & 1.15 & 4.10 \\
\hline & VATT & $R$ & $1 \times 120.0$ & 1.324 & 0.0 & $\ldots$ & 0.3746 & 1.00 & 4.10 \\
\hline UGC 05028/29 (Arp 300). & $H S T$ & F300W & $2 \times 950.0$ & 0 & 350.000 & 19.433 & 0.0996 & 0.04 & $3 / 4.23$ \\
\hline & $H S T$ & F814W & $2 \times 160.0$ & 0 & 350.000 & 21.659 & 0.0996 & 0.08 & $3 / 4.23$ \\
\hline & POSS II & IIIaJ & $1 \times 4800.0$ & $\ldots$ & 0.0 & $\ldots$ & 1.00 & 3.48 & 4.23 \\
\hline & POSS II & IIIaF & $1 \times 3000.0$ & $\ldots$ & 0.0 & $\ldots$ & 1.00 & 3.40 & 4.23 \\
\hline UGC $05101 \ldots \ldots .$. & $H S T$ & F255W & $3 \times 466.7$ & 0 & 165.068 & 17.037 & 0.0996 & 0.04 & $3 / 4.08$ \\
\hline & $H S T$ & F300W & $3 \times 500.0$ & 0 & 165.068 & 19.433 & 0.0996 & 0.04 & $3 / 4.08$ \\
\hline & $H S T$ & F814W & $2 \times 130.0$ & 0 & 165.068 & 21.659 & 0.0996 & 0.08 & $3 / 4.08$ \\
\hline & VATT & $U$ & $4 \times 300.0$ & 1.223 & 0.0 & 25.000 & 0.3746 & 1.9 & 4.08 \\
\hline & VATT & $B$ & $1 \times 300.0$ & 1.203 & 0.0 & 25.000 & 0.3746 & 2.0 & 4.08 \\
\hline & VATT & $V$ & $1 \times 240.0$ & 1.197 & 0.0 & 25.000 & 0.3746 & 2.1 & 4.08 \\
\hline & VATT & $R$ & $1 \times 180.0$ & 1.192 & 0.0 & 25.000 & 0.3746 & 2.1 & 4.08 \\
\hline UGC $05189 \ldots \ldots$ & $H S T$ & F300W & $2 \times 900.0$ & 0 & 230.001 & 19.433 & 0.0996 & 0.04 & $3 / 4.33$ \\
\hline & $H S T$ & F814W & $2 \times 100.0$ & 0 & 230.001 & 21.659 & 0.0996 & 0.08 & $3 / 4.33$ \\
\hline & VATT & $U$ & $2 \times 1200.0$ & 1.1 & 0.0 & 25.000 & 0.3746 & 1.8 & 4.33 \\
\hline & VATT & $B$ & $2 \times 600.0$ & 1.1 & 0.0 & 25.000 & 0.3746 & 1.6 & 4.33 \\
\hline & VATT & $V$ & $4 \times 480.0$ & 1.1 & 0.0 & 25.000 & 0.3746 & 1.6 & 4.33 \\
\hline & VATT & $R$ & $2 \times 360.0$ & 1.1 & 0.0 & 25.000 & 0.3746 & 1.5 & 4.33 \\
\hline 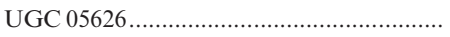 & $H S T$ & F255W & $3 \times 466.7$ & 0 & 170.844 & 17.037 & 0.0996 & 0.04 & $3 / 4.29$ \\
\hline & $H S T$ & F300W & $3 \times 500.0$ & 0 & 170.844 & 19.433 & 0.0996 & 0.04 & $3 / 4.29$ \\
\hline & $H S T$ & F814W & $2 \times 130.0$ & 0 & 170.844 & 21.659 & 0.0996 & 0.08 & $3 / 4.29$ \\
\hline & VATT & $U$ & $2 \times 1200.0$ & 1.417 & 0.0 & 25.000 & 0.3746 & 2.0 & 4.29 \\
\hline & VATT & $B$ & $2 \times 600.0$ & 1.427 & 0.0 & 25.000 & 0.3746 & 1.9 & 4.29 \\
\hline
\end{tabular}


TABLE 2-Continued

\begin{tabular}{|c|c|c|c|c|c|c|c|c|c|}
\hline $\begin{array}{l}\text { Object } \\
\text { (1) }\end{array}$ & $\begin{array}{l}\text { Telescope } \\
\text { (2) }\end{array}$ & $\begin{array}{l}\text { Filter } \\
\text { (3) }\end{array}$ & $\begin{array}{c}\text { Exposure Time } \\
\text { (s) } \\
\text { (4) }\end{array}$ & $\begin{array}{l}\text { Air Mass } \\
\text { (5) }\end{array}$ & $\begin{array}{l}\text { Orientation } \\
\text { (deg) } \\
\text { (6) }\end{array}$ & $\begin{array}{l}\text { Zero Point } \\
\quad(\mathrm{mag}) \\
(7)\end{array}$ & $\begin{array}{c}\text { Plate Scale } \\
\left(\operatorname{arcsec}^{-1 x x^{-1}}\right) \\
\text { (8) }\end{array}$ & $\begin{array}{l}\text { FWHM } \\
(\operatorname{arcsec}) \\
(9)\end{array}$ & $\begin{array}{l}\text { Figure } \\
(10)\end{array}$ \\
\hline & VATT & $V$ & $2 \times 480.0$ & 1.436 & 0.0 & 25.000 & 0.3746 & 1.8 & 4.29 \\
\hline & VATT & $R$ & $2 \times 360.0$ & 1.444 & 0.0 & 25.000 & 0.3746 & 1.8 & 4.29 \\
\hline \multirow{8}{*}{ NGC 3310 (Arp 217) ………...................... } & UIT & $150 \mathrm{~nm}$ & $1 \times 1130.5$ & 0 & 112.049 & 16.806 & 1.1367 & 3.03 & 4.13 \\
\hline & $H S T$ & F300W & $2 \times 950.0$ & 0 & 165.015 & 19.433 & 0.0996 & 0.04 & $3 / 4.13$ \\
\hline & $H S T$ & F814W & $2 \times 130.0$ & 0 & 165.015 & 21.659 & 0.0996 & 0.08 & $3 / 4.13$ \\
\hline & VATT & $U$ & $1 \times 300.0$ & 1.225 & 0.0 & & 0.3746 & 2.10 & 4.13 \\
\hline & VATT & $B$ & $1 \times 150.0$ & 1.232 & 0.0 & $\ldots$ & 0.3746 & 1.62 & 4.13 \\
\hline & VATT & $V$ & $1 \times 150.0$ & 1.242 & 0.0 & $\ldots$ & 0.3746 & 2.21 & 4.13 \\
\hline & VATT & $R$ & $1 \times 150.0$ & 1.251 & 0.0 & & 0.3746 & 1.37 & 4.13 \\
\hline & $H S T$ & F160W & $1 \times 256.0$ & 0 & 80.830 & 21.100 & 0.2000 & 0.20 & 4.13 \\
\hline \multirow{7}{*}{ MCG +06-24-047 ......................................... } & UIT & $150 \mathrm{~nm}$ & $1 \times 1430.5$ & 0 & 127.736 & 16.806 & 1.1367 & 3.03 & 4.22 \\
\hline & $H S T$ & F300W & $2 \times 900.0$ & 0 & 145.410 & 19.433 & 0.0996 & 0.04 & $3 / 4.22$ \\
\hline & $H S T$ & F814W & $2 \times 130.0$ & 0 & 145.410 & 21.659 & 0.0996 & 0.08 & $3 / 4.22$ \\
\hline & VATT & $U$ & $2 \times 600.0$ & 1.032 & 0.0 & 25.000 & 0.3746 & 2.5 & 4.22 \\
\hline & VATT & $B$ & $2 \times 300.0$ & 1.030 & 0.0 & 25.000 & 0.3746 & 2.8 & 4.22 \\
\hline & VATT & $V$ & $2 \times 240.0$ & 1.029 & 0.0 & 25.000 & 0.3746 & 2.5 & 4.22 \\
\hline & VATT & $R$ & $2 \times 180.0$ & 1.186 & 0.0 & 25.000 & 0.3746 & 2.5 & 4.22 \\
\hline \multirow[t]{8}{*}{ 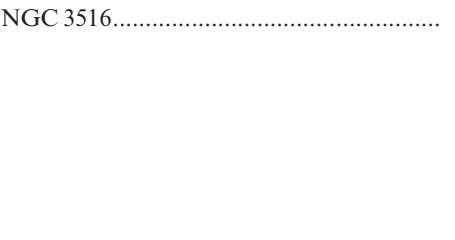 } & UIT & $150 \mathrm{~nm}$ & $1 \times 1480.5$ & 0 & 299.210 & 16.806 & 1.1367 & 3.03 & 4.04 \\
\hline & $H S T$ & $\mathrm{~F} 255 \mathrm{~W}$ & $3 \times 466.7$ & 0 & 256.848 & 17.037 & 0.0996 & 0.04 & $3 / 4.04$ \\
\hline & $H S T$ & F300W & $3 \times 500.0$ & 0 & 256.848 & 19.433 & 0.0996 & 0.04 & $3 / 4.04$ \\
\hline & $H S T$ & F814W & $2 \times 130.0$ & 0 & 256.848 & 21.659 & 0.0996 & 0.08 & $3 / 4.04$ \\
\hline & VATT & $U$ & $1 \times 300.0$ & 1.636 & 0.0 & $\ldots$ & 0.3746 & 1.85 & 4.04 \\
\hline & VATT & $B$ & $1 \times 150.0$ & 1.655 & 0.0 & & 0.3746 & 1.80 & 4.04 \\
\hline & VATT & $V$ & $1 \times 100.0$ & 1.662 & 0.0 & $\ldots$ & 0.3746 & 1.66 & 4.04 \\
\hline & VATT & $R$ & $1 \times 180.0$ & 1.711 & 0.0 & $\ldots$ & 0.3746 & 2.00 & 4.04 \\
\hline \multirow[t]{8}{*}{ NGC 3690/IC 0694 (Arp 299, Mrk 171)..... } & $H S T$ & F255W & $3 \times 466.7$ & 0 & 257.350 & 17.037 & 0.0996 & 0.04 & $3 / 4.34$ \\
\hline & $H S T$ & F300W & $3 \times 500.0$ & 0 & 257.350 & 19.433 & 0.0996 & 0.04 & $3 / 4.34$ \\
\hline & $H S T$ & F814W & $2 \times 130.0$ & 0 & 257.350 & 21.659 & 0.0996 & 0.08 & $3 / 4.34$ \\
\hline & VATT & $U$ & $2 \times 600.0$ & 1.120 & 0.0 & $\ldots$ & 0.3746 & 2.84 & 4.34 \\
\hline & $\mathrm{UH} 2.2 \mathrm{~m}$ & $B$ & $1 \times 600.0$ & 1.283 & 359.910 & $\ldots$ & 0.4400 & 1.4 & 4.34 \\
\hline & $\mathrm{UH} 2.2 \mathrm{~m}$ & $V$ & $1 \times 900.0$ & 1.284 & 359.910 & $\ldots$ & 0.4400 & 1.5 & 4.34 \\
\hline & $\mathrm{UH} 2.2 \mathrm{~m}$ & $R$ & $1 \times 600.0$ & 1.283 & 359.910 & $\ldots$ & 0.4400 & 1.5 & 4.34 \\
\hline & $\mathrm{UH} 2.2 \mathrm{~m}$ & $K$ & $5 \times 120.0$ & 1.286 & 0.006 & & 0.2200 & 1.1 & 4.34 \\
\hline \multirow{7}{*}{ UGC 06527 (Arp 322, Mrk 176) .................. } & $H S T$ & F300W & $2 \times 950.0$ & 0 & 251.824 & 19.433 & 0.0996 & 0.04 & $3 / 4.35$ \\
\hline & $H S T$ & F814W & $2 \times 130.0$ & 0 & 251.824 & 21.659 & 0.0996 & 0.08 & $3 / 4.35$ \\
\hline & VATT & $U$ & $2 \times 1200.0$ & 1.083 & 0.0 & 25.000 & 0.3746 & 2.3 & 4.35 \\
\hline & VATT & $B$ & $2 \times 600.0$ & 1.078 & 0.0 & 25.000 & 0.3746 & 2.6 & 4.35 \\
\hline & VATT & $V$ & $2 \times 480.0$ & 1.077 & 0.0 & 25.000 & 0.3746 & 2.4 & 4.35 \\
\hline & VATT & $R$ & $2 \times 360.0$ & 1.212 & 0.0 & 25.000 & 0.3746 & 2.5 & 4.35 \\
\hline & $H S T$ & F160W & $1 \times 256.0$ & 0 & -6.017 & 21.100 & 0.2000 & 0.20 & 4.35 \\
\hline \multirow[t]{8}{*}{ UGC $06697 .}$. & UIT & $150 \mathrm{~nm}$ & $1 \times 529.5$ & 0 & 84.124 & 16.928 & 1.200 & 3.03 & 4.17 \\
\hline & UIT & $250 \mathrm{~nm}$ & $1 \times 105.5$ & 0 & 84.125 & 18.658 & 1.200 & 2.71 & 4.17 \\
\hline & $H S T$ & F300W & $2 \times 900.0$ & 0 & 286.763 & 19.433 & 0.0996 & 0.04 & $3 / 4.17$ \\
\hline & $H S T$ & F814W & $2 \times 100.0$ & 0 & 286.763 & 21.659 & 0.0996 & 0.08 & $3 / 4.17$ \\
\hline & VATT & $U$ & $2 \times 600.0$ & 1.035 & 0.0 & 25.000 & 0.3746 & 2.3 & 4.17 \\
\hline & VATT & $B$ & $2 \times 300.0$ & 1.032 & 0.0 & 25.000 & 0.3746 & 2.4 & 4.17 \\
\hline & VATT & $V$ & $3 \times 240.0$ & 1.043 & 0.0 & 25.000 & 0.3746 & 2.2 & 4.17 \\
\hline & VATT & $R$ & $2 \times 180.0$ & 1.162 & 0.0 & 25.000 & 0.3746 & 2.6 & 4.17 \\
\hline CGCG 097-094 (observed in the field of & & & & & & & & & \\
\hline MCG +03-30-071) …………............ & UIT & $150 \mathrm{~nm}$ & $1 \times 529.5$ & 0 & 84.124 & 16.928 & 1.200 & 3.03 & 4.01 \\
\hline & UIT & $250 \mathrm{~nm}$ & $1 \times 105.5$ & 0 & 84.125 & 18.658 & 1.200 & 2.71 & 4.01 \\
\hline & $H S T$ & F300W & $2 \times 900.0$ & 0 & 300.002 & 19.433 & 0.0996 & 0.04 & $3 / 4.01$ \\
\hline & $H S T$ & F814W & $2 \times 100.0$ & 0 & 300.002 & 21.659 & 0.0996 & 0.08 & $3 / 4.01$ \\
\hline & VATT & $U$ & $2 \times 600.0$ & 1.045 & 0.0 & 25.000 & 0.3746 & 2.4 & 4.01 \\
\hline & VATT & $B$ & $2 \times 300.0$ & 1.043 & 0.0 & 25.000 & 0.3746 & 2.4 & 4.01 \\
\hline & VATT & $V$ & $2 \times 240.0$ & 1.042 & 0.0 & 25.000 & 0.3746 & 2.3 & 4.01 \\
\hline & VATT & $R$ & $2 \times 180.0$ & 1.120 & 0.0 & 25.000 & 0.3746 & 2.2 & 4.01 \\
\hline MCG $+03-30-071 \ldots$. & UIT & $150 \mathrm{~nm}$ & $1 \times 529.5$ & 0 & 84.124 & 16.928 & 1.200 & 3.03 & 4.21 \\
\hline & UIT & $250 \mathrm{~nm}$ & $1 \times 105.5$ & 0 & 84.125 & 18.658 & 1.200 & 2.71 & 4.21 \\
\hline & $H S T$ & F300W & $2 \times 900.0$ & 0 & 300.002 & 19.433 & 0.0996 & 0.04 & $3 / 4.21$ \\
\hline & $H S T$ & F814W & $2 \times 100.0$ & 0 & 300.002 & 21.659 & 0.0996 & 0.08 & $3 / 4.21$ \\
\hline & VATT & $U$ & $2 \times 600.0$ & 1.045 & 0.0 & 25.000 & 0.3746 & 2.4 & 4.21 \\
\hline & VATT & $B$ & $2 \times 300.0$ & 1.043 & 0.0 & 25.000 & 0.3746 & 2.4 & 4.21 \\
\hline & VATT & $V$ & $2 \times 240.0$ & 1.042 & 0.0 & 25.000 & 0.3746 & 2.3 & 4.21 \\
\hline & VATT & $R$ & $2 \times 180.0$ & 1.120 & 0.0 & 25.000 & 0.3746 & 2.2 & 4.21 \\
\hline NGC $3860 \mathrm{~B}$. & UIT & $150 \mathrm{~nm}$ & $1 \times 529.5$ & 0 & 84.124 & 16.928 & 1.200 & 3.03 & 4.24 \\
\hline & UIT & $250 \mathrm{~nm}$ & $1 \times 105.5$ & 0 & 84.125 & 18.658 & 1.200 & 2.71 & 4.24 \\
\hline & $H S T$ & F300W & $2 \times 900.0$ & 0 & 287.781 & 19.433 & 0.0996 & 0.04 & $3 / 4.24$ \\
\hline & $H S T$ & F814W & $2 \times 100.0$ & 0 & 287.781 & 21.659 & 0.0996 & 0.08 & $3 / 4.24$ \\
\hline & VATT & $U$ & $2 \times 600.0$ & 1.036 & 0.0 & 25.000 & 0.3746 & 1.5 & 4.24 \\
\hline & VATT & $B$ & $2 \times 300.0$ & 1.035 & 0.0 & 25.000 & 0.3746 & 1.6 & 4.24 \\
\hline & VATT & $V$ & $2 \times 240.0$ & 1.034 & 0.0 & 25.000 & 0.3746 & 1.7 & 4.24 \\
\hline & VATT & $R$ & $2 \times 180.0$ & 1.166 & 0.0 & 25.000 & 0.3746 & 2.4 & 4.24 \\
\hline NGC 3921 (Arp 224, Mrk 430).................... & $H S T$ & F300W & $2 \times 950.0$ & 0 & 241.061 & 19.433 & 0.0996 & 0.04 & $3 / 4.07$ \\
\hline & $H S T$ & F814W & $2 \times 130.0$ & 0 & 241.061 & 21.659 & 0.0996 & 0.08 & $3 / 4.07$ \\
\hline
\end{tabular}


TABLE 2-Continued

\begin{tabular}{|c|c|c|c|c|c|c|c|c|c|}
\hline $\begin{array}{l}\text { Object } \\
\text { (1) }\end{array}$ & $\begin{array}{c}\text { Telescope } \\
\text { (2) }\end{array}$ & $\begin{array}{l}\text { Filter } \\
(3)\end{array}$ & $\begin{array}{l}\text { Exposure Time } \\
\text { (s) } \\
\text { (4) }\end{array}$ & $\begin{array}{c}\text { Air Mass } \\
(5)\end{array}$ & $\begin{array}{l}\text { Orientation } \\
\text { (deg) } \\
\text { (6) }\end{array}$ & $\begin{array}{l}\text { Zero Point } \\
\text { (mag) } \\
\text { (7) }\end{array}$ & $\begin{array}{c}\text { Plate Scale } \\
\left(\operatorname{arcsec} \text { pixel }^{-1}\right) \\
(8)\end{array}$ & $\begin{array}{c}\text { FWHM } \\
(\operatorname{arcsec}) \\
(9)\end{array}$ & $\begin{array}{l}\text { Figure } \\
(10)\end{array}$ \\
\hline & $\mathrm{UH} 2.2 \mathrm{~m}$ & $B$ & $2 \times 600.0$ & 1.237 & 359.869 & $\ldots$ & 0.4400 & $?$ & 4.07 \\
\hline & $\mathrm{UH} 2.2 \mathrm{~m}$ & $V$ & $1 \times 900.0$ & 1.250 & 359.869 & $\ldots$ & 0.4400 & $?$ & 4.07 \\
\hline & $\mathrm{UH} 2.2 \mathrm{~m}$ & $R$ & $1 \times 600.0$ & 1.231 & 359.869 & $\ldots$ & 0.4400 & $?$ & 4.07 \\
\hline & $H S T$ & F110W & $1 \times 160.0$ & 0 & 18.507 & 21.100 & 0.0758 & 0.11 & 4.07 \\
\hline & $H S T$ & F160W & $1 \times 256.0$ & 0 & 18.839 & 21.100 & 0.2000 & 0.20 & 4.07 \\
\hline & $\mathrm{UH} 2.2 \mathrm{~m}$ & $K^{\prime}$ & $3 \times 120.0$ & 1.231 & 0.006 & $\ldots$ & 0.1850 & $?$ & 4.07 \\
\hline \multirow{8}{*}{ 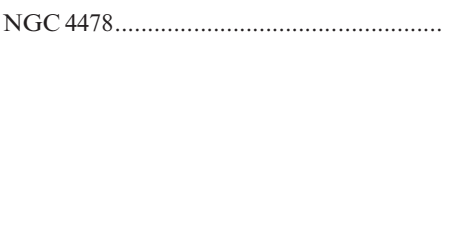 } & UIT & $150 \mathrm{~nm}$ & $1 \times 352.5$ & 0 & 303.985 & 16.928 & 1.200 & 3.03 & 4.03 \\
\hline & UIT & $250 \mathrm{~nm}$ & $1 \times 70.5$ & 0 & 303.985 & 18.658 & 1.200 & 2.71 & 4.03 \\
\hline & $H S T$ & F300W & $2 \times 900.0$ & 0 & 123.922 & 19.433 & 0.0996 & 0.04 & $3 / 4.03$ \\
\hline & $H S T$ & F814W & $2 \times 100.0$ & 0 & 123.922 & 21.659 & 0.0996 & 0.08 & $3 / 4.03$ \\
\hline & VATT & U & $1 \times 300.0$ & 1.072 & 0.0 & $\ldots$ & 0.3746 & 2.31 & 4.03 \\
\hline & VATT & $B$ & $1 \times 300.0$ & 1.070 & 0.0 & $\ldots$ & 0.3746 & 2.10 & 4.03 \\
\hline & VATT & $V$ & $1 \times 200.0$ & 1.066 & 0.0 & $\ldots$ & 0.3746 & 1.62 & 4.03 \\
\hline & VATT & $R$ & $1 \times 120.0$ & 1.068 & 0.0 & $\ldots$ & 0.3746 & 1.70 & 4.03 \\
\hline \multirow{7}{*}{ IC 3949 . } & UIT & $150 \mathrm{~nm}$ & $1 \times 1900.5$ & 0 & 211.560 & 16.806 & 1.1367 & 3.03 & 4.18 \\
\hline & $H S T$ & F300W & $2 \times 900.0$ & 0 & 130.973 & 19.433 & 0.0996 & 0.04 & $3 / 4.18$ \\
\hline & $H S T$ & F814W & $2 \times 100.0$ & 0 & 130.973 & 21.659 & 0.0996 & 0.08 & $3 / 4.18$ \\
\hline & VATT & $U$ & $1 \times 300.0$ & 1.392 & 0.0 & $\ldots$ & 0.3746 & 2.25 & 4.18 \\
\hline & VATT & $B$ & $1 \times 180.0$ & 1.487 & 0.0 & $\ldots$ & 0.3746 & 1.77 & 4.18 \\
\hline & VATT & $V$ & $1 \times 120.0$ & 1.539 & 0.0 & $\ldots$ & 0.3746 & 1.77 & 4.18 \\
\hline & VATT & $R$ & $1 \times 60.0$ & 1.585 & 0.0 & $\ldots$ & 0.3746 & 1.21 & 4.18 \\
\hline \multirow[t]{5}{*}{ UGC 08335 (Arp 238) …............................... } & $H S T$ & F300W & $2 \times 900.0$ & 0 & 234.614 & 19.433 & 0.0996 & 0.04 & $3 / 4.36$ \\
\hline & $H S T$ & F814W & $2 \times 130.0$ & 0 & 234.614 & 21.659 & 0.0996 & 0.08 & $3 / 4.36$ \\
\hline & VATT & $U$ & $2 \times 600.3$ & 1.399 & 0.0 & $\ldots$ & 0.3746 & 1.99 & 4.36 \\
\hline & POSS II & IIIaJ & $1 \times 4500.0$ & $\ldots$ & 0.0 & $\ldots$ & 1.00 & 3.15 & 4.36 \\
\hline & VATT & $R$ & $2 \times 180.1$ & 1.388 & 0.0 & $\ldots$ & 0.3746 & 1.87 & 4.36 \\
\hline \multirow{7}{*}{ Mrk $66 .}$. & UIT & $150 \mathrm{~nm}$ & $1 \times 775.5$ & 0 & 27.310 & 16.806 & 1.1367 & 3.03 & 4.32 \\
\hline & $H S T$ & F300W & $2 \times 900.0$ & 0 & 143.091 & 19.433 & 0.0996 & 0.04 & $3 / 4.32$ \\
\hline & $H S T$ & F814W & $2 \times 130.0$ & 0 & 143.091 & 21.659 & 0.0996 & 0.08 & $3 / 4.32$ \\
\hline & VATT & $U$ & $2 \times 600.1$ & 1.143 & 0.0 & $\ldots$ & 0.3746 & 1.66 & 4.32 \\
\hline & VATT & $B$ & $2 \times 300.0$ & 1.133 & 0.0 & $\ldots$ & 0.3746 & 1.75 & 4.32 \\
\hline & VATT & $V$ & $2 \times 240.8$ & 1.128 & 0.0 & $\ldots$ & 0.3746 & 1.66 & 4.32 \\
\hline & VATT & $R$ & $2 \times 180.0$ & 1.426 & 0.0 & $\ldots$ & 0.3746 & 2.27 & 4.32 \\
\hline \multirow[t]{11}{*}{ 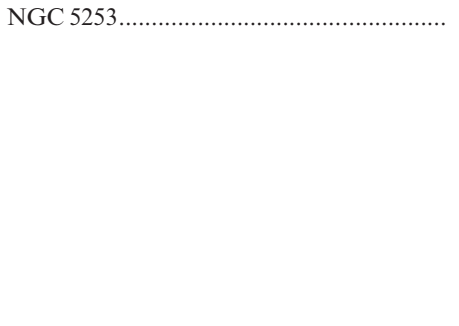 } & UIT & $150 \mathrm{~nm}$ & $2 \times 726.1$ & 0 & 163.350 & 16.806 & 1.1367 & 3.03 & 4.27 \\
\hline & $H S T$ & F255W & $9 \times 766.7$ & 0 & 264.709 & 17.037 & 0.0996 & 0.04 & 4.27 \\
\hline & $H S T$ & F300W & $2 \times 900.0$ & 0 & 291.500 & 19.433 & 0.0996 & 0.04 & $3 / 4.27$ \\
\hline & $H S T$ & F814W & $2 \times 130.0$ & 0 & 291.500 & 21.659 & 0.0996 & 0.08 & $3 / 4.27$ \\
\hline & VATT & $U$ & $5 \times 300.0$ & 2.307 & 0.0 & 25.000 & 0.3746 & 3.3 & 4.27 \\
\hline & VATT & $B$ & $2 \times 300.0$ & 2.304 & 0.0 & 25.000 & 0.3746 & 2.6 & 4.27 \\
\hline & VATT & $V$ & $2 \times 240.0$ & 2.308 & 0.0 & 25.000 & 0.3746 & 2.5 & 4.27 \\
\hline & VATT & $R$ & $2 \times 180.0$ & 2.314 & 0.0 & 25.000 & 0.3746 & 2.3 & 4.27 \\
\hline & $\mathrm{LCO} 2.5 \mathrm{~m}$ & $J$ & $5 \times 50.0$ & 0.500 & 0.0 & 22.20 & 0.3500 & 1.94 & 4.27 \\
\hline & $\mathrm{LCO} 2.5 \mathrm{~m}$ & $H$ & $5 \times 30.0$ & 0.500 & 0.0 & 21.96 & 0.3500 & 1.91 & 4.27 \\
\hline & $\mathrm{LCO} 2.5 \mathrm{~m}$ & $K$ & $9 \times 20.0$ & 0.500 & 0.0 & 21.62 & 0.3500 & 0.98 & 4.27 \\
\hline \multirow{6}{*}{ NGC 5278/79 (Arp 239, Mrk 271) .............. } & $H S T$ & F255W & $3 \times 466.7$ & 0 & 126.600 & 17.037 & 0.0996 & 0.04 & $3 / 4.38$ \\
\hline & $H S T$ & F300W & $3 \times 500.0$ & 0 & 126.600 & 19.433 & 0.0996 & 0.04 & $3 / 4.38$ \\
\hline & $H S T$ & F814W & $2 \times 130.0$ & 0 & 126.600 & 21.659 & 0.0996 & 0.08 & $3 / 4.38$ \\
\hline & VATT & U & $4 \times 300.7$ & 1.496 & 0.0 & $\ldots$ & 0.3746 & 2.49 & 4.38 \\
\hline & POSS II & IIIaJ & $1 \times 3900.0$ & $\ldots$ & 0.0 & $\ldots$ & 1.00 & 3.4 & 4.38 \\
\hline & $\mathrm{UH} 2.2 \mathrm{~m}$ & $R$ & $1 \times 600.0$ & 1.352 & 359.684 & $\ldots$ & 0.4400 & $\ldots$ & 4.38 \\
\hline UGC 08696 (Mrk 273).. & $H S T$ & F255W & $3 \times 466.7$ & 0 & 254.413 & 17.037 & 0.0996 & 0.04 & $3 / 4.09$ \\
\hline & $H S T$ & F300W & $3 \times 500.0$ & 0 & 254.413 & 19.433 & 0.0996 & 0.04 & $3 / 4.09$ \\
\hline & $H S T$ & F814W & $2 \times 130.0$ & 0 & 254.413 & 21.659 & 0.0996 & 0.08 & $3 / 4.09$ \\
\hline & VATT & $U$ & $2 \times 303.8$ & 1.645 & 0.0 & $\ldots$ & 0.3746 & 2.47 & 4.09 \\
\hline & $\mathrm{UH} 2.2 \mathrm{~m}$ & $R$ & $1 \times 600.0$ & 1.377 & 359.684 & $\ldots$ & 0.4400 & $\ldots$ & 4.09 \\
\hline & $H S T$ & F110W & $4 \times 63.9$ & 0 & 88.267 & 24.818 & 0.0758 & 0.11 & 4.09 \\
\hline & $H S T$ & F160W & $4 \times 63.9$ & 0 & 88.267 & 25.447 & 0.0758 & 0.16 & 4.09 \\
\hline & $H S T$ & $\mathrm{~F} 222 \mathrm{M}$ & $4 \times 79.9$ & 0 & 88.267 & 25.131 & 0.0758 & 0.22 & 4.09 \\
\hline UGC 08823 (Mrk 279) ... & UIT & $150 \mathrm{~nm}$ & $2 \times 777.5$ & 0 & 39.999 & 16.806 & 1.1367 & 3.03 & 4.06 \\
\hline & $H S T$ & F300W & $2 \times 950.0$ & 0 & 318.595 & 19.433 & 0.0996 & 0.04 & $3 / 4.06$ \\
\hline & $H S T$ & F814W & $2 \times 160.0$ & 0 & 318.595 & 21.659 & 0.0996 & 0.08 & $3 / 4.06$ \\
\hline & VATT & $U$ & $4 \times 300.0$ & 1.333 & 0.0 & 25.000 & 0.3746 & 3.2 & 4.06 \\
\hline & VATT & $B$ & $2 \times 300.0$ & 1.316 & 0.0 & 25.000 & 0.3746 & 3.1 & 4.06 \\
\hline & VATT & $V$ & $2 \times 240.0$ & 1.317 & 0.0 & 25.000 & 0.3746 & 2.7 & 4.06 \\
\hline & VATT & $R$ & $2 \times 180.0$ & 1.317 & 0.0 & 25.000 & 0.3746 & 2.6 & 4.06 \\
\hline IC 4393. & $H S T$ & F300W & $2 \times 900.0$ & 0 & 150.001 & 19.433 & 0.0996 & 0.04 & $3 / 4.20$ \\
\hline & $H S T$ & F814W & $2 \times 130.0$ & 0 & 150.001 & 21.659 & 0.0996 & 0.08 & $3 / 4.20$ \\
\hline & VATT & $U$ & $2 \times 600.0$ & 2.286 & 0.0 & 25.000 & 0.3746 & 2.4 & 4.20 \\
\hline & VATT & $B$ & $2 \times 300.0$ & 2.291 & 0.0 & 25.000 & 0.3746 & 2.3 & 4.20 \\
\hline & VATT & $V$ & $2 \times 240.0$ & 2.297 & 0.0 & 25.000 & 0.3746 & 2.2 & 4.20 \\
\hline & VATT & $R$ & $2 \times 180.0$ & 2.304 & 0.0 & 25.000 & 0.3746 & 2.1 & 4.20 \\
\hline & ESO $1.5 \mathrm{~m}$ & $I$ & $2 \times 1200.0$ & $\ldots$ & 1.46 & 20.928 & 0.36 & 1.2 & 4.20 \\
\hline & $\mathrm{ESO} 2.2 \mathrm{~m}$ & $K$ & $24 \times 10.0$ & $\ldots$ & 0.11 & 20.772 & 0.491 & 1.4 & 4.20 \\
\hline UGC $09855 \ldots \ldots$ & $H S T$ & F255W & $3 \times 466.7$ & 0 & 291.667 & 17.037 & 0.0996 & 0.04 & $3 / 4.30$ \\
\hline & $H S T$ & F300W & $3 \times 500.0$ & 0 & 291.667 & 19.433 & 0.0996 & 0.04 & $3 / 4.30$ \\
\hline
\end{tabular}


TABLE 2-Continued

\begin{tabular}{|c|c|c|c|c|c|c|c|c|c|}
\hline $\begin{array}{l}\text { Object } \\
\text { (1) }\end{array}$ & $\begin{array}{c}\text { Telescope } \\
\text { (2) }\end{array}$ & $\begin{array}{c}\text { Filter } \\
(3)\end{array}$ & $\begin{array}{l}\text { Exposure Time } \\
\text { (s) } \\
\text { (4) }\end{array}$ & $\begin{array}{c}\text { Air Mass } \\
(5)\end{array}$ & $\begin{array}{c}\text { Orientation } \\
\text { (deg) } \\
\text { (6) }\end{array}$ & $\begin{array}{l}\text { Zero Point } \\
\text { (mag) } \\
(7)\end{array}$ & $\begin{array}{c}\text { Plate Scale } \\
\left(\operatorname{arcsec} \text { pixel }^{-1}\right) \\
(8)\end{array}$ & $\begin{array}{c}\text { FWHM } \\
(\operatorname{arcsec}) \\
(9)\end{array}$ & $\begin{array}{c}\text { Figure } \\
(10)\end{array}$ \\
\hline & $H S T$ & F814W & $2 \times 130.0$ & 0 & 291.667 & 21.659 & 0.0996 & 0.08 & $3 / 4.30$ \\
\hline & VATT & $U$ & $2 \times 600.0$ & 1.234 & 0.0 & 25.000 & 0.3746 & 2.7 & 4.30 \\
\hline & VATT & $B$ & $2 \times 240.0$ & 1.255 & 0.0 & 25.000 & 0.3746 & 2.9 & 4.30 \\
\hline & VATT & $V$ & $2 \times 300.0$ & 1.234 & 0.0 & 25.000 & 0.3746 & 2.5 & 4.30 \\
\hline & VATT & $R$ & $2 \times 180.0$ & 1.264 & 0.0 & 25.000 & 0.3746 & 2.7 & 4.30 \\
\hline \multirow[t]{6}{*}{ 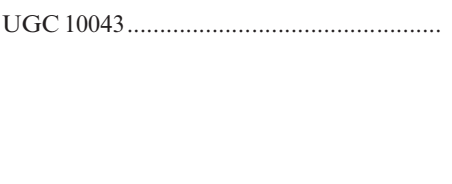 } & $H S T$ & F300W & $2 \times 900.0$ & 0 & 250.001 & 19.433 & 0.0996 & 0.04 & $3 / 4.16$ \\
\hline & $H S T$ & F814W & $2 \times 100.0$ & 0 & 250.001 & 21.659 & 0.0996 & 0.08 & $3 / 4.16$ \\
\hline & VATT & $U$ & $2 \times 1800.0$ & 1.048 & 0.0 & 25.000 & 0.3746 & 1.5 & 4.16 \\
\hline & VATT & $B$ & $2 \times 1200.0$ & 1.158 & 0.0 & 25.000 & 0.3746 & 1.3 & 4.16 \\
\hline & VATT & $V$ & $2 \times 900.0$ & 1.167 & 0.0 & 25.000 & 0.3746 & 1.1 & 4.16 \\
\hline & VATT & $R$ & $2 \times 600.0$ & 1.331 & 0.0 & 25.000 & 0.3746 & 1.2 & 4.16 \\
\hline \multirow{9}{*}{ NGC 6753.} & $H S T$ & F300W & $2 \times 950.0$ & 0 & 154.222 & 19.433 & 0.0996 & 0.04 & $3 / 4.11$ \\
\hline & $H S T$ & $\mathrm{~F} 814 \mathrm{~W}$ & $2 \times 160.0$ & 0 & 154.222 & 21.659 & 0.0996 & 0.08 & $3 / 4.11$ \\
\hline & CTIO $0.9 \mathrm{~m}$ & $U$ & $1 \times 1200.0$ & 1.125 & 0.0 & $\ldots$ & 0.396 & 1.45 & 4.11 \\
\hline & CTIO $0.9 \mathrm{~m}$ & $B$ & $4 \times 300.0$ & 1.382 & 0.0 & $\ldots$ & 0.396 & 1.68 & 4.11 \\
\hline & CTIO $0.9 \mathrm{~m}$ & $V$ & $3 \times 300.0$ & 1.453 & 0.0 & $\ldots$ & 0.396 & 1.76 & 4.11 \\
\hline & CTIO $0.9 \mathrm{~m}$ & $R$ & $3 \times 200.0$ & 1.514 & 0.0 & $\ldots$ & 0.396 & 1.90 & 4.11 \\
\hline & CTIO $1.5 \mathrm{~m}$ & $J$ & $\ldots$ & $\ldots$ & 0.0 & $\ldots$ & 0.645 & 1.11 & 4.11 \\
\hline & CTIO $1.5 \mathrm{~m}$ & $H$ & $\ldots$ & $\ldots$ & 0.0 & $\ldots$ & 0.645 & 1.25 & 4.11 \\
\hline & CTIO $1.5 \mathrm{~m}$ & $K$ & $\ldots$ & . & 0.0 & $\ldots$ & 0.645 & 1.10 & 4.11 \\
\hline \multirow{7}{*}{ 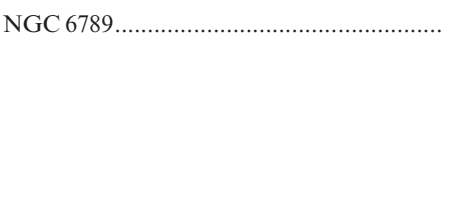 } & $H S T$ & F255W & $3 \times 466.7$ & 0 & 349.145 & 17.037 & 0.0996 & 0.04 & $3 / 4.31$ \\
\hline & $H S T$ & F300W & $3 \times 500.0$ & 0 & 349.145 & 19.433 & 0.0996 & 0.04 & $3 / 4.31$ \\
\hline & $H S T$ & F814W & $2 \times 130.0$ & 0 & 349.145 & 21.659 & 0.0996 & 0.08 & $3 / 4.31$ \\
\hline & VATT & $U$ & $2 \times 600.0$ & 1.674 & 0.0 & 25.000 & 0.3746 & 2.1 & 4.31 \\
\hline & VATT & $B$ & $2 \times 300.0$ & 1.676 & 0.0 & 25.000 & 0.3746 & 2.0 & 4.31 \\
\hline & VATT & $V$ & $2 \times 240.0$ & 1.586 & 0.0 & 25.000 & 0.3746 & 1.8 & 4.31 \\
\hline & VATT & $R$ & $2 \times 180.0$ & 1.657 & 0.0 & 25.000 & 0.3746 & 1.5 & 4.31 \\
\hline \multirow[t]{10}{*}{ 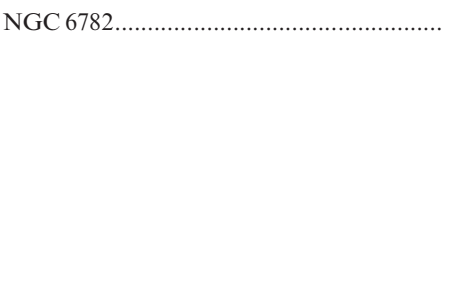 } & $H S T$ & F255W & $3 \times 466.7$ & 0 & 151.206 & 17.037 & 0.0996 & 0.04 & $3 / 4.15$ \\
\hline & $H S T$ & F300W & $3 \times 500.0$ & 0 & 151.206 & 19.433 & 0.0996 & 0.04 & $3 / 4.15$ \\
\hline & $H S T$ & F814W & $2 \times 130.0$ & 0 & 151.206 & 21.659 & 0.0996 & 0.08 & $3 / 4.15$ \\
\hline & CTIO $0.9 \mathrm{~m}$ & U & $2 \times 2400.0$ & 1.154 & 0.0 & $\ldots$ & 0.396 & 2.09 & 4.15 \\
\hline & CTIO $0.9 \mathrm{~m}$ & $B$ & $4 \times 450.0$ & 1.321 & 0.0 & $\ldots$ & 0.396 & 1.59 & 4.15 \\
\hline & CTIO $0.9 \mathrm{~m}$ & $V$ & $3 \times 300.0$ & 1.399 & 0.0 & $\ldots$ & 0.396 & 1.37 & 4.15 \\
\hline & CTIO $0.9 \mathrm{~m}$ & $R$ & $3 \times 200.0$ & 1.448 & 0.0 & $\ldots$ & 0.396 & 1.38 & 4.15 \\
\hline & CTIO $1.5 \mathrm{~m}$ & $J$ & $2 \times 20.0$ & $\ldots$ & 0.0 & $\ldots$ & 0.645 & 1.33 & 4.15 \\
\hline & CTIO $1.5 \mathrm{~m}$ & $H$ & $2 \times 10.0$ & $\ldots$ & 0.0 & $\ldots$ & 0.645 & 1.31 & 4.15 \\
\hline & KPNO $2.2 \mathrm{~m}$ & $K$ & $\ldots$ & $\ldots$ & 0.0 & $\ldots$ & 1.09 & 1.72 & 4.15 \\
\hline \multirow[t]{8}{*}{ NGC 7685... } & $H S T$ & F300W & $2 \times 900.0$ & 0 & 64.488 & 19.433 & 0.0996 & 0.04 & $3 / 4.14$ \\
\hline & $H S T$ & F814W & $2 \times 100.0$ & 0 & 64.488 & 21.659 & 0.0996 & 0.08 & $3 / 4.14$ \\
\hline & VATT & $U$ & $2 \times 600.0$ & 1.261 & 0.0 & $\ldots$ & 0.3746 & 1.59 & 4.14 \\
\hline & VATT & $B$ & $1 \times 300.0$ & 1.286 & 0.0 & $\ldots$ & 0.3746 & 1.39 & 4.14 \\
\hline & JKT & $V$ & $1 \times 346.8$ & 1.102 & 0.0 & 22.322 & 0.30 & 1.39 & 4.14 \\
\hline & JKT & $R$ & $1 \times 289.0$ & 1.106 & 0.0 & 22.558 & 0.30 & 1.63 & 4.14 \\
\hline & JKT & $I$ & $1 \times 289.0$ & 1.114 & 0.0 & 21.833 & 0.30 & 1.21 & 4.14 \\
\hline & UKIRT & $K$ & $8 \times 75.0$ & 1.057 & 0.0 & 20.018 & 0.30 & 1.55 & 4.14 \\
\hline \multirow[t]{8}{*}{ 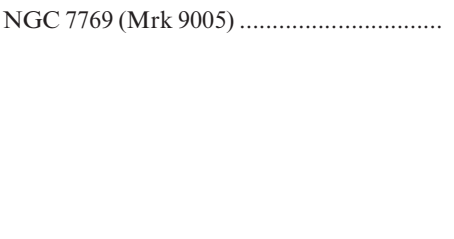 } & $H S T$ & F300W & $2 \times 900.0$ & 0 & 64.927 & 19.433 & 0.0996 & 0.04 & $3 / 4.12$ \\
\hline & $H S T$ & F814W & $2 \times 100.0$ & 0 & 64.927 & 21.659 & 0.0996 & 0.08 & $3 / 4.12$ \\
\hline & VATT & $U$ & $2 \times 600.0$ & 1.025 & 0.0 & $\ldots$ & 0.3746 & 1.42 & 4.12 \\
\hline & VATT & $B$ & $2 \times 300.0$ & 1.026 & 0.0 & $\ldots$ & 0.3746 & 1.34 & 4.12 \\
\hline & VATT & $V$ & $1 \times 300.0$ & 1.086 & 0.0 & $\ldots$ & 0.3746 & 0.97 & 4.12 \\
\hline & VATT & $R$ & $1 \times 300.0$ & 1.059 & 0.0 & $\ldots$ & 0.3746 & 1.10 & 4.12 \\
\hline & JKT & $I$ & $1 \times 404.6$ & 1.035 & 0.0 & 21.709 & 0.30 & 1.36 & 4.12 \\
\hline & UKIRT & $H$ & $5 \times 60.0$ & 1.000 & 0.0 & 20.500 & 0.30 & 1.41 & 4.12 \\
\hline
\end{tabular}

Note.-Col. (1): Galaxy name. Col. (2): Telescope that produced the listed images. Col. (3): Filter: HST/WFPC2 (see Biretta et al. 2000), UIT (see Stecher et al. 1997; $150 \mathrm{~nm}$ denotes both B1 and B2, $250 \mathrm{~nm}$ both A1 and A5 filters), or ground-based Johnson UBV and Kron-Cousins RIJHK. Col. (4): Total exposure time, given as the number of independent integrations times the single image exposure time (as listed in the FITS headers and corresponding to the zero points listed in col. [7]). For unequal length exposures, the listed single-exposure time is the mean of the actual exposure times. Col. (5): Average air mass of the image stack (0 for space-based observations). Col. (6): Image orientation. For HST, this is angle PA_V3 (measured from north through east) of the V3 axis (i.e., the $X=Y$ diagonal of the WFC3 CCD $+180^{\circ}$ ). For UIT and ground-based images, the P.A. of the $Y$-axis of the CCD image is given (i.e., P.A. $=0$ means that north is up and east is to the left). Col. (7): Photometric zero point (ZP) derived for the ground-based images using Vega $\operatorname{mag}=\mathrm{ZP}-2.5 \log _{10}(\mathrm{DN} / t)$, with DN the total object flux and $t$ in seconds (see col. [4]). For HST/WFPC2, these are the Holtzman et al. 1995 Vega zero points, and for UIT, $\mathrm{ZP}_{\mathrm{ST}}=-2.5 \log _{10} C-21.1$, with $C$ the calibration constants $C_{\mathrm{NUV}}$ and $C_{\mathrm{FUV}}$ listed in Stecher et al. 1997. We only list available and confirmed zero points but will update the FITS headers in the public database as ground-based calibrations become available (see V. A. Taylor et al. 2003, in preparation). Col. (8): Image pixel scale. Col. (9): Estimated FWHM of the relevant image PSF in arcseconds. For HST images, these are not to be confused with the 0.0996 WFC pixel scale and are derived as FWHM $=206265^{\prime \prime} \times 1.22 \lambda_{\text {cent }} / D$, with the central wavelength of the filter (see text) in meters and primary aperture $D=2.4 \mathrm{~m}$. Note that especially the WFPC2 UV images are badly undersampled. Microroughness of the $H S T$ mirror limits the mid-UV PSF to $\sim 0$ ".04 FWHM. For ground-based images, the FWHM values were measured using unsaturated stellar objects in each image as identified using SExtractor. Col. (10): Number of the figure(s) displaying each image. 
TABLE 3

Other Imaging Data in the $H S T$ Archive on Our Cycle 9 Galaxy Sample

\begin{tabular}{|c|c|c|c|c|c|c|c|c|}
\hline \multirow[t]{2}{*}{ ESO 418-G008 .......... } & F606W & 5957 & 1508 & $2 \times 80$ & 227.216 & SNAP 5446 & G. Illingworth & Center in PCl \\
\hline & F814W & 7940 & 1531 & $2 \times 600$ & 16.701 & SNAP 8599 & T. Böker & $\mathrm{PC} 1$ \\
\hline \multirow[t]{2}{*}{ NGC $1510 \ldots \ldots \ldots \ldots . .}$. & F170W & 1730 & 500 & $2 \times 400$ & 303.570 & GO 6716 & T. Stecher & \\
\hline & F814W & 7940 & 1531 & $1 \times 200$ & 303.570 & & & \\
\hline UGC $03426 \ldots \ldots \ldots \ldots \ldots$ & F606W & 5957 & 1508 & $1 \times 500$ & 73.315 & SNAP 5479 & M. Malkan & $\mathrm{PC} 1$ \\
\hline \multirow[t]{2}{*}{ IC $2184 \ldots \ldots \ldots \ldots \ldots \ldots$} & F555W & 5407 & 1236 & $1 \times 700+1 \times 400$ & 94.381 & GTO 6870 & J. Trauger & \\
\hline & F814W & 7940 & 1531 & $2 \times 600$ & 94.381 & & & \\
\hline UGC $05101 \ldots \ldots \ldots \ldots \ldots$ & F814W & 7940 & 1531 & $2 \times 400$ & 321.786 & SNAP 6346 & K. Borne & \\
\hline \multirow{2}{*}{ NGC $3310 \ldots \ldots \ldots \ldots \ldots . . . . .}$. & F606W & 5957 & 1508 & $1 \times 500$ & 280.821 & SNAP 5479 & M. Malkan & $\mathrm{PC} 1$ \\
\hline & F336W & 3344 & 381 & $2 \times 500$ & 350.875 & GO 6639 & G. Meurer & $\mathrm{PC} 1$ \\
\hline & F547M & 5479 & 486 & $2 \times 70$ & 28.496 & GO 6419 & A. Wilson & $\mathrm{PCl}$ \\
\hline & F791W & 7829 & 1218 & $2 \times 70$ & 28.465 & & & \\
\hline & $\mathrm{F} 450 \mathrm{~W}$ & 4520 & 958 & $1 \times 900+1 \times 1400$ & 12.393 & GO 6633 & C. Carollo & $\mathrm{PCl}$ \\
\hline & F555W & 5407 & 1236 & $1 \times 300+1 \times 700$ & 12.393 & & & \\
\hline & F814W & 7940 & 1531 & $1 \times 230+1 \times 500$ & 12.393 & & & \\
\hline \multirow[t]{7}{*}{ NGC 3690/IC $694 \ldots$} & F606W & 5957 & 1508 & $1 \times 500$ & 177.915 & SNAP 5479 & M. Malkan & $\mathrm{PC} 1$ \\
\hline & F336W & 3344 & 381 & $4 \times 400$ & 142.669 & GO 6639 & G. Meurer & $\mathrm{PC} 1$ \\
\hline & F439W & 4300 & 475 & $2 \times 200+2 \times 260$ & 142.669 & & & \\
\hline & F814W & 7940 & 1531 & $2 \times 40+160+260$ & 142.669 & & & \\
\hline & F555W & 5407 & 1236 & $2 \times 350$ & 306.258 & SNAP 8602 & A. Filippenko & PC1 \\
\hline & F814W & 7940 & 1531 & $2 \times 350$ & 356.266 & & & \\
\hline & F814W & 7940 & 1531 & $2 \times 350$ & 293.276 & & & \\
\hline UGC $06527 \ldots \ldots \ldots \ldots \ldots$ & F606W & 5957 & 1508 & $1 \times 500$ & 157.556 & SNAP 5479 & M. Malkan & $\mathrm{PC} 1$ \\
\hline NGC $3921 \ldots \ldots \ldots \ldots \ldots$ & F336W & 3344 & 381 & $2 \times 600$ & 172.058 & GO 5416 & B. Whitmore & $\mathrm{PC} 1$ \\
\hline & F814W & 7940 & 1531 & $2 \times 1700$ & 291.727 & & & \\
\hline & F702W & 6895 & 1389 & $1 \times 300+1 \times 700$ & 325.004 & SNAP 6357 & W. Jaffe & PC1 \\
\hline & F555W & 5407 & 1236 & $4 \times 400$ & 291.943 & GO 6587 & D. Richstone & $\mathrm{PCl}$ \\
\hline & F814W & 7940 & 1531 & $2 \times 800$ & 291.943 & & & \\
\hline \multirow[t]{12}{*}{ NGC $5253 \ldots \ldots \ldots \ldots \ldots . .}$. & F606W & 5957 & 1508 & $1 \times 500$ & 131.324 & SNAP 5479 & M. Malkan & $\mathrm{PCl}$ \\
\hline & F255W & 2553 & 393 & $3 \times 800+3 \times 800+3 \times 700$ & 264.709 & GO 6124 & R. Fesen & \\
\hline & F547M & 5479 & 486 & $2 \times 200+2 \times 600$ & 264.460 & GO 6524 & D. Calzetti & \\
\hline & F656N & 6563 & 21 & $2 \times 500+1100+1500$ & 264.460 & & & \\
\hline & F814W & 7940 & 1531 & $2 \times 180+2 \times 400$ & 264.460 & & & \\
\hline & F487N & 4866 & 26 & $3 \times 1300+1 \times 1200$ & 264.460 & & & \\
\hline & F170W & 1730 & 500 & $2 \times 400$ & 280.005 & GO 6716 & T. Stecher & $\mathrm{PC} 1$ \\
\hline & F336W & 3344 & 381 & $2 \times 260$ & 280.005 & & & \\
\hline & F555W & 5407 & 1236 & $1 \times 200$ & 280.005 & & & \\
\hline & F814W & 7940 & 1531 & $1 \times 200$ & 280.005 & & & \\
\hline & F502N & 5013 & 27 & $200+260+600+800$ & 259.612 & GO 9144 & D. Calzetti & \\
\hline & F673N & 6732 & 47 & $2 \times 600+1 \times 1200$ & 259.612 & & & \\
\hline \multirow[t]{2}{*}{ UGC $08696 \ldots \ldots \ldots \ldots . .}$. & F814W & 7940 & 1531 & $2 \times 400$ & 343.721 & SNAP 6346 & K. Borne & PC1 \\
\hline & F814W & 7940 & 1531 & $2 \times 400$ & 20.558 & & & WF3 \\
\hline UGC $08823 \ldots \ldots \ldots \ldots . .$. & F606W & 5957 & 1508 & $1 \times 500$ & 182.130 & SNAP 5479 & M. Malkan & $\mathrm{PC} 1$; core saturated \\
\hline 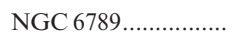 & F336W & 3344 & 381 & $2 \times 1400$ & 303.896 & GO 8122 & R. Schulte-Ladbeck & $\mathrm{PCl}$ \\
\hline & F555W & 5407 & 1236 & $2 \times 1300+4 \times 1400$ & 303.896 & & & \\
\hline & F656N & 6563 & 21 & $2 \times 1400$ & 303.896 & & & \\
\hline & F814W & 7940 & 1531 & $2 \times 1300+4 \times 1400$ & 303.896 & & & \\
\hline NGC $6782 \ldots \ldots \ldots \ldots \ldots . .$. & F450W & 4520 & 958 & $3 \times 300$ & 151.212 & $\mathrm{GO} / \mathrm{DD} 8550$ & K. Noll & \\
\hline & F606W & 5957 & 1508 & $2 \times 160$ & 151.212 & & & \\
\hline & F814W & 7940 & 1531 & $2 \times 160$ & 151.212 & & & \\
\hline
\end{tabular}


TABLE 3-Continued

\begin{tabular}{|c|c|c|c|c|c|c|c|c|}
\hline $\begin{array}{l}\text { Object } \\
\text { (1) }\end{array}$ & $\begin{array}{l}\text { Filter } \\
(2)\end{array}$ & $\begin{array}{l}\lambda_{c} \\
(\AA) \\
(3)\end{array}$ & $\begin{array}{l}\Delta \lambda \\
(\AA) \\
(4)\end{array}$ & $\begin{array}{c}\text { Exposure } \\
\text { Time (s) } \\
\text { (5) }\end{array}$ & $\begin{array}{c}\text { Orientation } \\
\text { (deg) } \\
\text { (6) }\end{array}$ & $\begin{array}{c}\text { Proposal ID } \\
\text { (7) }\end{array}$ & $\begin{array}{l}\text { PI } \\
(8)\end{array}$ & $\begin{array}{l}\text { Note } \\
(9)\end{array}$ \\
\hline \multicolumn{9}{|c|}{ NICMOS Images } \\
\hline \multirow[t]{3}{*}{ UGC $05101 \ldots \ldots \ldots \ldots . .}$. & F110W & 11285 & 1632 & $1 \times 112$ & 107.171 & GTO 7219 & N. Scoville & $\mathrm{NIC} 2$ \\
\hline & F160W & 16060 & 1177 & $1 \times 112$ & 107.171 & & & \\
\hline & F220M & 22182 & 512 & $1 \times 136$ & 107.171 & & & \\
\hline \multirow[t]{6}{*}{ NGC $3310 \ldots \ldots \ldots \ldots . . . . . . .}$. & F110W & 11292 & 1654 & $1 \times 128$ & 125.84 & GO 7268 & R. van der Marel & $\mathrm{NIC1}$ \\
\hline & F110W & 11285 & 1632 & $1 \times 160$ & 125.84 & & & $\mathrm{NIC} 2$ \\
\hline & F160W & 16071 & 1170 & $2 \times 256$ & 125.84 & & & $\mathrm{NIC1}$ \\
\hline & F160W & 16060 & 1177 & $1 \times 128+1 \times 192$ & 125.84 & & & $\mathrm{NIC} 2$ \\
\hline & F160W & 16078 & 1172 & $1 \times 128+1 \times 256$ & 125.84 & & & $\mathrm{NIC} 3$ \\
\hline & F205W & 20714 & 1832 & $1 \times 256$ & 125.84 & & & $\mathrm{NIC} 2$ \\
\hline NGC $3516 \ldots \ldots \ldots \ldots \ldots$ & F160W & 16060 & 1177 & $1 \times 704$ & 251.013 & SNAP 7330 & J. Mulchaey & $\mathrm{NIC} 2$ \\
\hline \multirow[t]{11}{*}{ NGC $3690 /$ IC $694 \ldots$} & F110M & 11026 & 590 & $8 \times 128$ & 133.864 & GTO 7218 & M. Rieke & $\mathrm{NIC} 1$; mosaic of eight pointings \\
\hline & F160W & 16060 & 1177 & $2 \times 104$ & 133.851 & & & NIC2; two pointings \\
\hline & F166N & 16583 & 59 & $1 \times 256$ & 133.901 & & & $\mathrm{NIC} 3$ \\
\hline & $\mathrm{F} 187 \mathrm{~N}$ & 18748 & 67 & $4 \times 640$ & 133.851 & & & $\mathrm{NIC} 1$; mosaic of four pointings \\
\hline & F187N & 18740 & 68 & $2 \times 160$ & 133.851 & & & NIC2; two pointings \\
\hline & F190N & 18986 & 73 & $4 \times 640$ & 133.842 & & & $\mathrm{NIC} 1$; mosaic of four pointings \\
\hline & F190N & 19003 & 63 & $2 \times 160$ & 133.851 & & & NIC2; two pointings \\
\hline & $\mathrm{F} 212 \mathrm{~N}$ & 21213 & 89 & $1 \times 1280+1 \times 1408$ & 133.851 & & & NIC2; two pointings \\
\hline & F237M & 23696 & 473 & $2 \times 288$ & 133.851 & & & NIC2; two pointings \\
\hline & $\mathrm{F} 215 \mathrm{~N}$ & 21487 & 80 & $1 \times 1408+1 \times 1408$ & 133.842 & & & NIC2; two pointings \\
\hline & F222M & 22182 & 512 & $2 \times 176$ & 133.842 & & & NIC2; two pointings \\
\hline UGC $06527 .$. & F160W & 16060 & 1177 & $1 \times 256$ & 39.150 & SNAP 7328 & M. Malkan & $\mathrm{NIC} 2$ \\
\hline \multirow[t]{5}{*}{ NGC $3921 \ldots \ldots \ldots \ldots \ldots . . . . .}$. & F110W & 11285 & 1632 & $1 \times 160$ & 63.944 & GO 7268 & R. van der Marel & $\mathrm{NIC} 2$ \\
\hline & F110W & 11292 & 1654 & $1 \times 128$ & 63.948 & & & $\mathrm{NICl}$ \\
\hline & F160W & 16060 & 1177 & $1 \times 192+1 \times 128$ & 63.944 & & & NIC2; two pointings \\
\hline & F160W & 16078 & 1172 & $2 \times 256+2 \times 128+192$ & 63.944 & & & NIC 3 ; mosaic of four pointings \\
\hline & F205W & 20714 & 1832 & $3 \times 256$ & 63.944 & & & NIC2; three pointings \\
\hline \multirow[t]{7}{*}{ NGC $5253 \ldots \ldots \ldots \ldots \ldots$} & F110W & 11285 & 1632 & $1 \times 24$ & 105.339 & GTO 7219 & N. Scoville & $\mathrm{NIC} 2$ \\
\hline & $\mathrm{F} 187 \mathrm{~N}$ & 18740 & 68 & $1 \times 64$ & 105.339 & & & \\
\hline & F160W & 16060 & 1177 & $1 \times 24$ & 105.339 & & & \\
\hline & F190N & 19003 & 63 & $1 \times 64$ & 105.339 & & & \\
\hline & F222M & 22182 & 512 & $1 \times 80$ & 105.339 & & & \\
\hline & F160W & 16071 & 1170 & $3 \times 384+2 \times 640$ & 296.776 & GO 7849 & W. Freedman & $\mathrm{NIC1}$ \\
\hline & F160W & 16060 & 1177 & $3 \times 384+2 \times 640$ & 296.776 & & & $\mathrm{NIC} 2$ \\
\hline \multirow[t]{3}{*}{ UGC $08696 \ldots \ldots \ldots \ldots . . . .}$. & F110W & 11285 & 1632 & $1 \times 64$ & 133.577 & GTO 7219 & N. Scoville & $\mathrm{NIC} 2$ \\
\hline & F160W & 16060 & 1177 & $1 \times 64$ & 133.577 & & & \\
\hline & F222M & 22182 & 512 & $1 \times 80$ & 133.577 & & & \\
\hline \multicolumn{9}{|c|}{ FOC Images } \\
\hline \multirow[t]{9}{*}{ UGC $03426 \ldots \ldots \ldots \ldots . .}$. & F210M & 2156 & 214 & $1 \times 596$ & 274.030 & GTO 5140 & F. Macchetto & \\
\hline & F320W & 3360 & 844 & $1 \times 596$ & 274.030 & & & \\
\hline & F342W & 3410 & 702 & $1 \times 597$ & 277.678 & & & \\
\hline & F372M & 3710 & 406 & $1 \times 896$ & 274.030 & & & \\
\hline & F502M & 4940 & 530 & $1 \times 750$ & 274.030 & & & \\
\hline & F550M & 5460 & 188 & $1 \times 1196$ & 274.030 & & & \\
\hline & F502M & 4940 & 530 & $3 \times 5262$ & 289.829 & GO 5918 & D. Axon & Imaging polarimetry \\
\hline & F275W & 2770 & 594 & $3 \times 5187$ & 20.342 & GO 6702 & L. Kay & Imaging polarimetry \\
\hline & F342W & 3410 & 702 & $3 \times 1697$ & 20.342 & & & \\
\hline 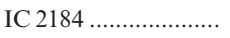 & F220W & 2280 & 480 & $1 \times 996$ & 120.036 & GO 4800 & P. Conti & Pre-refurbishment \\
\hline 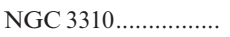 & F220W & 2280 & 480 & $1 \times 197$ & 13.102 & GO 3591 & T. Heckman & Pre-refurbishment \\
\hline \multirow[t]{2}{*}{ NGC $3690 /$ IC $694 \ldots$} & F220W & 2280 & 480 & $1 \times 897$ & 333.653 & GO 3591 & T. Heckman & Pre-refurbishment \\
\hline & F220W & 2280 & 480 & $2 \times 896+2 \times 896$ & 269.868 & GO 3792 & S. Neff & Two pointings \\
\hline NGC $5253 \ldots \ldots \ldots \ldots \ldots$ & F220W & 2280 & 480 & $1 \times 497$ & 122.891 & GO 3591 & T. Heckman & Pre-refurbishment \\
\hline
\end{tabular}

small). Since our WFPC2 mid-UV images are read-noise limited, the resulting one-orbit $1 \sigma \mathrm{SB}$ sensitivity is $25.1 \pm 0.15 \mathrm{mag} \operatorname{arcsec}^{-2}$ in $\mathrm{F} 300 \mathrm{~W}$ and $23.0 \pm 0.15 \mathrm{mag}$ $\operatorname{arcsec}^{-2}$ in $\mathrm{F} 255 \mathrm{~W}$ on a per pixel basis. The relation between the detected SB level and the $\mathrm{S} / \mathrm{N}$ in a pixel is given by $\mathrm{S} / \mathrm{N}=10^{-0.4\left(\mu_{\mathrm{F} 300 \mathrm{~W}}-25.1\right)}$ and $\mathrm{S} / \mathrm{N}=10^{-0.4\left(\mu_{\mathrm{F} 25 \mathrm{~W}}-23.0\right)}$ for F300W and F255W, respectively. These mid-UV SB limits are consistent with the values expected from the Cycle 5-6 images in F410M and F450W (Pascarelle et al. 1996; Odewahn et al. 1996; Windhorst et al. 1998a) and the relatively red color of the zodiacal sky background. Taking into account the $(1+z)^{4} \mathrm{SB}$ dimming and the typical $(U-I)$ color of galaxies at $z \simeq 1-2$, the SB sensitivity reached in the present data matches that achieved for typical faint $I \lesssim 26$ galaxies seen in deep HST images.

The one-orbit $3 \sigma$ point-source sensitivity is $26.4 \pm 0.15$ mag in F300W and $\sim 24.5$ mag in F255W. Hence, many of the galaxies in our sample are resolved into their brightest star-forming regions and (most likely) into their OB associations and young star clusters. This is not true, however, for most of the merging/interacting galaxies that were selected into our sample from the sample of J. E. Hibbard et al. 
(2002, in preparation). Because these systems are relatively rare, they tend to be 2-3 times more distant than the bulk of our sample (see Table 1, col. [14]) and so are not resolved into stars.

\subsubsection{Red Leak}

The F300W filter has a significant red leak, causing a fraction of an object's flux longward of $4000 \AA$ to be detected in this mid-UV filter. Figure 3.10 of the WFPC2 Handbook (Biretta et al. 2001) shows that the red-leak portion of the $\mathrm{QE} \times \mathrm{T}$ curve of the F300W filter resembles the throughput curve of the F814W filter, which transmits mostly photons in the 7000-9000 A range. Table 3.13 of the WFPC2 handbook suggests that the red leak is generally no more than $5 \%$ of the total F300W flux for stellar populations dominated by stars of spectral type K3 V or earlier, although it can be as much as $10 \%-50 \%$ of the total F300W flux for stellar populations dominated by M0-M8 V stars. Hence, even for elliptical galaxies with $\mathrm{K}$ star spectra, the red leak is expected to be relatively small, and for late-type galaxies dominated by young hot stellar populations it should be almost negligible.

For realistic galaxy SEDs, P. B. Eskridge et al. (2002, in preparation) find that the red leak is typically $5 \%-7 \%$ of the total F300W flux and never exceeds $10 \%$ of the F300W flux, not even in the reddest galaxy bulges. We verified this for several red galaxies in our sample by subtracting a fraction of the F814W images from the F300W images, after appropriately rescaling with the relative exposure time and making sure both images were registered the same way. This fraction of the subtracted F814W image amounted to $7 \%$ of the total F300W flux in the brightest region of the galaxy bulge that is presumed to be dominated by G8-K3 stars (following the red leak as modeled by P. B. Eskridge et al. 2002, in preparation).

We found that for the redder stellar populations in those images no noticeable additional structure was introduced in our F300W images at the locations of the brightest F814W flux. To illustrate this, a very red star is seen just above the center of both edge-on galaxies ESO 033-G22 and IC 4394 in the F814W images of Figures 3.19 and 3.20. For IC 4394, Figure 4.20 shows how red this star is, where it is seen just south of the galaxy center. These stars are saturated in the F814W images of both galaxies, and at the corresponding locations in the (non-red-leak-corrected) F300W images of Figures 3.19 and 3.20, only a very faint red-leak flux is seen. These worst-case examples show that the apparent F300W morphology of any of our galaxies would not be significantly affected by the red leak in a few of the very reddest and brightest galaxy areas in the F814W images. Such areas would have to be significantly saturated in our F814W exposures to generate significant red leak in the F300W images, and none of our targeted galaxies were saturated anywhere in the F814W images. Hence, for the current qualitative presentation of the mid-UV images, and given that our sample is biased toward the bluer galaxies, we have thus not corrected the images presented in the mid-UV atlas of $\S 3$ for the small contamination by red leak in the brightest and reddest areas.

For accurate quantitative measurements of galaxy properties we will subtract the red leak in future papers where necessary (e.g., P. B. Eskridge et al. 2002, in preparation). This will be done together with the determination of pixel- to-pixel SEDs for each galaxy, in case there are subtle dependencies of the red leak on the red SED. However, to first order there should be no such dependency, since galaxies of all spectral types show remarkably little change in their relative SEDs between 7000 and $9000 \AA$.

\subsubsection{Data Archiving}

As we expect these data to be of use beyond the scope of our immediate science goals, we will make all images available to the community in digital form when this paper goes to press, even though the photometric zero points for part of the ground-based images are not yet established. We will update the FITS headers as new photometry becomes available (see also V. A. Taylor et al. 2003, in preparation). Hence, the FITS headers in the public database will override any values currently listed in Table 2 . The images will be made public via ADS and also on-line. ${ }^{6}$

Both the HST and ground-based data will be made publicly available at this site. The raw WFPC2 data can be obtained from the HST Archive. Additional information regarding this survey and its planning, the observations, and reduction procedures can be found on-line as well. ${ }^{7}$

\section{PRESENTATION OF THE WFPC2 MID-UV IMAGES}

In this section we describe the HST/WFPC2 images and discuss each individual object in the sample. In view of the large volume of the combined ground- plus space-based data set, we opted to only present the Cycle 9 HST/WFPC2 images in the printed version of the present paper (Fig. 3). The electronic version contains the full " panchromatic atlas of nearby galaxies," which include UIT and ground-based $U B V R(I)$ and $J H K$ images, wherever available (see Fig. 4). Of these, we show in the printed version a few example pages (Fig. 4) to clarify the adopted format.

The galaxies are presented roughly in order of their observed morphological type. We start with the regular early-type galaxies in $\S 3.1$ (and correspondingly numbered 3.01-3.03 and 4.01-4.03). Subsequent paragraphs present early-type galaxies with significant AGNs in the mid-UV (§ 3.2) and merger products that are likely to evolve into early-type remnants ( $\S 3.3)$. Next, we discuss early- through mid-type spiral galaxies showing spiral structure in the midUV (§ 3.4), grand-design spirals ( $\$ 3.5)$, a spiral with an inner ring ( $\S 3.6)$, and edge-on spiral galaxies ( $\S 3.7)$. We discuss the late-type and dwarf spiral galaxies in $\S 3.8$ and the Magellanic irregulars in $\S 3.9$. We finish with the peculiar galaxies and merging/interacting systems $(\S 3.10)$.

Although they are not part of the selected sample, other galaxies that appear within the WFPC2 FOV will be discussed below (including their types), in context with the targeted galaxies of similar morphological type. In a few cases, our HST and ground-based images show the type listed in the RC3 to be incorrect, for various reasons explained below.

Most of our Cycle 9 mid-UV galaxies, except for some of their outskirts, fit inside a single WFPC2 CCD, and so the current paper shows only a single WFPC2 CCD in most of the panels of Figures 3 and 4 . However, a few edge-on gal-

\footnotetext{
${ }^{6}$ See http://www.asu.edu/clas/hst/www/midUV.html

${ }^{7}$ See http://www.public.asu.edu/ asusco/research/uband/red1/ and http://presto.stsci.edu/apsb/doc/pep/public-proposals/8645.prop.
} 

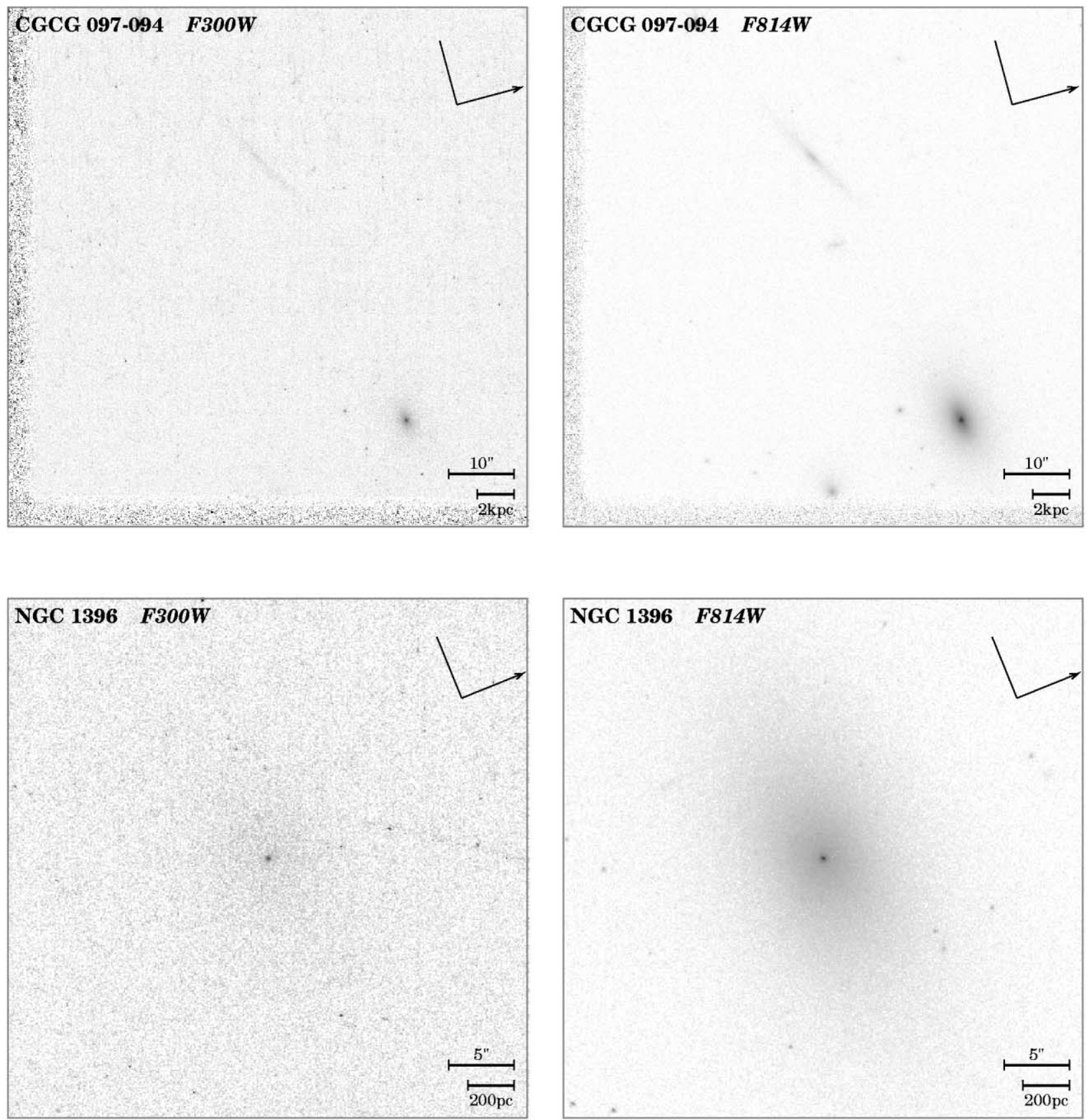

FIG. 3.-Atlas of HST WFPC2 observations of 37 nearby galaxies in the mid-UV F255W (only for the 11 galaxies in HST's CVZ) and F300W filters and in the optical $I$-band $(\mathrm{F} 814 \mathrm{~W})$ filter. The galaxies are sorted in the atlas approximately according to their observed morphological type following the discussion of the individual objects in $\S 3$. For the convenience of the reader, Table 2 cross-references the available galaxy data with the atlas figure numbering. Each of the gray-scale renditions is displayed using a logarithmic stretch, and the image scale and orientation on the sky are indicated. For some galaxies we will present images at two different image scales and stretches, in order to emphasize small-scale features discussed in the text (see $\S 3$ ). We also present images for some serendipitously observed galaxies. 3.01. CGCG 097-094 (top): WFPC2 F300W and F814W. Serendipitous observation of an early-type galaxy. Also visible are an edge-on spiral galaxy and a face-on disk galaxy (both anonymous). 3.02. NGC 1396 (bottom): WFPC2 F300W and F814W.

axies are too large to fit on a single WFPC2 CCD. For these galaxies, the relevant parts of a full WFPC2 CCD mosaic are therefore shown instead in the current paper (see UGC 10043 in Fig. 3.16). Since the number of pixels we can display in this manner on one journal page does not do full justice to the high quality of the WFPC2 data, we will display in a sequel paper (S. C. Odewahn et al. 2003, in preparation) multicolor WFPC2 mosaics in full-page size for the two remaining large edge-on galaxies (i.e., ESO 033-G022 in Fig. 3.19 and IC 4394 in Fig. 3.20) and possibly for a few other large objects, as needed. This sequel paper will dither all WFPC 2 mosaics for our sample of 37 objects and do a detailed sky subtraction across the CCDs, which is needed for reliable determination of the mosaic-wide multicolor light profiles, as well as the subsequent quantitative analysis. This paper will thus show a few additional images not shown in the current paper.

The HST images, in general, have a very high dynamic range. This makes it difficult to properly display the full range of structures within a galaxy. Each of the gray-scale 

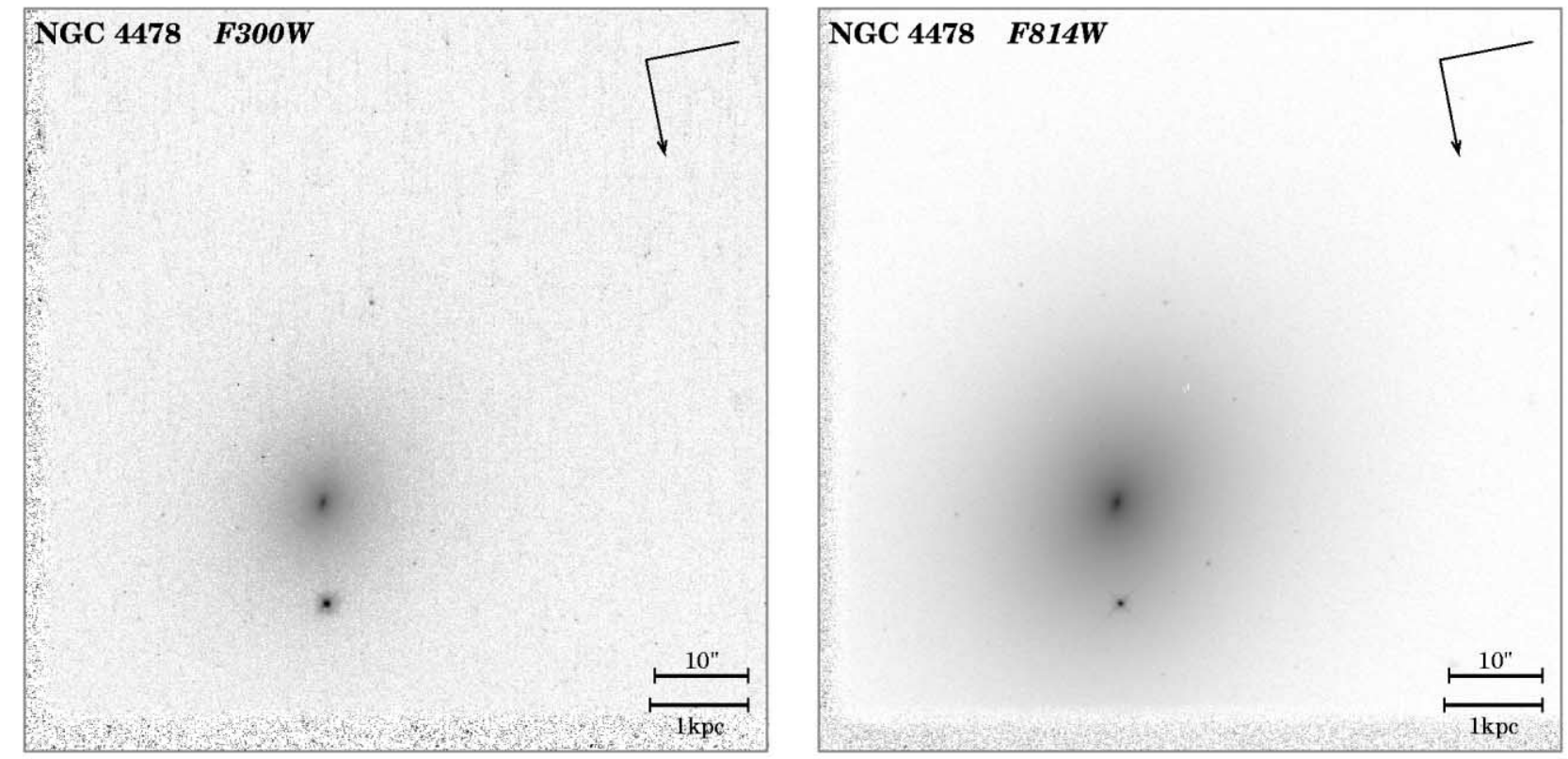

FIG. 3.-3.03. NGC 4478: WFPC2 F300W (left) and F814W (right).

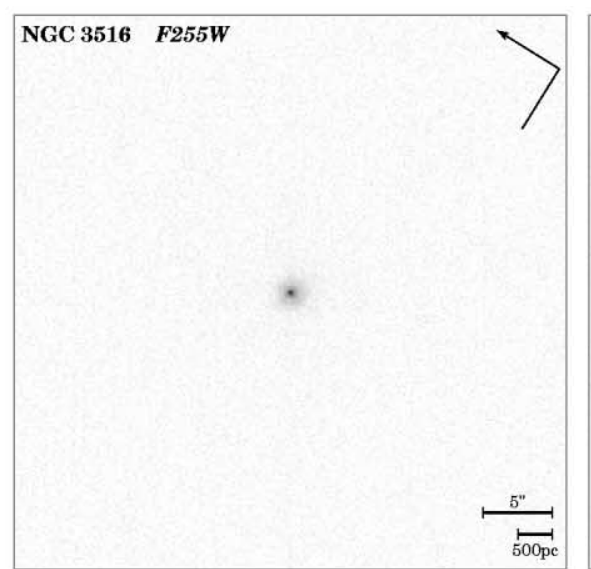

NGC $3516 \quad F 300 W$

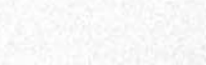

UGC 03426 (Mrk 3) F255W
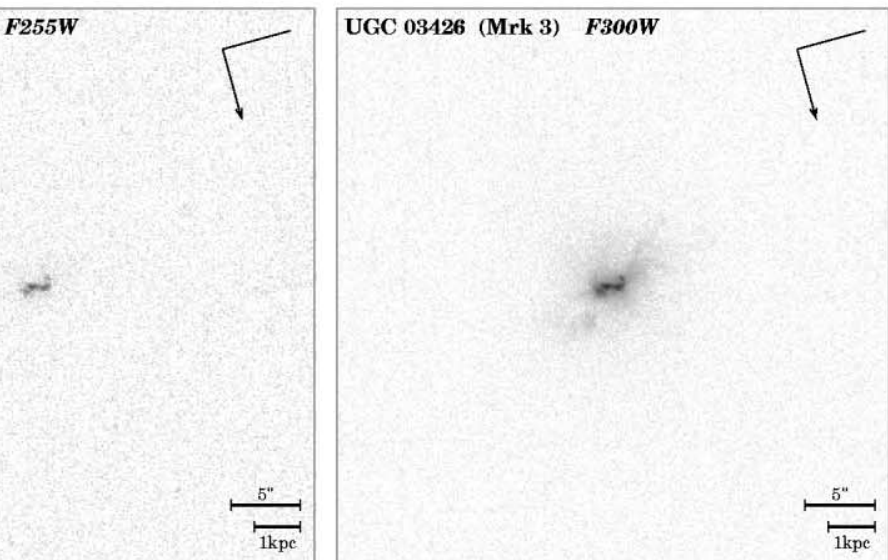

NGC 3516 F814W
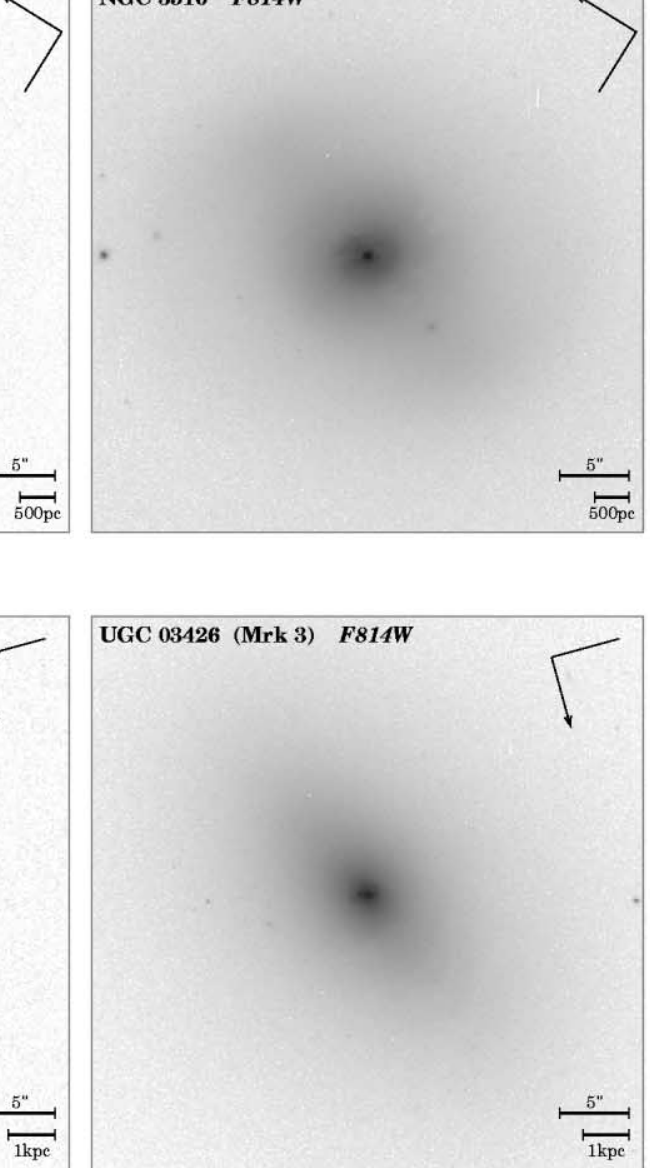

FIG. 3.-3.04. NGC 3516 (top): WFPC2 F255W, F300W, and F814W. 3.05. UGC 03426 (bottom): WFPC2 F255W, F300W, and F814W. 

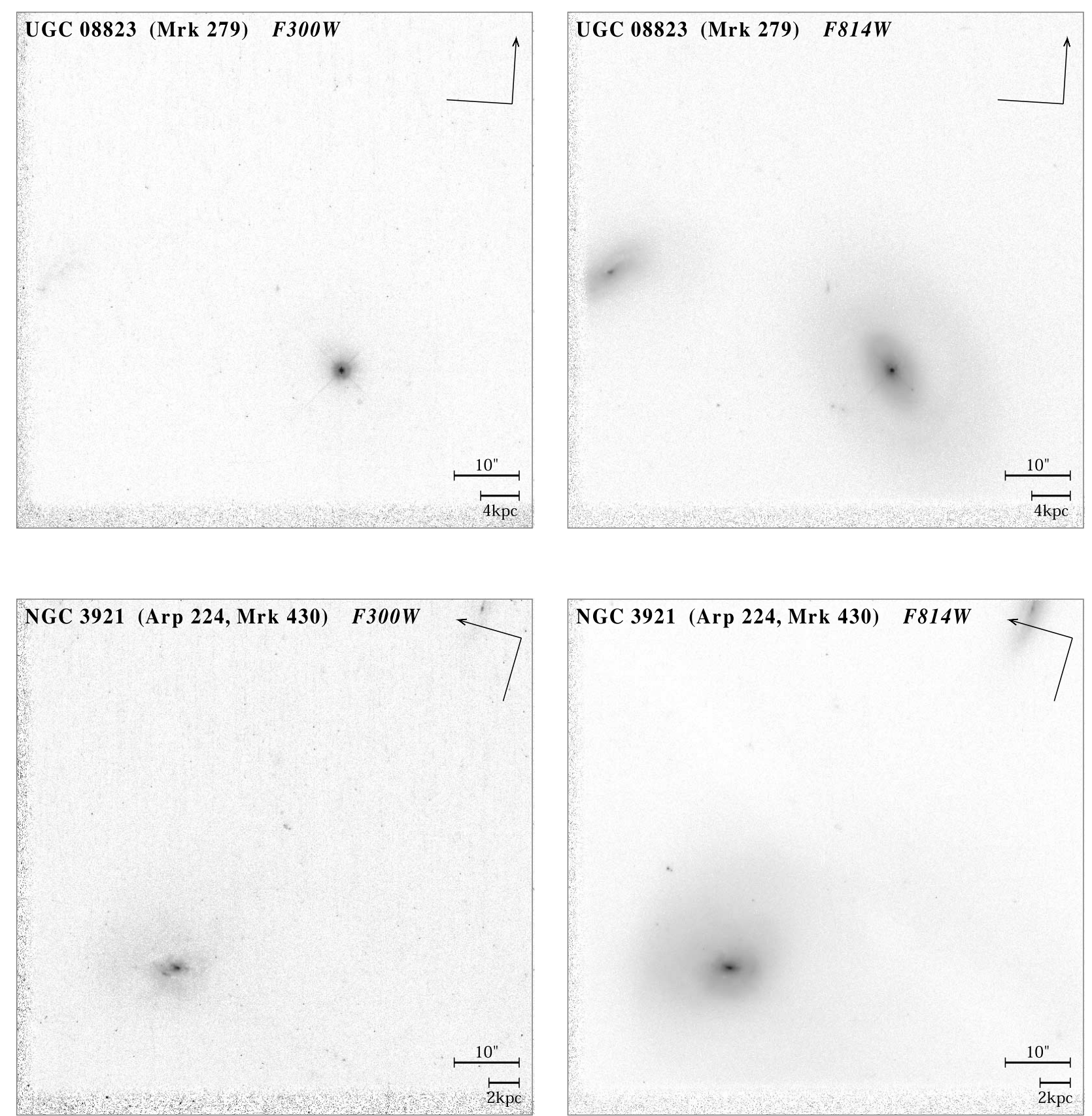

FIG. 3.-3.06. UGC 08823 (top): WFPC2 F300W and F814W. 3.07. NGC 3921 (bottom): WFPC2 F300W and F814W.

renditions in the atlas is displayed using an inverse logarithmic stretch with lower and upper display limits approximately equal to the sky background (white) and the 3 times the maximum data value in the galaxies' brightest parts (black). This compromise allows one to discern individual high-SB structures within a galaxy while still being able to see much of the faint outer parts within a galaxy. The two unexposed edges of each WFPC2 image allow one to trace the total $80^{\prime \prime} \times 80^{\prime \prime}\left(75^{\prime \prime} \times 75^{\prime \prime}\right.$ useful) FOV of a WFC CCD. When a galaxy is much smaller than the WFC FOV, or when there is too much morphological information contained within a single WFPC2 CCD, we (also) present the relevant portion of that $\mathrm{CCD}$ frame, but enlarged by a factor of 2 or 4 (i.e., a $37^{\prime \prime} \times 37^{\prime \prime}$ or $19^{\prime \prime} \times 19^{\prime \prime} \mathrm{FOV}$ ).
The ground-based images (in Fig. 4) tend to have a much smaller dynamic range (because of the much higher sky background and lower resolution). Nonetheless, we use a similarly defined stretch and display limits. Often, the extent of a galaxy becomes more obvious in these ground-based images than in the mid-UV images. In Figures 4.01-4.38 all images are displayed on the same angular scale and at the same orientation, with north up and east to the left.

\subsection{Mid-UV-faint Early-Type Galaxies}

3.01. CGCG 097-094 (T=-5; E).-This is an earlytype galaxy in A1367 that is faint in the mid-UV compared to F814W. CGCG 097-094 was observed in the field of pri- 

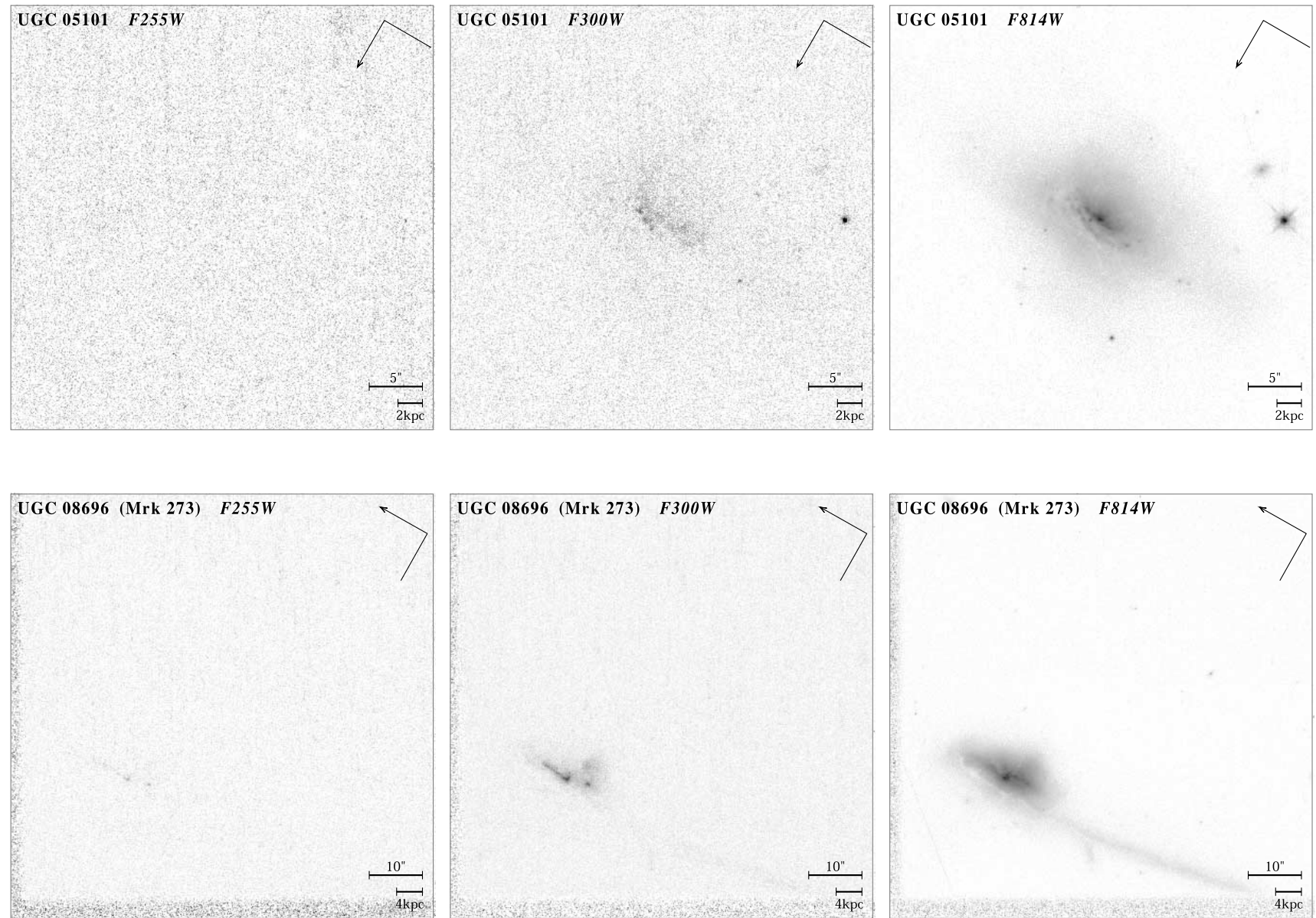

FIG. 3.-3.08. UGC 05101 (top): WFPC2 F255W, F300W, and F814W. 3.09. UGC 08696 (bottom): WFPC2 F255W, F300W, and F814W.

mary target $\mathrm{MCG}+03-30-071$. Other cluster members are also visible (Figs. 3.01 and 4.01): the edge-on galaxy is still visible in F300W, suggesting that, despite its dust content, it still transmits some light at $2930 \AA$. Most of this UV light may come just from the near edge of the disk (the side facing us). MCG +03-30-071 itself is discussed in $\S 3.8$, and edge-on galaxies are discussed further in $\S 3.7$.

The vertical image flaws (faint streaks) in the F300W image are due to WFPC2 charge transfer efficiency (CTE) effects, the severity of which has significantly increased over the past years. They are visible in the high-contrast stretches that were needed to show the faintermid-UV features of this and other objects in the atlas.

3.02. NGC $1396(\mathrm{~T}=-3$; $\mathrm{S} 0-)$.- $-\mathrm{This}$ is a lenticular galaxy that is faint in the mid-UV compared to F $814 \mathrm{~W}$, indicative of an old stellar population. It has a weak nuclear point source. NGC 1396 is not detected at $150 \mathrm{~nm}$ in the UIT farUV image of its bright neighbor NGC 1399 and is at best barely visible in the $250 \mathrm{~nm}$ UIT image. We do not have ground-based images as yet for this galaxy, and so we substitute SERC IIIaJ and POSS II IIIaF plate scans for the $B$ and $R$ images, respectively, in Figure 4.02.

3.03. NGC $4478(\mathrm{~T}=-5$; $\mathrm{E})$.- This is an elliptical galaxy that is faint in the mid-UV compared to F814W, although comparatively not as faint in F300W as some of the other early-type galaxies discussed. NGC 4478 is one of the giant elliptical galaxies in the Virgo Cluster, many of which have a UV upturn in their far-UV spectrum (Burstein et al. 1988).
This UV upturn population is expected to be mostly visible below $2000 \AA$ and contributes less than $3 \%-30 \%$ and in most cases less than $10 \%$ of the total light at $2930 \AA$ (see Figs. 6 and 7 of Burstein et al. 1988). Another point source is seen just below the nucleus. It is very bright at $\mathrm{F} 300 \mathrm{~W}$ and in the UIT $250 \mathrm{~nm}$ image and is likely a blue foreground star.

\subsection{Early-Type Galaxies with Significant AGN Emission in the Mid-UV}

3.04. NGC $3516(\mathrm{~T}=-2$; $\mathrm{S} 0)$. - NGC 3516 is a wellknown classical Seyfert 1 galaxy (Seyfert 1943; Khachikian 1974; Keel \& Weedman 1978; Kent 1985; Filippenko 1985) that becomes essentially a point source in the HST mid-UV images. The AGN-dominated F300W image shows a strong central point source exhibiting diffraction spikes. A faint extension is seen in this filter, but not in F255W. The faint fuzz seen around the central point source in the $150 \mathrm{~nm}$ UIT image is likely due in a significant part to the PSF caused by the far-UV optics plus photographic film of the UIT camera. The ground-based and F814W images show a faint bar with a position angle P.A. $\simeq-17^{\circ}$. NGC 3516 has a companion galaxy with small velocity difference (Keel 1996a, 1996b). It is also a well-known radio and X-ray source (e.g., Taylor et al. 1996; White \& Becker 1992; Laurent-Muehleisen et al. 1997; Perlman et al. 1998; Radecke 1997) and is a low-luminosity AGN with the jet pointing close to the line of sight to the observer. Quantitative PSF fits of all these 

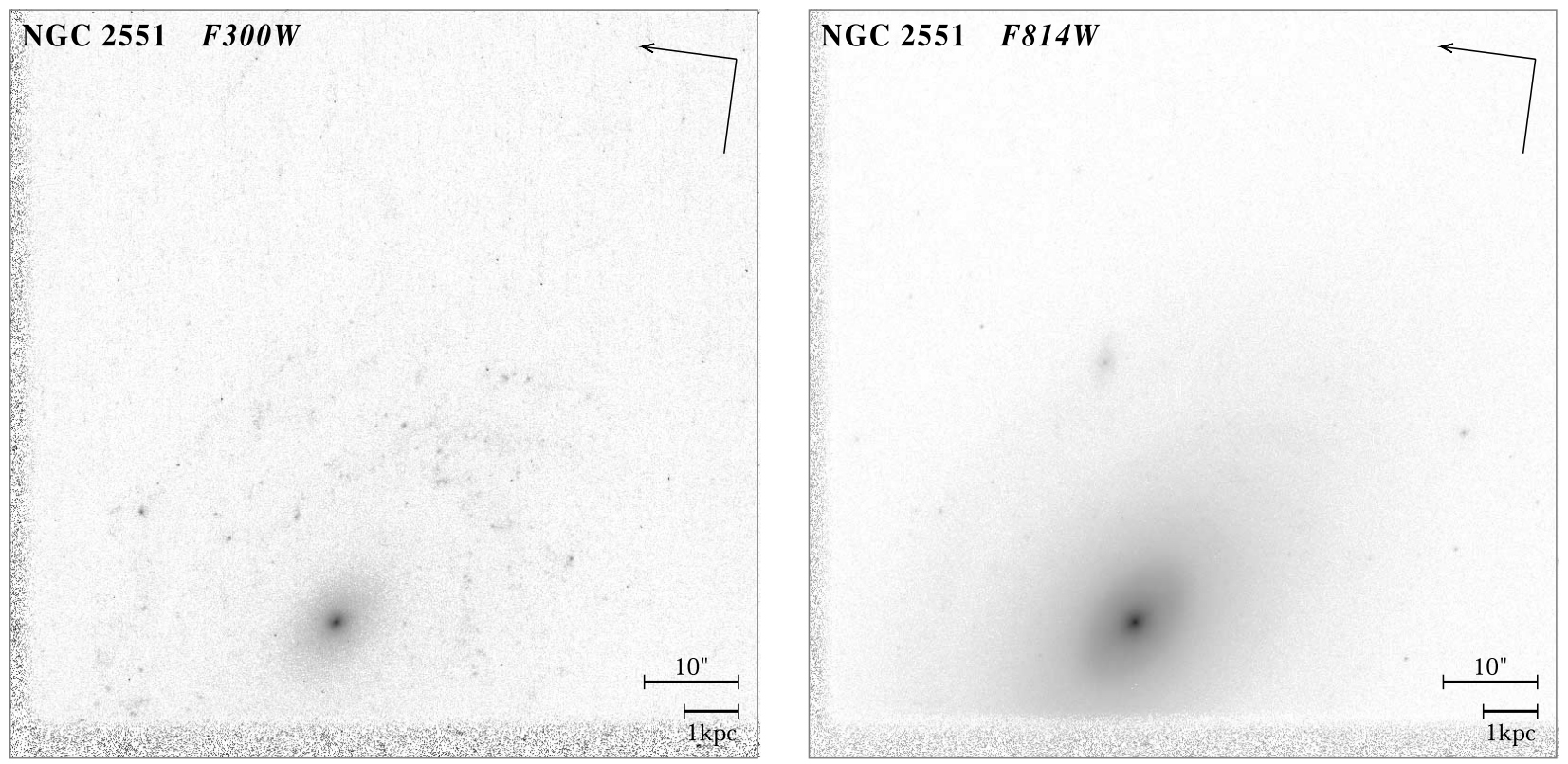

FIG. 3.-3.10. NGC 2551: WFPC2 F300W (left) and F814W (right).

AGN-dominated objects will be given by S. C. Odewahn et al. (2003, in preparation), in conjunction with the $H S T$ plus ground-based light profiles for all of our Cycle 9 sample.

3.05. UGC 03426 (a.k.a. Mrk 3; $\mathrm{T}=-2$; S0). - This is an early-type Seyfert 2 galaxy with a blue, nuclear " disklike" feature that may be associated with a bipolar outflow (Ruiz et al. 2001) rather than a nuclear star-forming disk. The feature is oriented roughly perpendicular to the galaxy major axis in F814W and is visible in both F300W and F255W. Note the apparent change in galaxy position angle blueward of the $B$ filter in Figure 4.05 .

3.06. UGC 08823 (a.k.a. Mrk 279; T $=-2$; $\mathrm{S} 0$ ). - This is an early-type galaxy that becomes essentially a point source in the mid-UV. Note that almost no extended light is seen in F300W compared to F814W. It appears to be interacting with its neighbor MCG +12-13-024, with which it forms a physical pair (Keel 1996a). It is a well-known Seyfert 1 galaxy (Khachikian 1974; Osterbrock 1977). For a highresolution optical study of this object we refer the reader to Knapen, Shlosman, \& Peletier (2000).

\subsection{Early-Type Galaxies with Evidence for Recent Merging or Strong Interaction}

The next three systems include morphologically peculiar objects with single stellar systems. We discuss these objects here, at the end of the sequence of early types, because they will likely evolve into early types on relatively short timescales ( $\lesssim 1$ Gyr; see Barnes \& Hernquist 1992) and likely already have developed $r^{1 / 4}$ profiles in their inner parts (e.g., van Albada 1982; Windhorst et al. 1994b, 1998a). Earlier stage interactions, in which the two galaxies are still separated, are discussed at the end of the sequence (§3.10), after the spirals and irregulars, since at high redshift such objects are more likely seen as interacting galaxies or peculiars.

3.07. NGC 3921 (a.k.a. Arp 224 (Arp 1969), Mrk 430, UGC 08823; T = 0; S0a).-NGC 3921 is a disturbed earlytype galaxy with a blue nuclear region that is visible in
F300W. This object is a well-studied merger remnant (Schweizer 1996; Yun \& Hibbard 2001) and was probably produced by the merger of a gas-rich and gas-poor progenitor (Hibbard \& van Gorkom 1996; Schweizer et al. 1996). It lies near the end of the Toomre sequence of ongoing mergers (Toomre 1977) and represents the final stage of merging, where there is a single nucleus but multiple tidal features. It will likely evolve into an elliptical galaxy (Schweizer 1996; Hibbard \& Yun 1999b). The ground-based images in Figure 4.07 show the fainter debris beyond the nuclear regions, including remaining tidal features. The near-IR images show a "wake" to the north of the bulge and possibly also a tidal feature from the same merger.

3.08. UGC $05101(\mathrm{~T}=11$; Pec $)$.- - This object is very faint in $\mathrm{F} 300 \mathrm{~W}$ and is not detected in F255W. The F814W images show that it has an inclined dusty disk. This object is an ultraluminous infrared galaxy (ULIRG) with $L_{\mathrm{IR}} \gtrsim 10^{12} L_{\odot}$ (Sanders et al. 1988). Like NGC 3921, it appears to be a late-stage merger remnant, with a single nucleus (Scoville et al. 2000) and multiple extended tidal features seen at larger scales in the ground-based images, including a tidal tail and a loop or polar ringlike structure (Sanders et al. 1988; Surace, Sanders, \& Evans 2000). The latter authors explain the large-scale morphology as resulting from a "plunging " collision at nonzero impact parameter of two highly inclined disks.

Structures like these are typical for a merger in progress. The tails are thought to evolve on timescales of greater than 1 Gyr, while, once the halos overlap, the galaxy nuclei evolve on much shorter timescales (of order $100 \mathrm{Myr}$ ). This results in one visible tidal tail per progenitor disk, while the other side of the disk gets pulled into a "bridge" before it disperses. A dusty disk, such as seen in UGC 05101, is a common feature of gas-rich merger remnants, e.g., NGC 2623 (Bryant \& Scoville 1999), NGC 3256 (Zepf et al. 1999), NGC 7252 (Wang, Schweizer, \& Scoville 1992; Whitmore et al. 1993), Mrk 273, and NGC 3310 (this paper). Disks have recently been reproduced in numerical simulations of merg- 

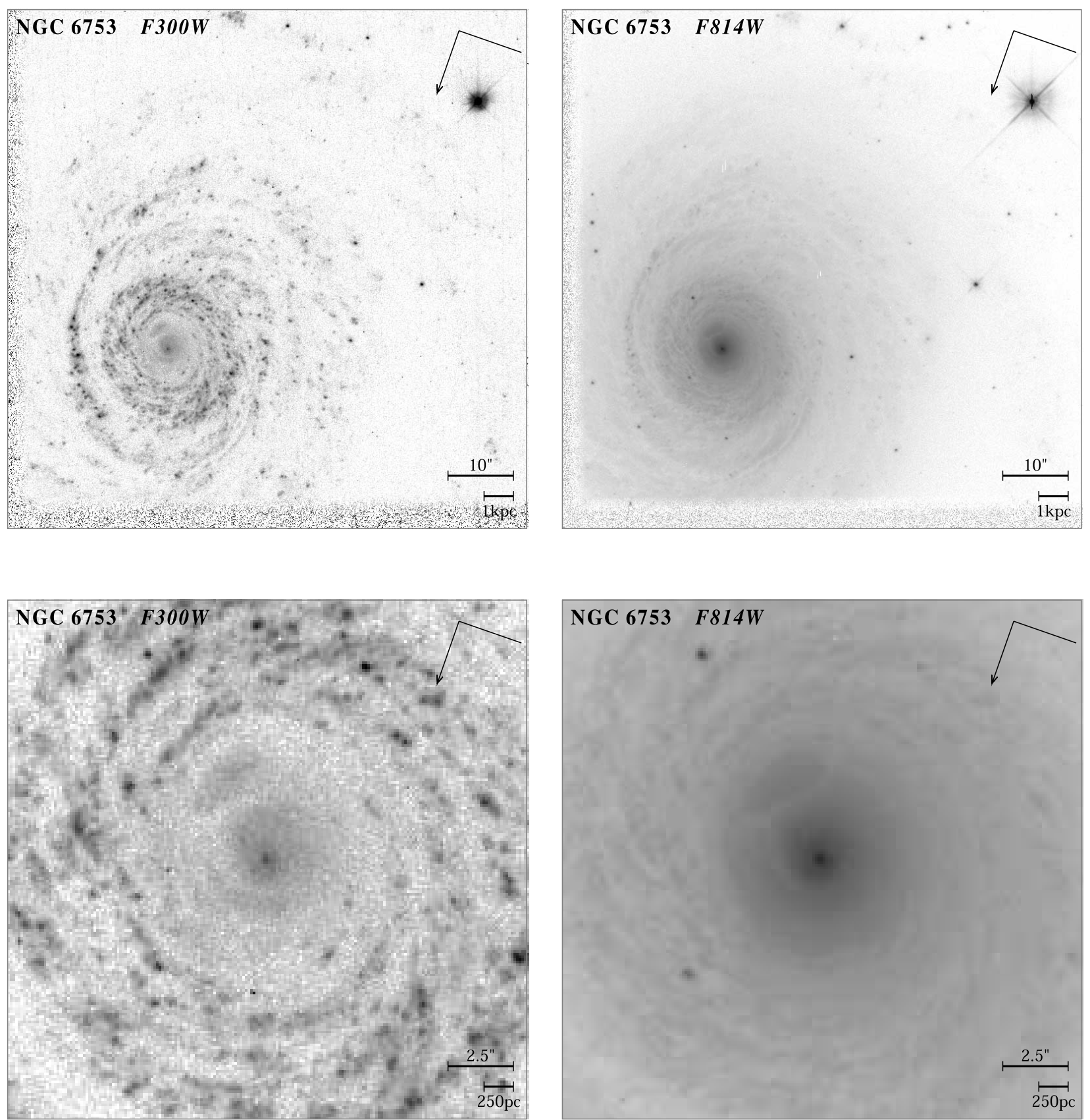

FIG. 3.-3.11a. NGC 6753 (top): WFPC2 F300W and F814W. 3.11b. NGC 6753 (bottom): nuclear portion of NGC 6753, emphasizing the dust lane crossing the inner spiral arms discussed in the text.

ing galaxies (Naab \& Burkert 2001; Barnes 2002), and hence their presence does not rule out a major merger origin, as was once thought.

3.09. UGC 08696 (a.k.a. Mrk 273, VV 851; T=11; Pec).- This object is faint in F300W and barely detected in $\mathrm{F} 255 \mathrm{~W}$. The F300W morphology is very similar to the $U^{\prime}$ morphology presented in Surace \& Sanders (2000). It appears to have a dusty disk, visible on one side of the nucleus in $\mathrm{F} 814 \mathrm{~W}$. It is a merger product, as attested to by the tidal tail evident in F814W and in the ground-based images (Fig. 4.09). The horizontal streak in the groundbased $R$-band image is due to a bright star outside the FOV. Like UGC 05101, this system is a well-known and well- studied ULIRG. But unlike the previous two systems, radio imaging and near-IR imaging reveal two distinct nuclei separated by 1" (Majewski et al. 1993; Knapen et al. 1997; Scoville et al. 2000). One of the near-IR and radio nuclei coincides with the brightest peak in the F300W image, but the second near-IR nucleus is obscured, even at F814W. The southern peak in the F300W image has no optical or near-IR counterpart and is probably a very young star cluster (Surace \& Sanders 2000). CO line mapping reveals the presence of two orthogonal kinematic components (Yun \& Scoville 1995; Downes \& Solomon 1998), suggesting a highly inclined encounter between two gas-rich systems. 

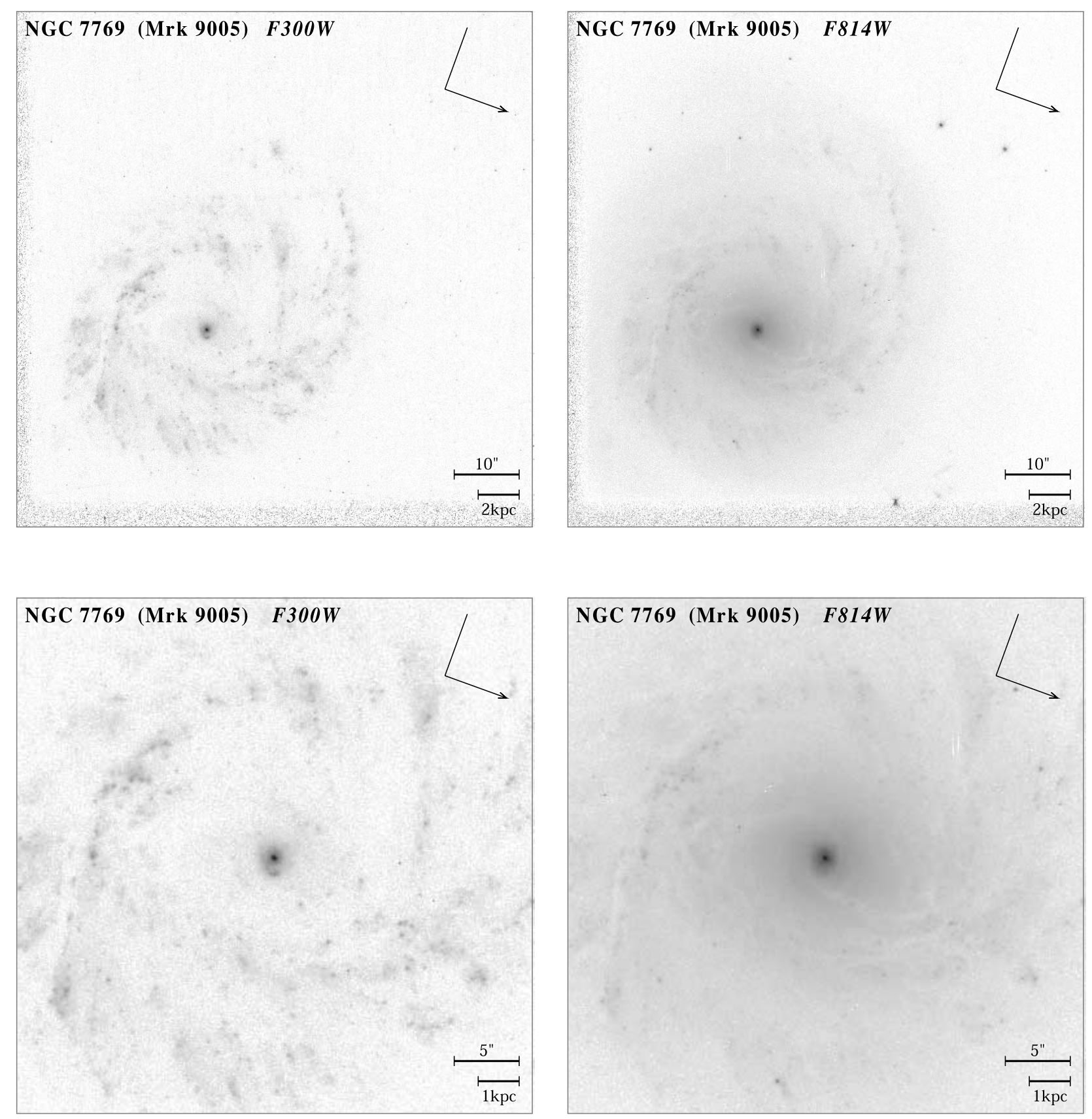

FIG. 3.-3.12a. NGC 7769 (top): WFPC2 F300W and F814W. 3.12b. NGC 7769 (bottom): central portion of NGC 7769, emphasizing the dust lanes crossing the spiral arms and the dust lane crossing its small bulge.

\subsection{Early-Type Galaxies Displaying Spiral Structure in the Mid-UV}

3.10. NGC $2551(\mathrm{~T}=0.2$; $\mathrm{S} 0 \mathrm{a})$.- $-\mathrm{This}$ is an early-type spiral galaxy, whose spiral structure is much more pronounced in F300W than in F814W. In the optical/red, the galaxy would be classified as an S0a, in the mid-UV as an Scd, so its morphological $K$-correction is significant. NGC 2551's bulge is faint in the mid-UV compared to F814W, although it seems bluer than that of other early-type spirals. The background galaxy seen in F814W does not appear in F300W, but this does not necessarily mean that the disk of NGC 2551 is very dusty at this location: the background galaxy may just be as red as some of the other field galaxies seen in the background of our other images (we chose the exposure times in F300W and F814W to provide relatively high $\mathrm{S} / \mathrm{N}$ for nearby galaxies, not for higher redshift ones). Figure 4.10 shows that NGC 2551 was also detected with UIT at $250 \mathrm{~nm}$, but probably not at $150 \mathrm{~nm}$. The UIT resolution (FWHM $\sim 3^{\prime \prime}$ ) is much lower than HST's, but on large scales the UIT $250 \mathrm{~nm}$ morphology is comparable to our HST F300W image.

\subsection{Grand-Design Spiral Galaxies}

3.11. NGC $6753(\mathrm{~T}=3$; $\mathrm{Sb})$.- This is a grand-design spiral galaxy with very pronounced spiral structure in both F814W and F300W. Dust lanes are visible crossing the spiral arms in $\mathrm{F} 814 \mathrm{~W}$, corresponding to regions of reduced or no light in F300W. A detailed discussion of the HST images 

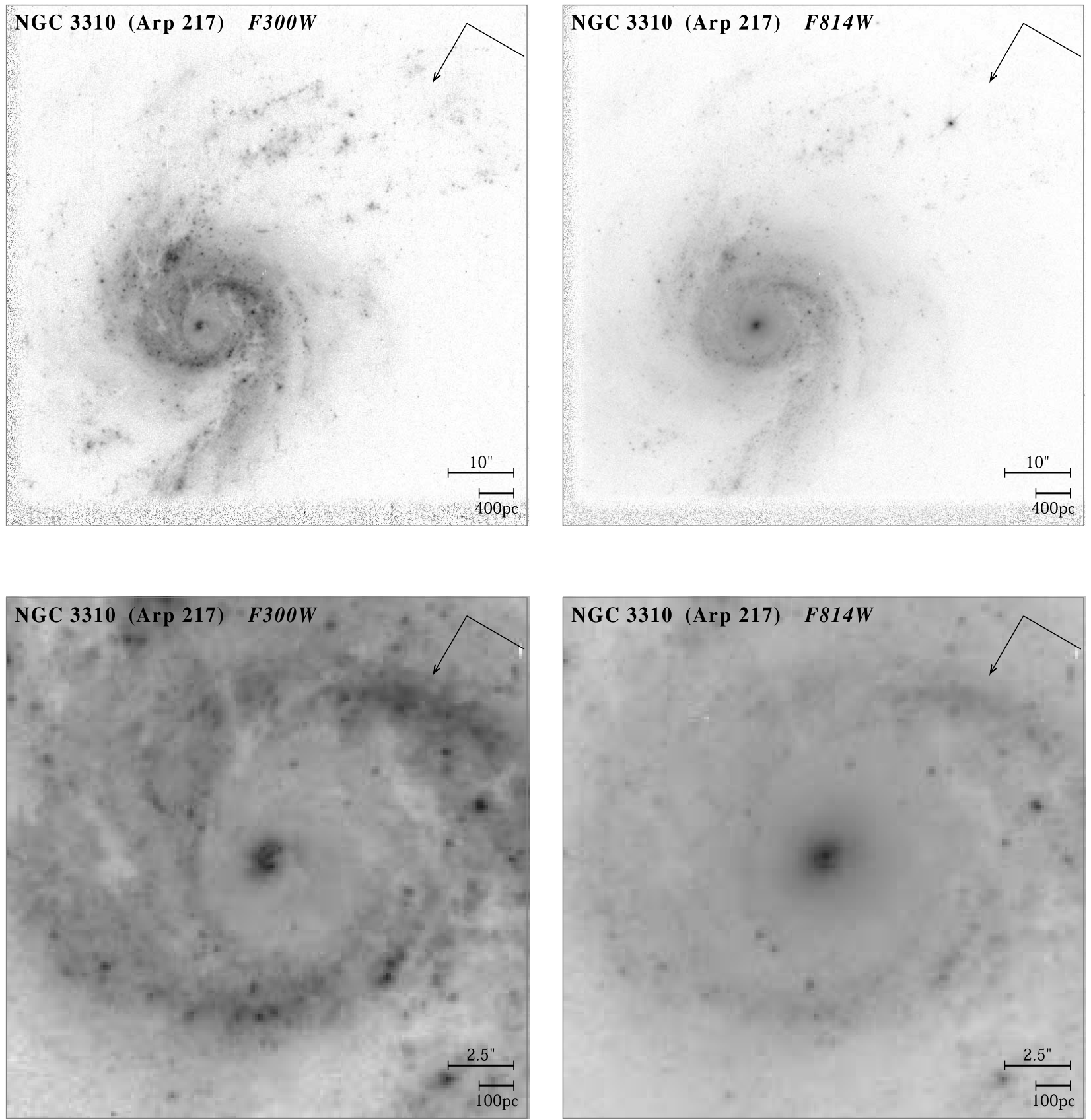

FIG. 3.-3.13a. NGC 3310 (top): WFPC2 F300W and F814W. 3.13b. NGC 3310 (bottom): nuclear portion of NGC 3310, emphasizing the spiral arm and nucleus crossing dust lanes.

of this object is given by P. B. Eskridge et al. (2002, in preparation). An enlargement of Figure 3.11 $a$ is shown in Figure $3.11 b$. In addition to dust lanes crossing the spiral arms in a regular pattern and with a fairly constant pitch angle, there is a large dust lane crossing the inner spiral arms under a significantly different pitch angle, best seen in the F814W image. This is likely a foreground trail of dust (see also discussion of NGC 5278/79 in $\S 3.10$ ). Figure 4.11 shows that the morphological $K$-correction is modest from F300W through the $I$ band, although in the near-IR the object appears to be of earlier type in ground-based seeing.

3.12. NGC 7769 (a.k.a. Mrk 9005; T = 3; Sb). - This is a grand-design spiral galaxy with very significant spiral structure in $\mathrm{F} 300 \mathrm{~W}$, which is more pronounced than that seen in
F814W, suggesting active star formation. In the optical/ red, the galaxy would be classified as an Sb, in the mid-UV as an Scd, so its morphological $K$-correction is significant. The enlargement in Figure 3.12b shows dust lanes crossing the spiral arms in $\mathrm{F} 814 \mathrm{~W}$, corresponding to regions of reduced or no light in F300W. The object also shows a faint nuclear dust lane crossing its small bulge. NGC 7769 is interacting with SBa galaxy NGC 7771. H I mapping observations by Nordgren et al. (1997) show NGC 7771 to have an extended gaseous tail, while NGC 7769 counterrotates with respect to NGC 7771. Therefore, this is thought to be a prograde-retrograde encounter, with NGC 7769 having retrograde kinematics. Note the possible emergence of a bar in the ground-based near-IR $J$ - and $H$-band images. 

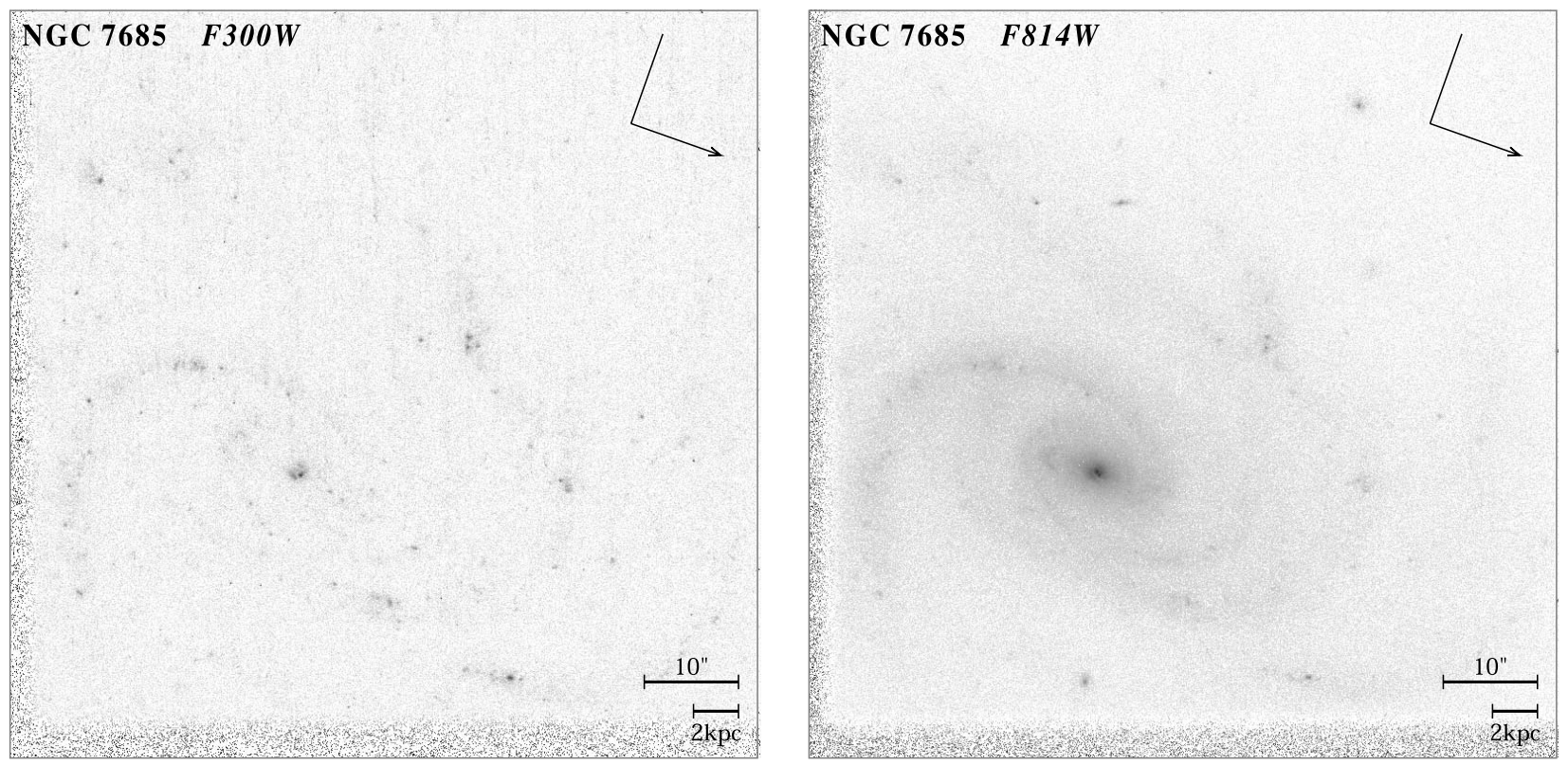

FIG. 3.-3.14. NGC 7685: WFPC2 F300W (left) and F814W (right).

3.13. NGC 3310 (a.k.a. Arp 217, VV 356; T = 4; Sbc).This mid-type spiral galaxy is well known to harbor a substantial global starburst, likely either the remnant of a merger or a significant accretion event (Balick \& Heckman 1981; Mulder, van Driel, \& Braine 1995; Smith et al. 1996b; Kregel \& Sancisi 2001 and references therein). Intense star formation is readily visible in the nuclear ring (Conselice et al. 2000), the spiral arms, and the linear "arrow" feature (e.g., Mulder \& van Driel 1996). H i mapping of this object shows extended H I tails (Kregel \& Sancisi 2001), and it is likely that the spiral "arms" do not lie in a single plane. As a result of the global starburst, the morphology of NGC 3310 remains similar in the F300W and F814W images. Some differences in structure between the red and mid-UV result from dust features, however. The enlargement in Figure $3.13 b$ shows that the galaxy nucleus appears displaced from the line of symmetry of the inner spiral arms. Figure $3.13 b$ also shows a small nuclear dust lane crossing its small bulge, as well as dust lanes crossing the spiral arms (some at significant pitch angles) in $\mathrm{F} 814 \mathrm{~W}$, corresponding to regions of reduced or no light in F300W.

The ground-based images in Figure 4.13 show that the outer spiral arms are more complicated and appear to dissolve in the southern part at all wavelengths. The far-UV morphology of NGC 3310 at UIT spatial resolution (Smith et al. 1996b) is also shown for comparison. This is an example of a galaxy that looks rather similar in all filters from the far-UV through the red.

3.14. NGC $7685(\mathrm{~T}=5.3 ; \mathrm{Sc})$. - This is a barred late-type spiral with significant spiral structure in F300W and F814W. A small bar and nuclear bulge are visible in F814W. The bulge appears to be bisected by a small nuclear dust lane. Figure 4.14 shows that the morphological $K$-correction is modest from F300W through the $K$ band.

\subsection{Spiral Galaxies with Inner Rings}

3.15. NGC $6782(\mathrm{~T}=0.8$; Sa).-This is an early-type spiral galaxy with a spectacular ring structure in the mid-UV, visible in both F300W and F255W. An enlargement of Figure $3.15 a$ is shown in Figure 3.15b. This ring is surrounded by two faint and apparently partially dusty spiral arms as visible in F814W and an outer spiral structure and outer ring that is visible in $\mathrm{F} 300 \mathrm{~W}$ and in the optical passbands (Fig. 4.15). The inner ring appears to be driven by a small bar visible in F814W and F300W at P.A. $=-30^{\circ}$. The ground-based images in Figure 4.15 show that the outer ring encloses an outer bar, with a position angle (P.A. $\simeq 0^{\circ}$ ) that differs from that of the inner bar visible in the HST images. A detailed discussion of the HST images of this object is given by P. B. Eskridge et al. (2002, in preparation). Indepth studies of galaxies with such star-forming rings are given by Buta, Purcell, \& Crocker (1996), Buta \& Purcell (1998), and Buta et al. (1998, 1999, 2000, 2001). Figure 4.15 suggests a significant morphological $K$-correction from F255W through the $K$ band, most of which is caused by the ring, but with the bars being more prevalent at the longer wavelengths, as noticed in the ground-based near-IR study of Eskridge et al. (2000).

\subsection{Edge-on Spiral Galaxies}

Here we discuss edge-on galaxies as a single morphological class to allow easier comparison, despite the fact that their intrinsic $\mathrm{T}$ types may span most of the Hubble sequence.

3.16. UGC $10043(\mathrm{~T}=4.0$; $\mathrm{Sbc} \mathrm{pec})$.- - This is an edge-on spiral galaxy that is faint in $\mathrm{F} 300 \mathrm{~W}$, although it is still visible in this filter almost throughout the entire disk. The central bulge is relatively small in size and faint in the mid-UV, as for most of the other edge-on galaxies in our sample. The P.A. of the (major axis of the) bulge is approximately perpendicular to that of the disk, which is most clearly seen in the HST observations when compared to the ground-based images (Fig. 4.16). This may indicate a polar ring, which interpretation we hope to confirm with spectroscopy in a future paper. The F814W images show significant dust lanes, mostly in the inner parts of the edge-on disk. Com- 

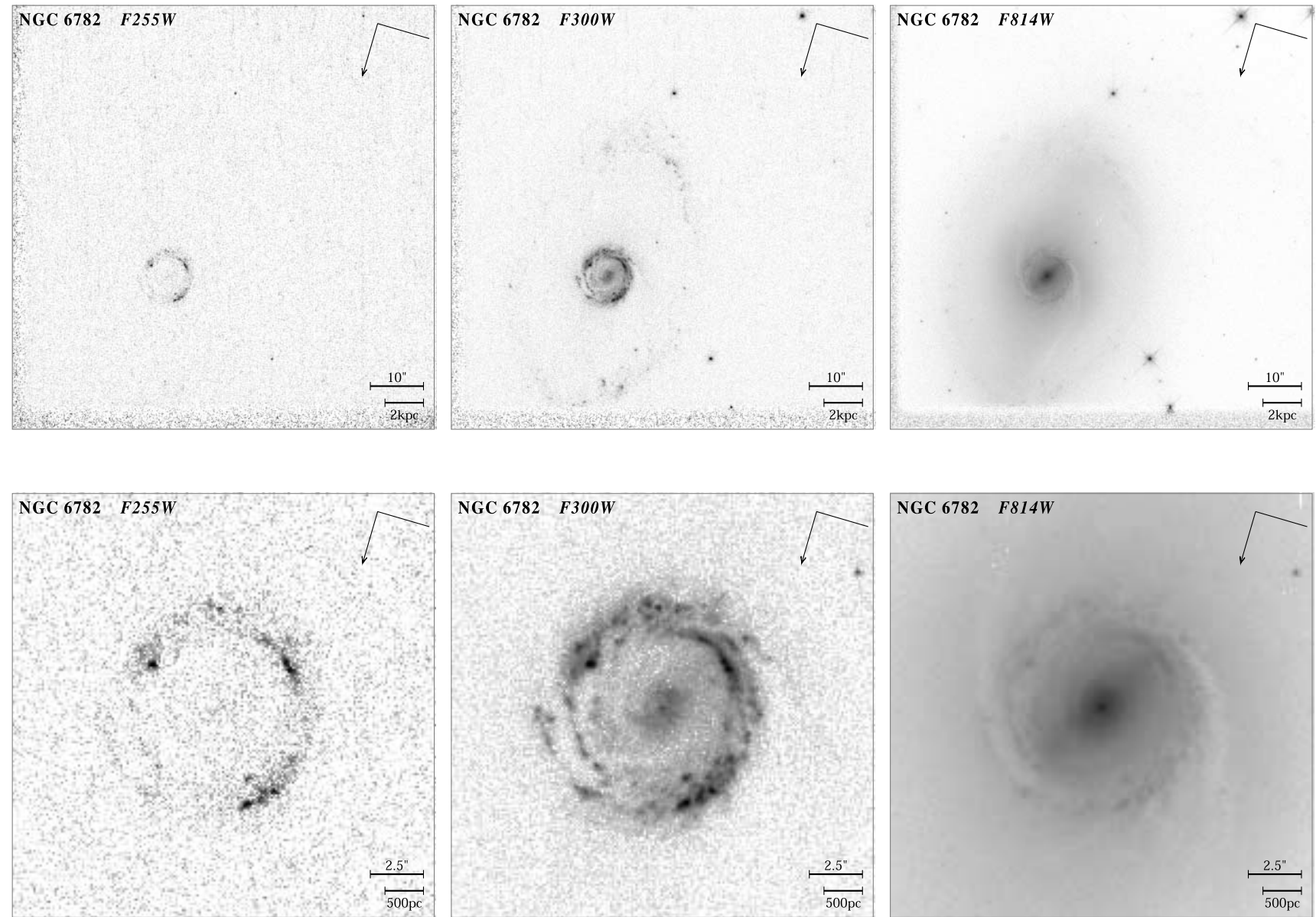

Fig. 3.-3.15a. NGC 6782 (top): WFPC2 F255W, F300W, and F814W. 3.15b. NGC 6782 (bottom): enlargement of the central portion of NGC 6782, emphasizing the spectacular ring of hot, young stars.

pared to F814W, the F300W flux shines through better in the outskirts than in the central part of the disk, and in particular, it shines through well in several bright knots. It is also possible that, instead, the knots visible in the mid-UV are in front of most of the dust.

Qualitatively, it appears that the dust extinction (if this relative dimming of the UV light is indeed due to dust extinction) decreases from the inside outward (see also Jansen et al. 1994). Examples of these edge-on objects have been studied in the optical. In the mid-UV, edge-on disk galaxies have a large range in brightness. Some are particularly bright in the UV (e.g., UGC 06697, Marcum et al. 2001; NGC 4631, Smith et al. 2001), while others are much fainter, such as some of the edge-on galaxies shown in the current paper. As pointed out by Kuchinski et al. (2001), it is as yet unclear to which degree dust attenuation affects the appearance of highly inclined galaxies at UV wavelengths. This is due to a likely complex dependence on the distribution of dust and actively star-forming regions throughout a galactic disk (Kuchinski et al. 2001). As a result of the lack of a statistically significant population of edge-on galaxies observed in mid-UV light, a detailed discussion of this effect is beyond the scope of the present paper but will be addressed in a future paper when more edge-on objects have been observed in the mid-UV.

3.17. UGC $06697(\mathrm{~T}=10$; $\mathrm{Im})$.- - This is a late-type edgeon galaxy with almost no bulge and with significant dust lanes seen by comparing F300W to F814W. Although this galaxy is bright in the UV and was observed both at 2500 and $1500 \AA$ by UIT, comparison of the F300W and F814W images shows that several regions seem to be completely obscured by dust. They appear to be dust pockets that bisect the disk. The ground-based images in Figure 4.17 show a significant warp on larger scales, which is possibly related to the two or three companion galaxies visible within the FOV. On balance, however, the overall appearance of this object is very similar from the far-UV through F814W.

3.18. IC $3949(\mathrm{~T}=-2$; $\mathrm{S} 0$ pec).-Edge-on spiral with a very faint bulge, if any, and significant dust lanes (compare the F300W and F814W images), especially in the lower (western) part of the displayed image. The plane of the dust appears to be warped and asymmetric in the western part of the image compared to the upper (eastern) part. The biggest dust pocket to the lower right of the center is also visible as a depression in the light in the ground-based $U B V R$ images (Fig. 4.18).

Four faint edge-on galaxies are seen to the left of IC 3949 in F814W (Fig. 3.18a), two of which are barely visible in F300W. It is perhaps noteworthy that all four of these highly inclined galaxies point their major axis toward the central part of IC 3949. Although this is probably a chance alignment and the edge-on galaxies are probably background galaxies, it would be interesting to test this hypothesis with further kinematic data. If these systems turn out to 

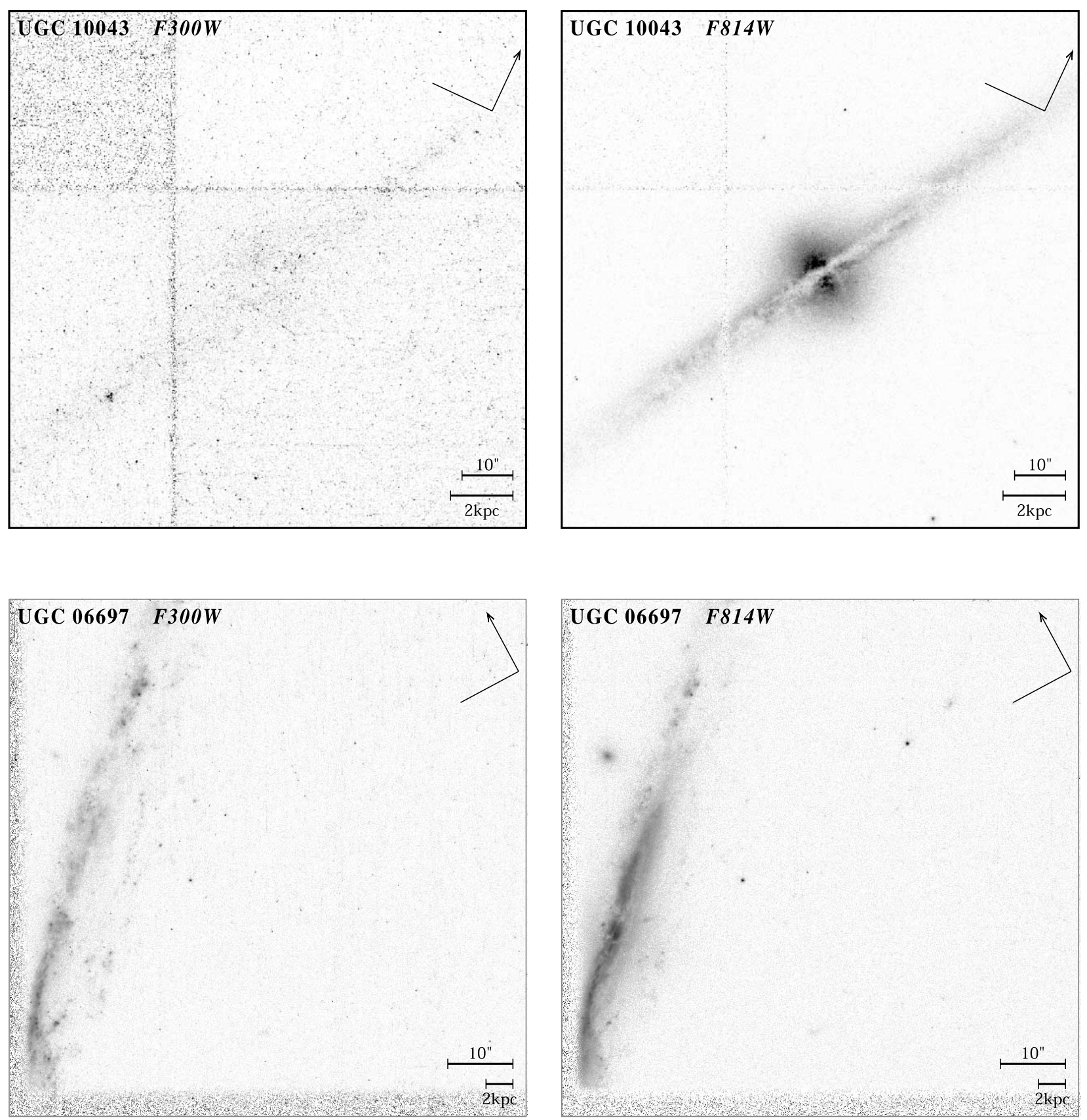

FIG. 3.-3.16. UGC 10043 (top): WFPC2 F300W and F814W. 3.17. UGC 06697 (bottom): WFPC2 F300W and F814W.

be at a similar distance as IC 3949 , they might be smaller dwarflike objects falling into IC 3949, perhaps disturbing its disk as the irregularly distributed edge-on dust lanes might suggest. Schwarzkopf \& Dettmar (2000) found that edge-on galaxies have on average $60 \%$ thicker disks and are $\sim 2$ times more likely to have warps when they have low-mass companions, which will likely soon result in minor mergers.

Figure $3.18 b$ shows other early-type objects surrounding IC 3949, which, like other early-type galaxies in our HST sample, have dim light distributions in F300W. The RC3 incorrectly classifies IC 3949 as S0 pec, which may be due to confusion with the brighter of these early-type galaxies, although this object is fainter than the edge-on galaxy. These objects were selected as field elliptical galaxies and do not show AGNs in the UV (unlike the others in our sample), although the two brightest ones show a small central disk or boxy isophotal structure.

3.19. ESO 033-G022 ( $\mathrm{T}=7$; $\mathrm{Sd})$.- - This is an edge-on late-type spiral galaxy that is faint in F300W, although it is still barely visible in this filter throughout most of the disk. There is almost no central bulge. The F814W images show a thin dust lane in the plane of the galaxy, mostly in the inner parts of the edge-on disk. Compared to F814W, the F300W flux shines through better in the outskirts than in the central part of the disk and, in particular, shines through well in several bright knots. In the ground-based images for this low Galactic latitude object from de Grijs et al. (1997; see Fig. 4.19), the bright stars (which are apparent in the F814W HST image) have been removed to allow for more accurate surface photometry. 

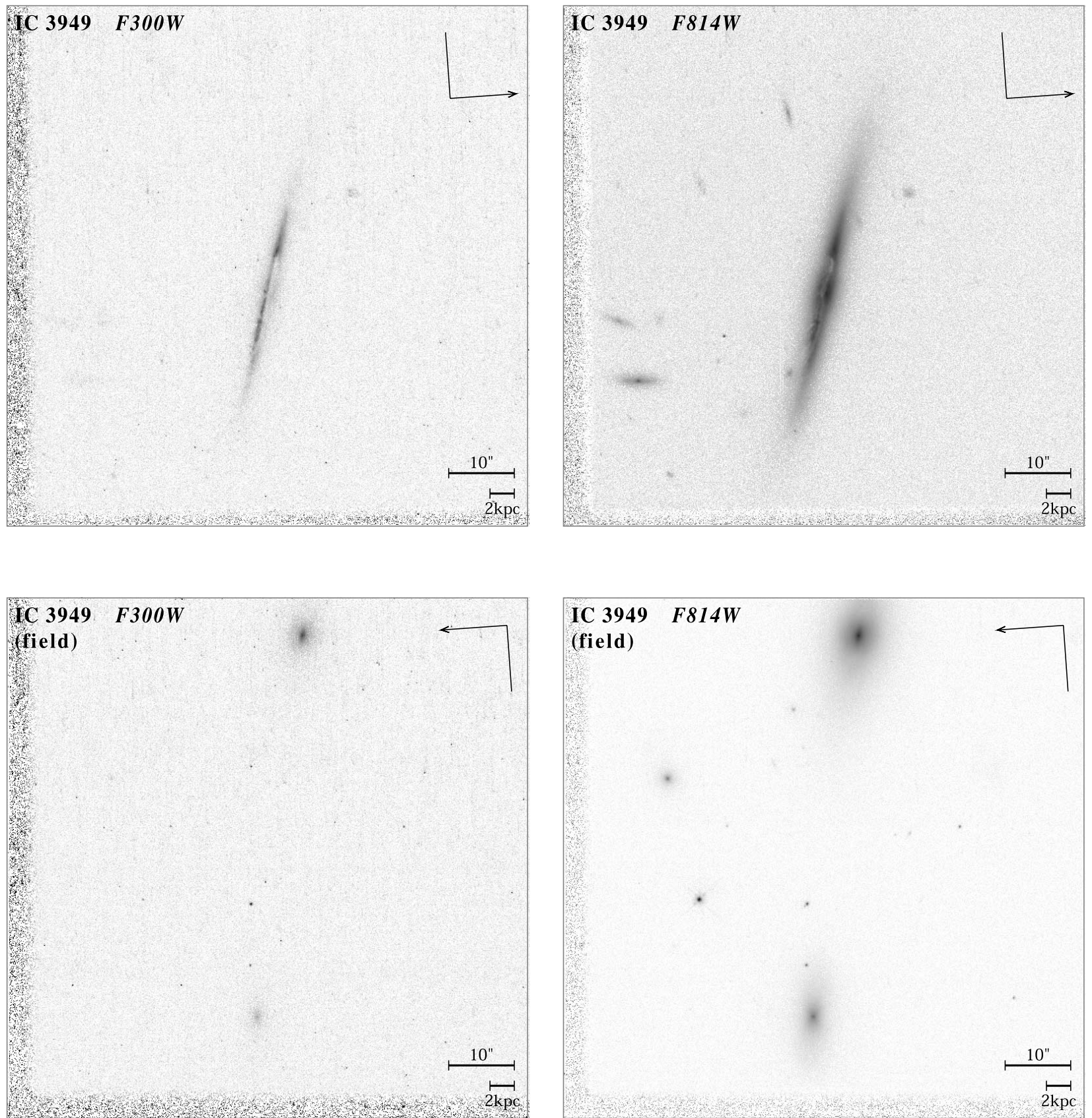

FIG. 3. - 3.18a. IC 3949 (top): WFPC2 F300W and F814W. Note the four highly inclined systems visible in F814W that have their major axes pointing toward IC 3949. 3.18b. IC 3949 (field) (bottom): several serendipitously observed early-type galaxies.

3.20. IC 4394 (a.k.a. ESO 446-G044; T =6; Scd).-This is an edge-on spiral galaxy that shows a thick dust lane in the plane of the galaxy in F814W, mostly in the inner parts of the edge-on disk. This galaxy is fainter in F300W than F814W as well, but comparatively not nearly as much as most of the other edge-on galaxies. This could be because its inclination is not as close to $90^{\circ}$ as some of the others, allowing a more unimpeded view of the bright star-forming regions located on the near side of the galactic plane. Hence, the relative bright appearance in F300W does not imply a smaller dust content than inferred in other edge-on galaxies. Almost no central bulge is visible. Compared to F $814 \mathrm{~W}$, the F300W flux shines through better in the outskirts than in the central parts of the disk and, in particular, in several bright knots, more so than seen in the other galaxies.

\subsection{Late-Type Spirals and Smaller Spirals}

3.21. MCG +03-30-071 $(\mathrm{T}=10$; $\mathrm{Im})$. - This is a small late-type spiral galaxy in A1367, displaying a four-armed spiral pattern that dissolves into individual star-forming knots in F300W. MCG +03-30-071 was detected by UIT at both 1500 and $2500 \AA$. There are several galaxies at small projected distances to this galaxy, but the smallest difference in velocity is $715 \mathrm{~km} \mathrm{~s}^{-1}$ (for the early-type neighbor $\sim 40^{\prime \prime}$ to the southwest, MCG $+03-30-067$, which is also visible in 

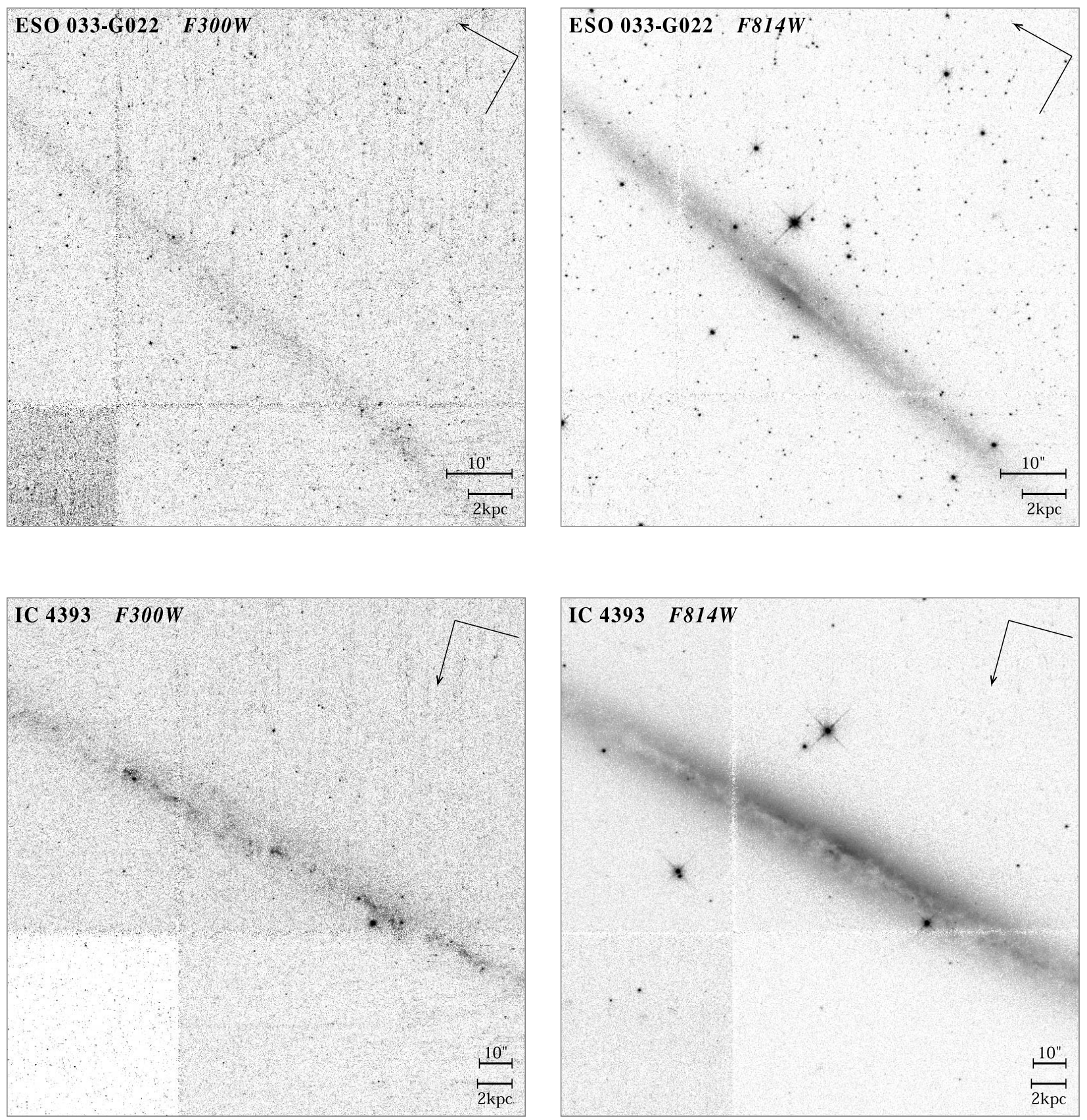

FIG. 3.-3.19. ESO 033-G022 (top): WFPC2 F300W and F814W. 3.20. IC 4393 (bottom): WFPC2 F300W and F814W.

the FOV). Comparing these two galaxies from the far-UV to $\mathrm{F} 814 \mathrm{~W}$ gives the best example of the general trends observed in this work (see the discussion in $\S 4$ ).

3.22. $\mathrm{MCG}+06-24-047(\mathrm{~T}=4$; $\mathrm{SBc})$.- $-\mathrm{This}$ is a small spiral galaxy with well-developed spiral structure that is more pronounced in $\mathrm{F} 300 \mathrm{~W}$, although still clearly visible in $\mathrm{F} 814 \mathrm{~W}$. The classification in the RC3 as $\mathrm{T}=11 / \mathrm{Pec}$ probably resulted from the bright foreground star $\sim 7^{\prime \prime}$ south of the nucleus. In the mid-UV this galaxy would be classified as $\mathrm{Sd} / \mathrm{Irr}$ rather than $\mathrm{SBc}$, so the morphological $K$-correction is nonzero. The ground-based $U B V R$ images (Fig. 4.22) show a rather modest dependence of the amplitude of the spiral structure on rest-frame wavelength, so most of the morphological $K$-correction is caused by the stellar population below the Balmer break. A small bulge is visible in
F814W. The bright star is blue (it is even marginally detected at $1500 \AA$ ), so that the F300W appearance is not significantly affected by the filters' red leak.

3.23. UGC 05028/29 (a.k.a. Arp 300, VV 106; T = $3+9$; $\mathrm{Sbc}+\mathrm{SBdm} / \mathrm{Pec}$ ). - Figure 3.23a shows the small late-type/ peculiar spiral galaxy UGC 05028 (Mrk 111) of the pair Arp 300 (see Fig. 4.23). It has spiral structure that is more pronounced in F300W although still clearly visible in F814W. Ground-based seeing hides most of the irregular nature of this object. No bulge is visible in F814W, although a small central barlike structure is visible in both filters. The brightest "knot" in F300W and particularly in F814W, south of the center, is well resolved. Although reddening by dust plays a significant role, this " knot" may be the remnant of another small late-type galaxy that is merging with UGC 

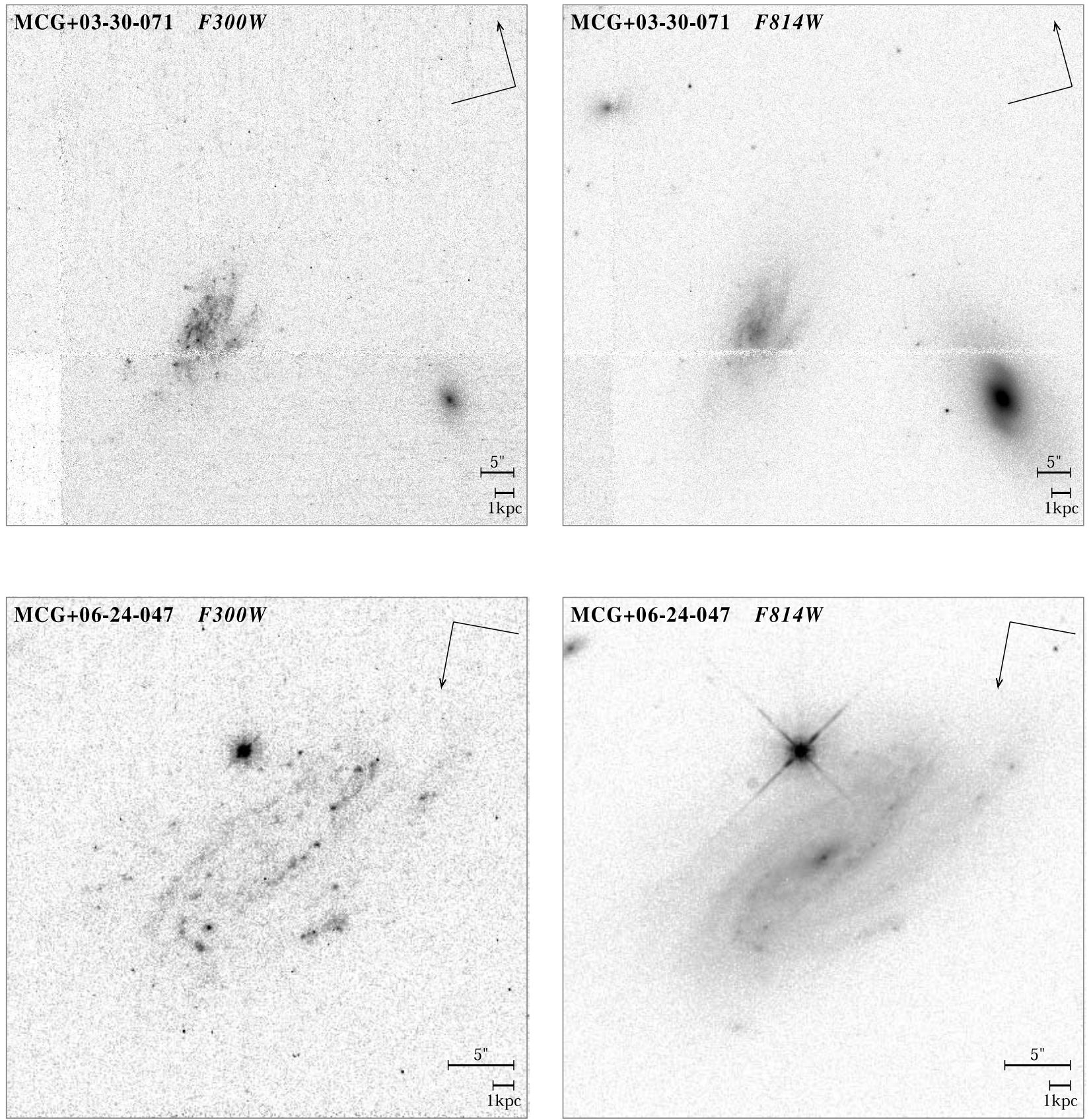

FIG. 3.-3.21. MCG +03-30-071 (top): WFPC2 F300W and F814W. Also visible is neighboring early-type galaxy MCG +03-30-067. Comparison of the F300W and F814W images of these two galaxies gives the best example of the general trends seen in the present work. 3.22. MCG +06-24-047 (bottom): WFPC2 F300W and F814W. The bright point source is a galactic (foreground) star.

05028, as its color is similar to that of the small bulge of UGC 05028's larger companion UGC 05029. If so, this knot and the bar will eventually merge and form a central bulge. As presently observed UGC 05028 is extremely asymmetric.

Figure $3.23 b$ shows the second member of the Arp 300 system, Sbc spiral galaxy UGC 05029, which is a physical companion to UGC 05028 . The spiral structure in this galaxy is more pronounced in $\mathrm{F} 300 \mathrm{~W}$, although it is clearly also visible in $\mathrm{F} 814 \mathrm{~W}$, like for some of the other early- to mid-type spirals discussed above (e.g., NGC 2551). Most of the blue $\mathrm{OB}$ associations are located on the side facing UGC 05028. Another edge-on field galaxy is seen in Figure. 4.23 but is too faint to be resolved into star-forming regions. The five rather red objects between this edge-on galaxy and the bright mid-type spiral are likely a group of background galaxies: they are not seen in F300W at all (see the discussion of other background objects above).

3.24. NGC 3860B (a.k.a. CGCG 97-114; $\mathrm{T}=10$; Im). This is a small late-type spiral galaxy with spiral structure that is more pronounced in $\mathrm{F} 300 \mathrm{~W}$, although it is still visible in F814W. Its rather late classification probably resulted from the fact that the spiral structure is only barely visible in the ground-based images. The UIT, HST, and groundbased images show only a modest dependence of morphology on rest-frame wavelength. A very small bulge is visible in F814W (Fig. 3.24). 

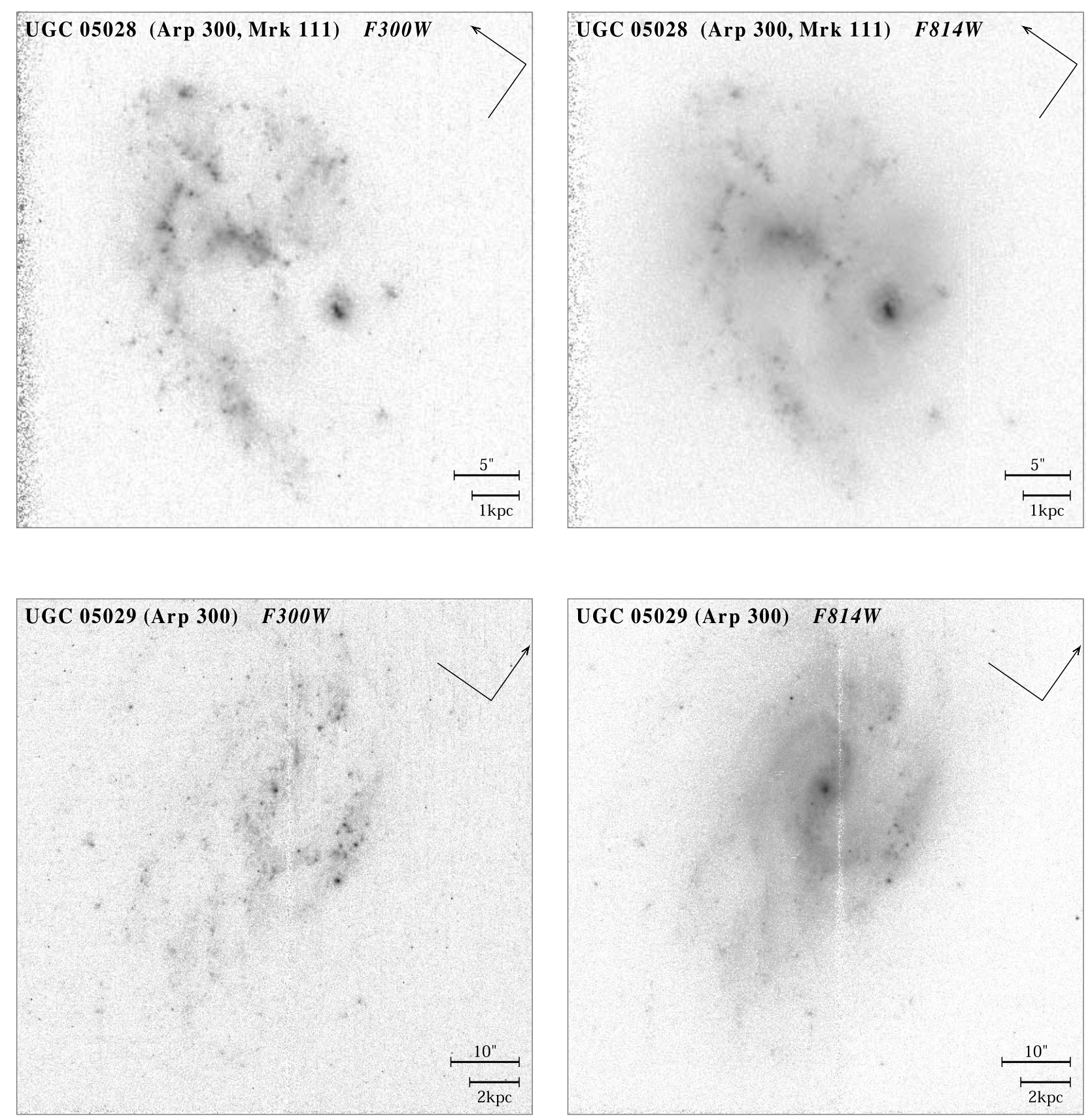

FIG. 3.-3.23a. UGC 05028 (top): WFPC2 F300W and F814W. 3.23b. UGC 05029 (bottom): WFPC2 F300W and F814W.

3.25. ESO 418-G008 $(\mathrm{T}=8$; $\mathrm{Sdm})$.- - This is a small latetype spiral galaxy that is resolved in at least its brighter starforming regions (Fig. 3.25), which are mostly distributed along its outer perimeter and perhaps constitute the beginning of spiral structure. The F814W image also suggests a faint bar running along the minor axis that may be connected to this spiral structure. The $B$ and $V$ images of Matthews \& Gallagher (1997) show that ESO 418-G008 has a bright, prominent bar (see also Fig. 4.25). A faint low-SB structure is visible in F814W that is spread more smoothly throughout the galaxy, presumably its unresolved older stellar population. A color map made from our HST data ${ }^{8}$

\footnotetext{
${ }^{8}$ See http://oposite.stsci.edu/pubinfo/pr/2001/04.
}

reveals a very faint red nucleus. Such compact nuclei are common features of extreme late-type spirals (Matthews \& Gallagher 1997; Matthews et al. 1999; Böker et al. 2001). The nucleus of ESO 418-G008 was not previously visible in ground-based images (Matthews \& Gallagher 1997), but Böker et al. (2001) have recently imaged it in the F814W filter with the WFPC2 PC. They measure $M_{I}=-10.24 \mathrm{mag}$, assuming a distance of $14.1 \mathrm{Mpc}$.

The galaxy appears to have sharp "edges" in both filters, but this may be largely a visual impression caused by the single star-forming region to the lower left (north) in Figure 3.25. The ground-based $U B V R$ images show similarly an outer spiral-like structure that becomes less and an inner bar that becomes more pronounced at longer wavelengths. 

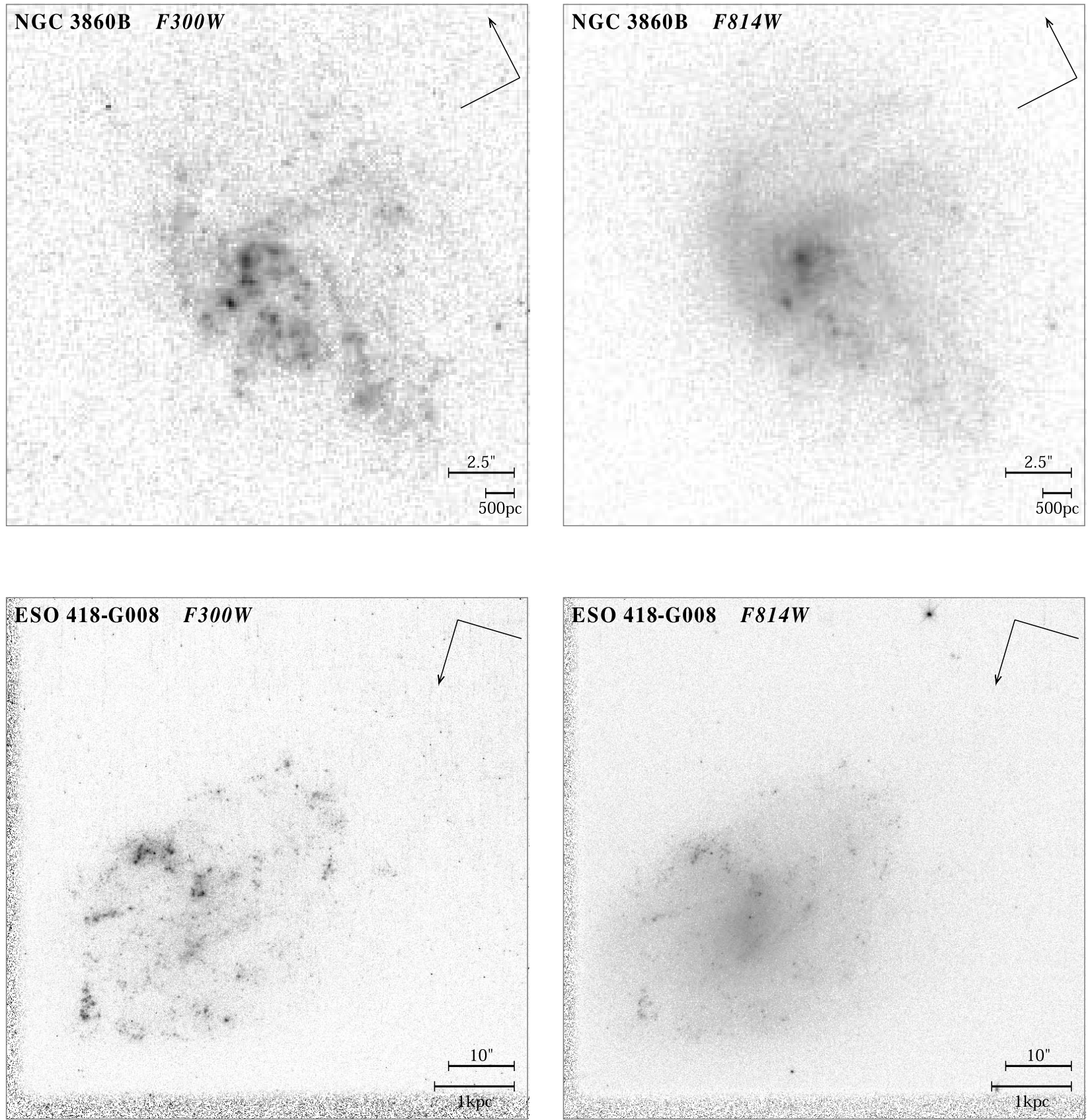

FIG. 3.-3.24. NGC 386B (top): F300W and F814W. 3.25. ESO 418-G008 (bottom): WFPC2 F300W and F814W.

It is a late-type galaxy with a modest morphological $K$-correction.

3.26. NGC $1510(\mathrm{~T}=-2.3$; $\mathrm{S} 0 \mathrm{pec})$.- This is a small blue, amorphous galaxy (Eichendorf \& Nieto 1984). NGC 1510 is a physical companion to the much larger SB galaxy NGC 1512, with which it is interacting (Hawarden et al. 1979). The early-type classification in the RC3 probably resulted from ground-based seeing hiding most of the irregular nature of this object. No clear bulge is visible. NGC 1510 has fairly similar appearance in F814W and F300W, but the F814W image shows more of a diffuse component, while the $\mathrm{F} 300 \mathrm{~W}$ resembles more a disklike component seen edge-on and shows some linear dust features. The object is resolved in at least its brighter star-forming regions. Scans of ground-based photographic plates in $B$ (from Lauberts \& Valentijn 1989, as obtained through NED) and in $R_{F}$ (from the second-generation Digital Sky Survey) are substituted for CCD images in Figure 4.26.

\subsection{Magellanic Irregulars}

3.27. NGC 5253 (a.k.a. Haro 10; $\mathrm{T}=10$; Im).- $\mathrm{NGC}$ 5253 is a peculiar I0 galaxy or type II irregular. This object has a very similar appearance in F300W and F814W and is clearly resolved into individual bright star-forming regions or stars. Table 1 shows that it is one of the closest galaxies in 

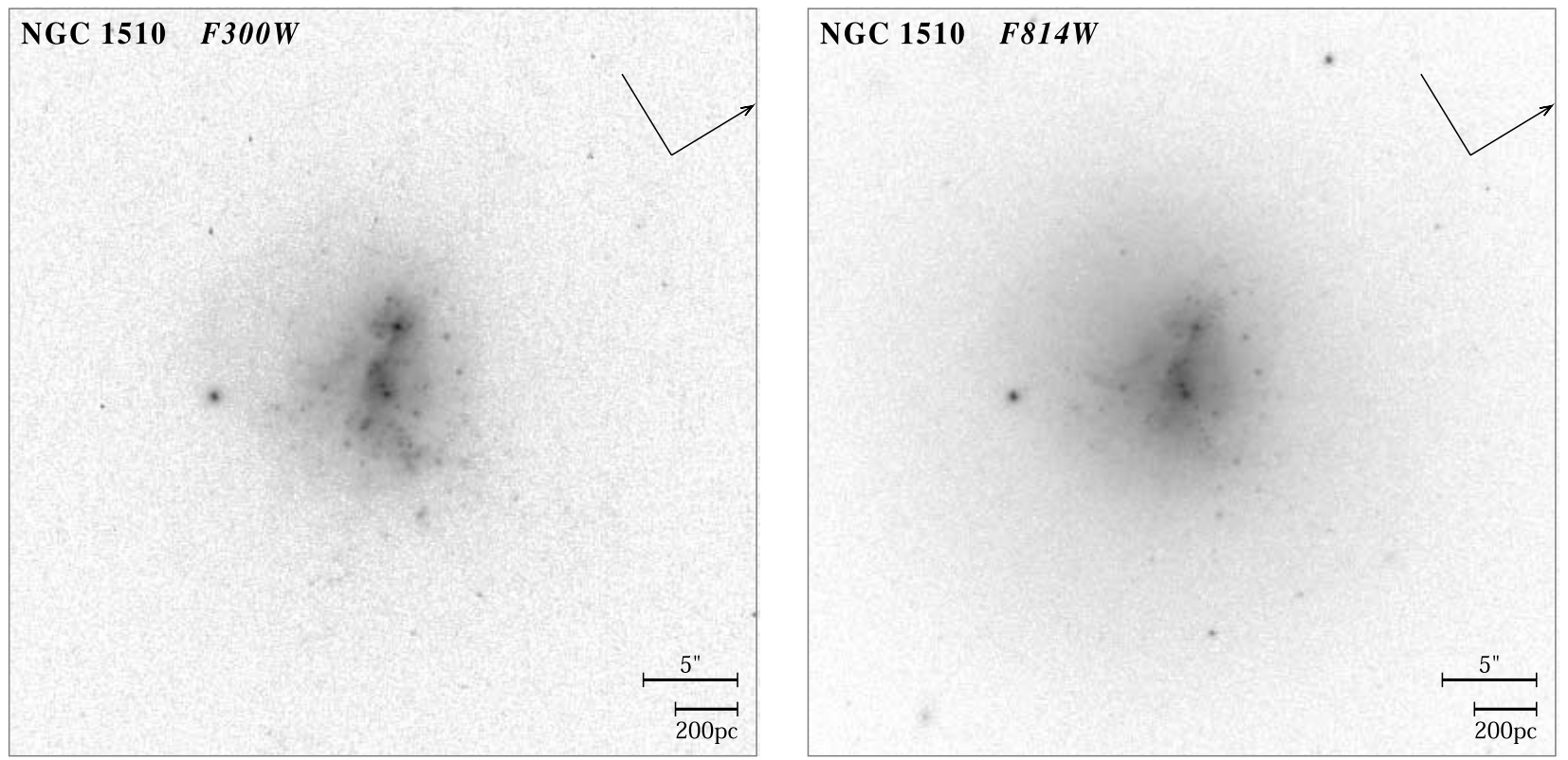

FIG. 3.-3.26. NGC 1510: WFPC2 F300W (left) and F814W (right).

our sample. The dust lane that appears to emerge from one side of the center outward is visible at all wavelengths, including in the lower resolution ground-based $U B V R$ images (Fig. 4.27). Overall, this object has a largely similar appearance from UIT $150 \mathrm{~nm}$ through the ground-based $K$ band, with some mild wavelength dependence modulations due to dust patches.

This object was first described after discovery of a nearby nova (Hubble \& Lundmark 1922; Gaposchkin 1936). NGC 5253 is a well-studied nearby starbursting galaxy at a distance of 4.1 Mpc (Sandage et al. 1994). It is typically classified as an amorphous or irregular galaxy but has outer isophotes reminiscent of a dwarf elliptical (Sersic, Carranza, \& Pastoriza 1972; Caldwell \& Phillips 1989). It has a starburst located at its center, with dozens of intense blue starforming clusters (Caldwell \& Phillips 1989; Storchi-Bergmann, Kinney, \& Challis 1995; Calzetti et al. 1995). NGC 5253 also contains an unusual $\mathrm{H} \alpha$ morphology (Marlowe et al. 1995; Calzetti et al. 1995) with loops and filaments and a diffuse component that is at least partially produced from shocks (Calzetti et al. 1999).

Recent HST images in optical broadband and line emission show that this object is a small starbursting galaxy (Beck et al. 1996; Calzetti et al. 1997, 1999; Gorjian 1996). The galaxy was detected by IRAS (IRAS 13370-3123 in the IRAS Point Source Catalog; Beichman et al. 1988) and is a weak radio source in the Parkes-MIT-NRAO $4.85 \mathrm{GHz}$ Survey (Wright et al. 1996) and also had a FAUST far-UV point source 0.5 from the galaxy (Bowyer et al. 1995). Other recent studies include a systematic H I study (Kobulnicky \& Skillman 1995), radio observations of a starbursting knot that has no optical counterpart (Turner, Ho, \& Beck 1998; Turner, Beck, \& Ho 2000), as well as a CO map (Turner, Beck, \& Hurt 1997), ROSAT X-ray studies of multiple superbubbles in its starburst nucleus (Strickland \& Stevens 1999), and Infrared Space Observatory observations of its hot stars and Wolf-Rayet-like outflow (Crowther et al. 1999), among others.
Only $130 \mathrm{kpc}$ away is the larger spiral galaxy NGC 5236 (M83), which also experiences a starburst in its nuclear regions. NGC 5253 has a large outer distribution of $\mathrm{H}$ i gas that could be feeding the central starburst in M83 (Kobulnicky \& Skillman 1995). In addition, the bar in M83 might be triggering its central starburst. However, there is evidence that the starbursting properties in this system are produced in part by a dynamical interaction between the two objects (Caldwell \& Phillips 1989).

3.28. NGC 1140 (a.k.a. Mrk 1063, VV 482; $\mathrm{T}=10$; Im).-This is an irregular galaxy. This object has a fairly similar appearance from the mid-UV through the $I$ band (Fig. 4.28). In F300W and F814W (Fig. 3.28) the object is resolved in at least its brighter star-forming regions. The "super-star clusters" visible here were studied using $H S T$ optical-band imaging by Hunter, O'Connell, \& Gallagher (1994). The larger FOV in the ground-based images also shows a trail of knots to the southwest, possibly an infalling dwarf companion.

3.29. UGC $05626(\mathrm{~T}=10 ; \mathrm{Im})$. - This is an irregular galaxy. The object is resolved in at least its brighter star-forming regions, which are mostly draped around its edges as seen in F300W. The bluest and brightest star-forming knots to the left (north) are also visible in F255W. In F814W, the object also shows a faint low-SB structure that is spread more smoothly throughout the galaxy, presumably its unresolved older stellar population. No central bulge is visible at any wavelength. The structure in the ground-based $U B V R$ images (Fig. 4.29) is similarly independent of wavelength and shows the outer rings of knots, resembling the beginning of spiral structure. It is curious that such spiral structure would start before even a small central bulge or bar has formed and leads one to wonder about the dark matter content of (the central parts of) this galaxy.

3.30. UGC $09855(\mathrm{~T}=10$; $\mathrm{Im})$.- This is a Magellanictype irregular. The object is resolved in at least its brighter star-forming regions. The brightest of these regions are also visible in F255W. The F814W shows a faint central bulge 

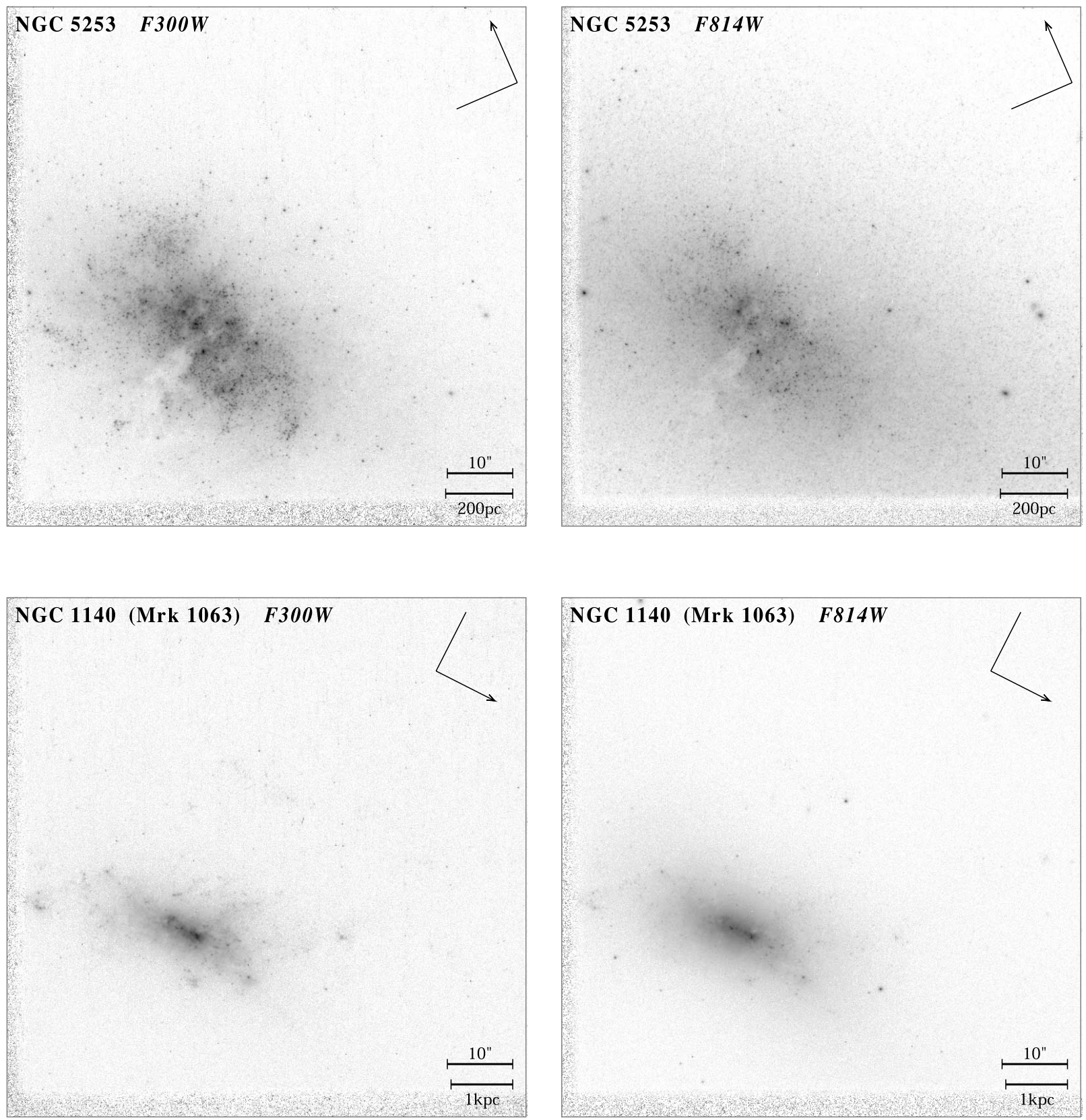

FIG. 3.-3.27. NGC 5253 (top): WFPC2 F300W and F814W. 3.28. NGC 1140 (bottom): WFPC2 F300W and F814W.
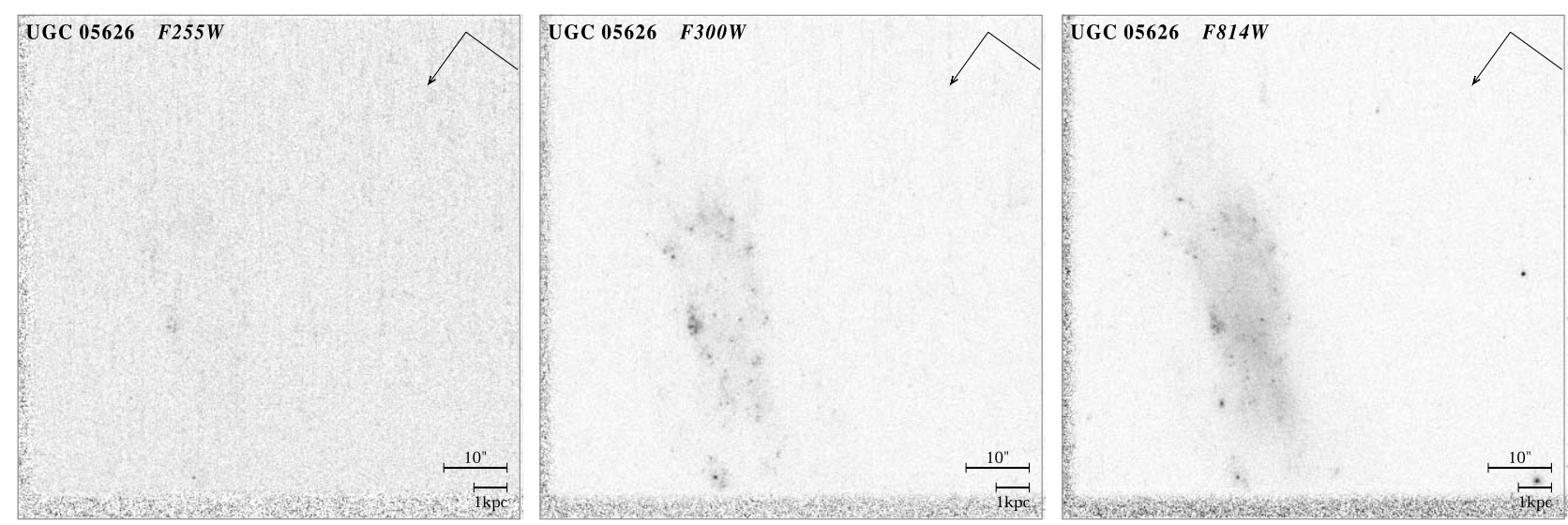

FIG. 3. - 3.29. UGC 05626: WFPC2 F255W (left), F300W (middle), and F814W (right). 

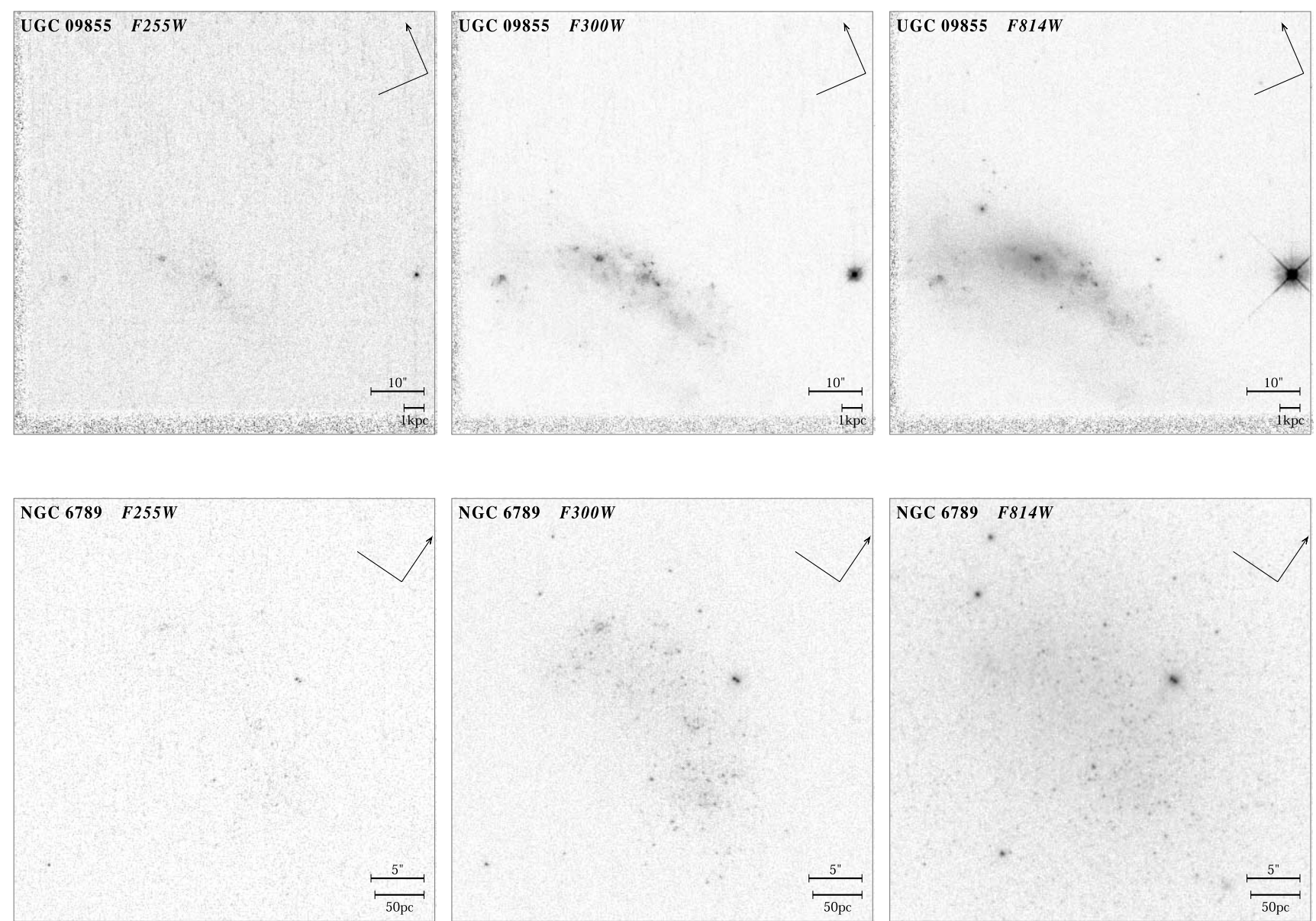

FIG. 3.-3.30. UGC 09855 (top): WFPC2 F255W, F300W, and F814W. 3.31. NGC 6789 (bottom): WFPC2 F255W, F300W, and F814W.

that is not quite aligned with the geometrical center of the object (see also the ground-based images in Figure 4.30 and the disk of UGC 05626 in the previous paragraph).

3.31. NGC $6789(\mathrm{~T}=10 ; \mathrm{Im})$.- This is a Magellanic-type irregular. The object is resolved in at least its brighter starforming regions. The brightest ones of these are also (but barely so) visible in F255W. The structure in the groundbased $U B V R$ images (Fig. 4.31) is rather independent of wavelength, and no central bulge is visible at any wavelength.

3.32. Mrk $66(\mathrm{~T}=11 ; \mathrm{Pec})$.- This object shows a significant number of blue star-forming knots; the one to the left (west) of the center (Fig. 3.31, F814W) may be part of a faint small bulge. The knots to the right (east) are isolated from the main object in both filters and may be separated from the main body by a dust lane or pocket, as is also seen in the ground-based images (Fig. 4.32). It is not clear whether this galaxy is a small linear object or a late-type galaxy seen edge-on.

The ground-based images provide some additional clues, since in all $U B V R$ filters, a low-SB disk or halo is seen surrounding the rather linear structure seen in F300W. If this rounder feature were a halo, then Mrk 66 could be a triaxial amorphous galaxy, although the fact that this feature is also visible in the ground-based $U$-band filter below the $4000 \AA$ break would imply a rather young halo age of $\lesssim 1-2$ Gyr.
Alternatively, if this rounder feature is a face-on disk, then the linear feature that is primarily visible in $\mathrm{F} 300 \mathrm{~W}$ could be a star-forming bar. In that case, the rounder feature would have to be a rather old disk ( $\sim 1$ Gyr), since no spiral arm structure is visible at the same location in the F300W image. Spatially resolved spectroscopy and kinematics will be necessary to distinguish between these possibilities.

In combination with the HST F300W and F814W images, the ground-based $U B V R$ images confirm that, to first order, this object has little morphological $K$-correction; i.e., its appearance is rather similar at most wavelengths. However, significant SB dimming at high redshifts could change a roundish object like Mrk 66 as seen in visible light into a more linear object in the mid-UV and so help explain the appearance of some of the "chain "galaxies seen at high redshifts (Cowie, Hu, \& Songaila 1995; see also Dalcanton \& Shectman 1996).

3.33. UGC 05189 (a.k.a. VV 547; T =10; Im).-Like Mrk 66, the HST images of this object show a significant number of blue star-forming knots. The ground-based $U B V R$ images (Fig. 4.33) show that the arc imaged with WFPC2 is in fact part of a much larger, low-SB structure. This is clearly a very dynamically disturbed system. There is no obvious central bulge. In combination with the HST F300W and F814W images, the ground-based UBVR images confirm that this object has almost no morphologi- 

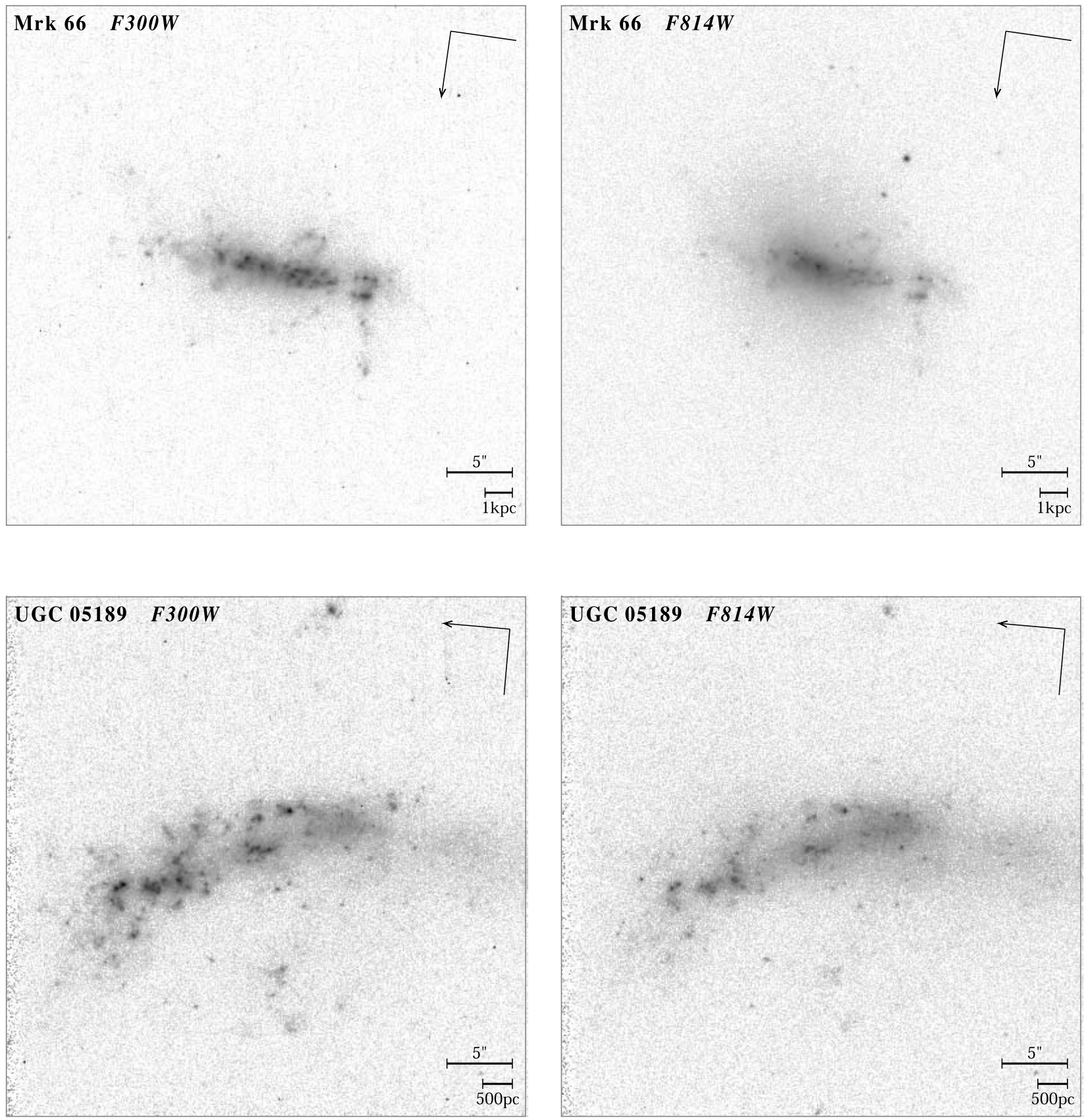

FIG. 3.-3.32. Mrk 66 (top): WFPC2 F300W and F814W. 3.33. UGC 05189 (bottom): WFPC2 F300W and F814W.

cal $K$-correction; i.e., its appearance is about the same at all wavelengths. Its light must be dominated by young hot stars throughout.

\subsection{Interacting, Peculiar, and Merging Galaxies}

3.34. NGC 3690/IC 0694 (a.k.a. Arp 299, VV 118; T = 9; $\mathrm{Sm})$.- This is a merger of two late-type disk galaxies. The object has a very similar morphology in F814W, F300W, and $\mathrm{F} 255 \mathrm{~W}$, although the sensitivity in $\mathrm{F} 255 \mathrm{~W}$ is much reduced. Comparison of the F300W and F814W images reveals significant dust patches, most of which are irregularly distributed, as shown in the enlargements in Figure 3.34b. This figure also shows the numerous young superstar clusters spread throughout the system (e.g., Meurer et al. 1995).

The objects somewhat resemble the Antennae galaxies (NGC 4038/39), but the encounter geometry is more complicated and the merger is slightly more advanced in Arp 299. H I and CO kinematics suggest a prograde-retrograde or prograde-polar encounter (Hibbard \& Yun 1999a; Casoli et al. 1999). There are no obvious bulges in the mid-UVoptical images, but near-infrared imaging reveals a bulge in at least the easternmost system (Smith et al. 1996a), which is quite hidden by dust in all filters blueward of $\sim 1 \mu \mathrm{m}$. Several of the bluest star-forming knots shine through clearly in 

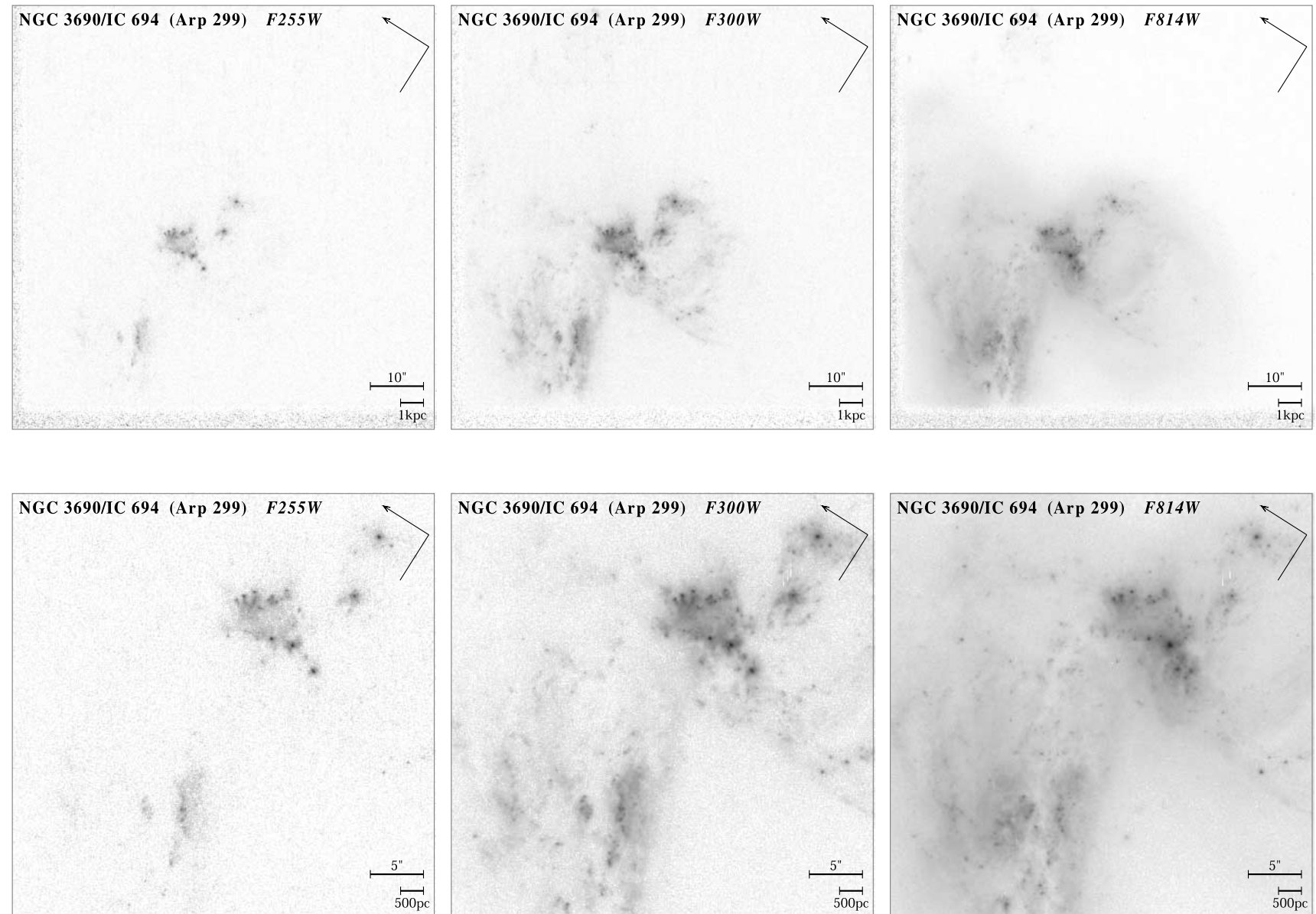

FIG. 3. - 3.34a. NGC 3690/IC 694 (top): WFPC2 F255W, F300W, and F814W. 3.34b. NGC 3690/IC 694 (bottom): enlargement of the central portion of this merging galaxy pair, emphasizing the dusty patches and star-forming knots.

F300W and F255W, although most of the galaxy shows significantly dusty regions, where such starbursting knots would be much more reddened.

3.35. UGC 06527 (a.k.a. Arp 322, Mrk 176, VV 150, HCG 056; $\mathrm{T}=11$; Pec).- This is a spiral galaxy in a Hickson compact group that is undergoing a merger with at least one of its companions (e.g., Fasano \& Bettoni 1994; Allam et al. 1996). Its spiral disk and arms are distorted into tidal tails because of this encounter, as also implied by numerical simulations of such events (e.g., Barnes \& Hernquist 1992). When comparing F814W to F300W, the inner bulge shows a dusty inner disk-like structure. The galaxy is not resolved into its brightest star-forming regions or stars, as a result of its larger distance. The ground-based images in Figure 4.35 suggest a small morphological $K$-correction, if any.

3.36. UGC 08335 (a.k.a. Arp 238, VV 250; T = 4; Sbc).This system consists of two strongly interacting disk galaxies of approximately equal luminosity. Both disks are distorted during this encounter and display tidal tails (Fig. 4.36), suggesting a prograde-prograde encounter (e.g., Barnes \& Hernquist 1992). The RC3 morphological type probably results from confusing the tidal arms with spiral structure or the two nuclei with a central bar (Fig. 4.36) in ground-based images. When comparing F814W to F300W, the inner bulges of both systems show significant dust lanes, possibly from gas-rich material raised during the encounter. The system is too distant to be resolved into its brightest star-forming regions or stars.

3.37. IC 2184 (a.k.a. Mrk 8, VV 644; T =9; Sm).-The "flying V" is probably a merger of two disk galaxies seen nearly edge-on, as indicated by the presence of pronounced tidal tails in deep ground-based images (Gallagher et al. 2000). Star formation is active in most of both of the disks, and in the F255W image only the brightest star-forming knots are seen as a result of the reduced sensitivity of WFPC2 in this filter. Dust patches are visible in F814W, corresponding to reduced or no light in F300W and F255W at those locations. The ground-based $U B V R$ images (Fig. 4.37) similarly show a very modest dependence of the morphology on rest-frame wavelength.

3.38. NGC 5278/79 (a.k.a. Arp 239, Mrk 271, VV 19; $\mathrm{T}=3 ; \mathrm{Sb})$. - This is one of the most curious objects in our sample. Like UGC 08335, this system consists of two strongly interacting spiral galaxies. The encounter clearly distorts both galaxy disks, resulting in tidal tails and exciting a strong $m=1$ mode in the larger of the two galaxies, similar to features seen in numerical simulations of such events (Zeltwanger, Comins, \& Lovelace 2000; see also Phookun et al. 1992). When comparing F814W to F300W, both the inner bulges show significant dust lanes. Part of the 

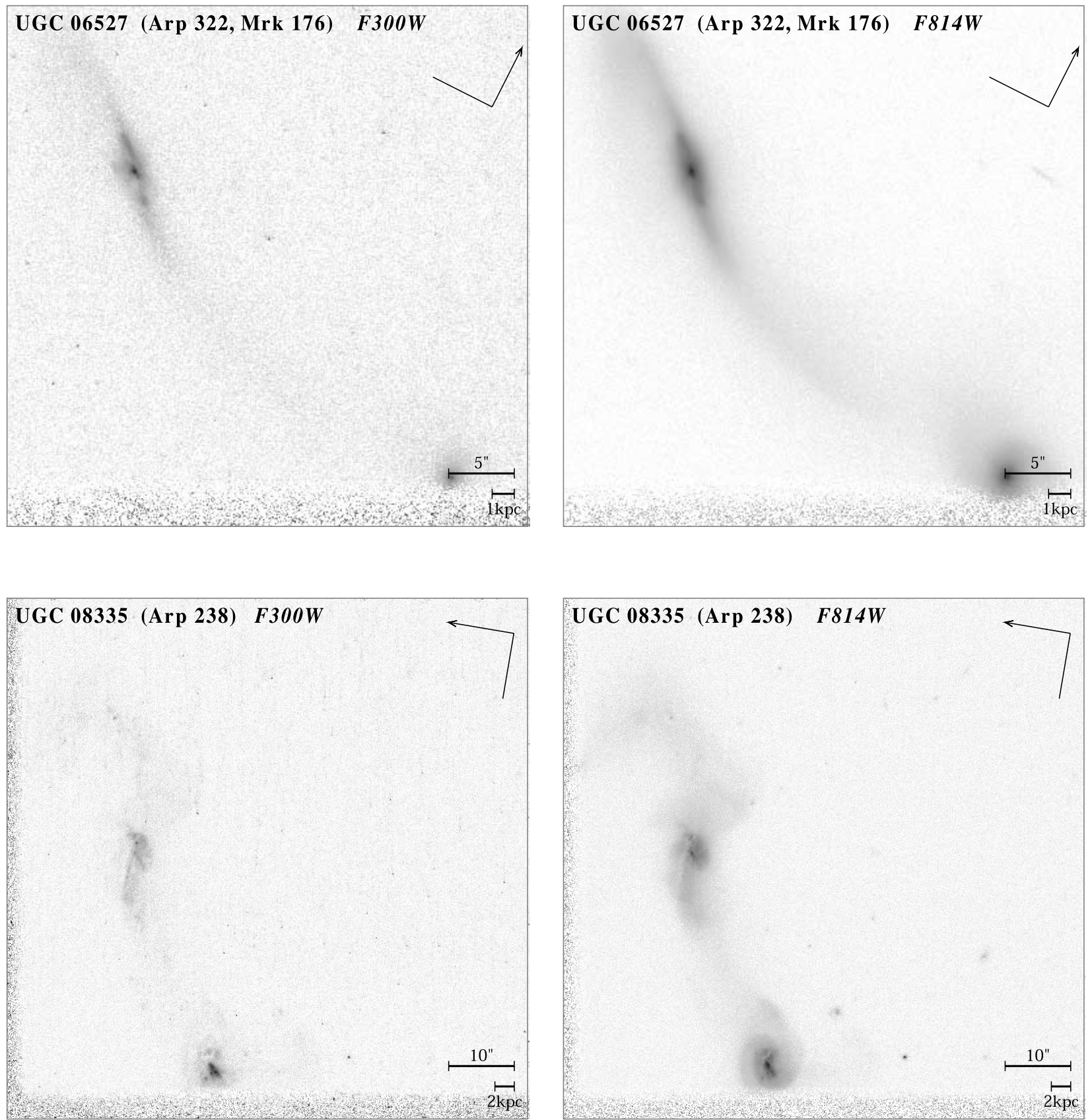

FIG. 3.-3.35. UGC 06527 (top): WFPC2 F300W and F814W. 3.36. UGC 08335 (bottom): WFPC2 F300W and F814W.

dust appears to be pulled out along with one of the spiral arms in the tidal encounter, possibly as a result of a shock related to this encounter.

Most remarkable is the very curved thin dust lane, best seen in $R$ and $\mathrm{F} 814 \mathrm{~W}$, that drapes across the bigger galaxy. This dust lane starts somewhere in the southern spiral arm of the bigger galaxy (upper or southern part in the WFPC2 CCD as shown in Fig. 3.38), curves all the way across the small nuclear bulge of this galaxy, and makes it into the spiral arm to the lower left that appears to connect to the smaller galaxy. Careful comparison of the F300W and F814W images shows that the dust lane starts in what appears to be at least two funnel-like regions from the southern arm of the bigger galaxy. The dust in the southern arm appears to be distributed nonuniformly along the arm in several (three to four) sizeable "dust pockets or patches," which appear to merge into the dust lane and tidal tail that wraps around the bigger galaxy's nuclear bulge. A somewhat similar but much thinner dust trail was seen in $H S T$ images of the colliding nearby galaxy pair NGC 1409/1420 (Keel 2000), with material being pulled out between the two galaxies.

These dust pockets or patches are clearly visible in the data of the F300W images, and at the same location, the F255W images are entirely devoid of flux, lending credence to the conjecture that dust pockets cause these depressions 

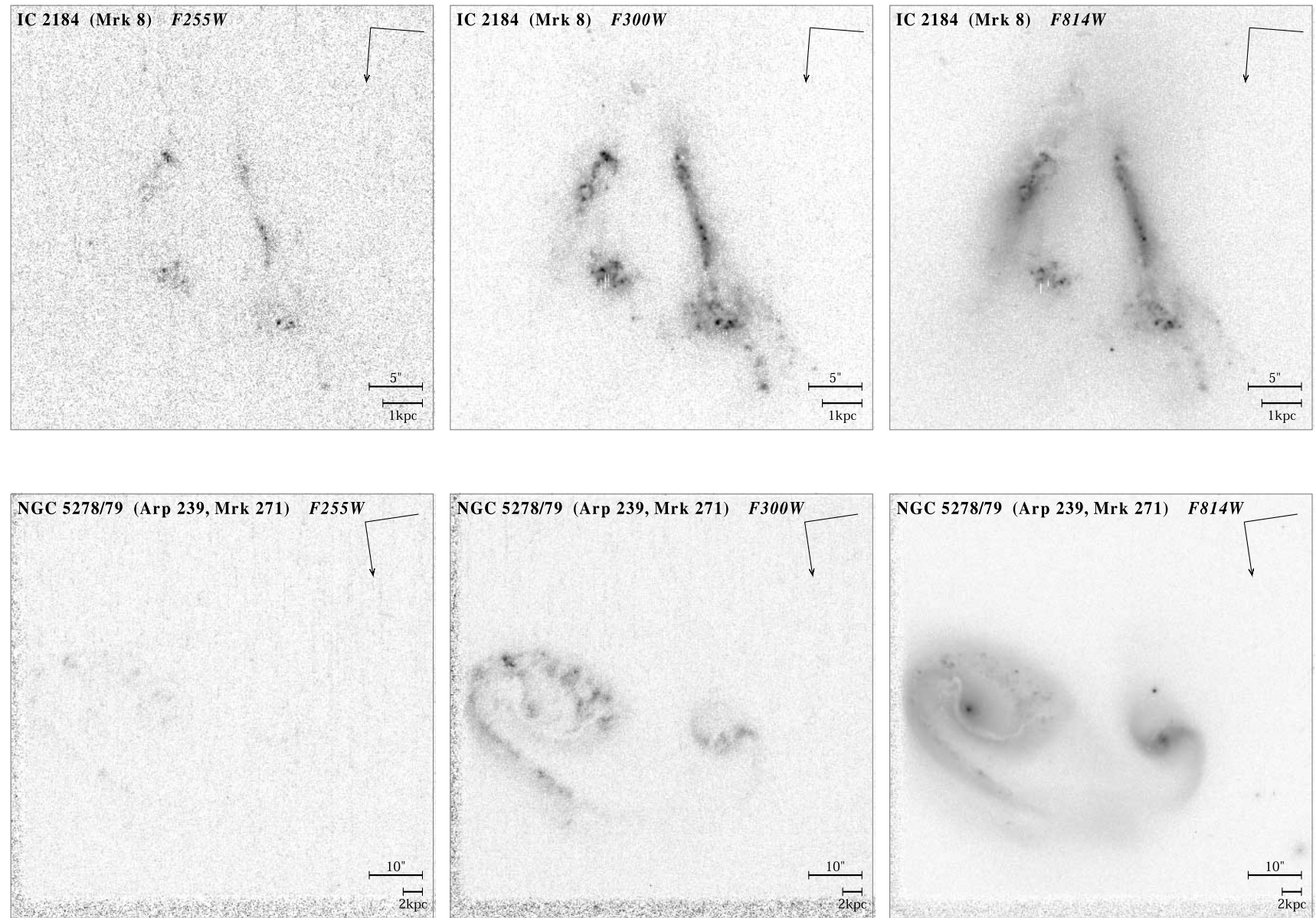

FIG. 3.-3.37. IC 2184 (top): WFPC2 F255W, F300W, and F814W. 3.38. NGC 5278/79 (bottom): WFPC2 F255W, F300W, and F814W.

in the light distribution. The patches are best seen by blinking the F255W, F300W, and F814W images in rapid succession, each displayed at an appropriate logarithmic stretch. The bluer ground-based images (Fig. 4.38) show a similarly diminished surface brightness at the location of the dust patches.

\section{DISCUSSION OF GENERAL TRENDS IN THE WFPC2 MID-UV IMAGES}

Here we discuss the subsamples of each of the Hubble types separately. The light profiles of all types will be presented in a subsequent paper in conjunction with the ground-based $U B V R$ profiles, after all the ground-based zero points have been secured (see Table 2 ).

\subsection{Early-Type Galaxies}

The regular early-type galaxies (elliptical and $\mathrm{S} 0$ galaxies) in our sample show in general a significant change in SB from the mid-UV to the red, reflecting an overall lack of a young stellar population (CGCG 097-094, NGC 1396, NGC 4478; $\S 3.1$ ). Typically, the $\mu_{\mathrm{F} 300 \mathrm{~W}}$ of $\mathrm{E} / \mathrm{S} 0$ galaxies is 3-5 mag arcsec ${ }^{-2}$ fainter than $\mu_{\mathrm{F} 814 \mathrm{~W}}$. As was also seen in the far-UV UIT sample of Marcum et al. (2001) and Kuchinski et al. (2000), they are dominated by an older stellar population and so are generally faint in F300W. Out of seven early-type galaxies imaged, two have small blue nuclear features (UGC 03426, NGC 3921; $\S \S 3.2$ and 3.3). UGC 03426 is a Seyfert 2, and so this feature could be associated with a bipolar outflow. The other, NGC 3921, is a well-studied merger remnant from the Toomre sequence, and the blue nuclear feature could indicate a location of residual star formation that is only partially obscured by dust. Three early-type galaxies (NGC 1396, NGC 3516, and UGC 08823; $\S \S 3.1$ and 3.2) become dominated by point sources in the mid-UV, indicating weak optical-UV AGNs, Seyfert nuclei, or LINER nuclei. While these are small number statistics, their presence in our current small sample of early-type galaxies is due to our selection of galaxies with high predicted average SB in the mid-UV within their effective (i.e., half-light) area ( $\$ 2.2 .3)$. This resulted in including a number of objects in our sample that are dominated in the $U$ band and mid-UV by AGNs.

If AGNs generally reside in bulge-dominated galaxies (e.g., Magorrian et al. 1998), then the red and old stellar population of the underlying early-type galaxies will be generally faint in the UV, but the presence of a (weak) AGN will result in blue $(U-B)$ colors and therefore a high SB in the mid- to near-UV, and so inclusion into UV-selected samples. Similarly, ground-based $U$-band-selected surveys would result in significant numbers of AGNs in unrecognized early-type galaxies at moderate redshifts $(z \simeq 0.3)$. At redshifts of a few tenths, the ground-based $U$-band selection would similarly show these objects essentially as point sources, just like some of the nearby early-type galaxies selected 

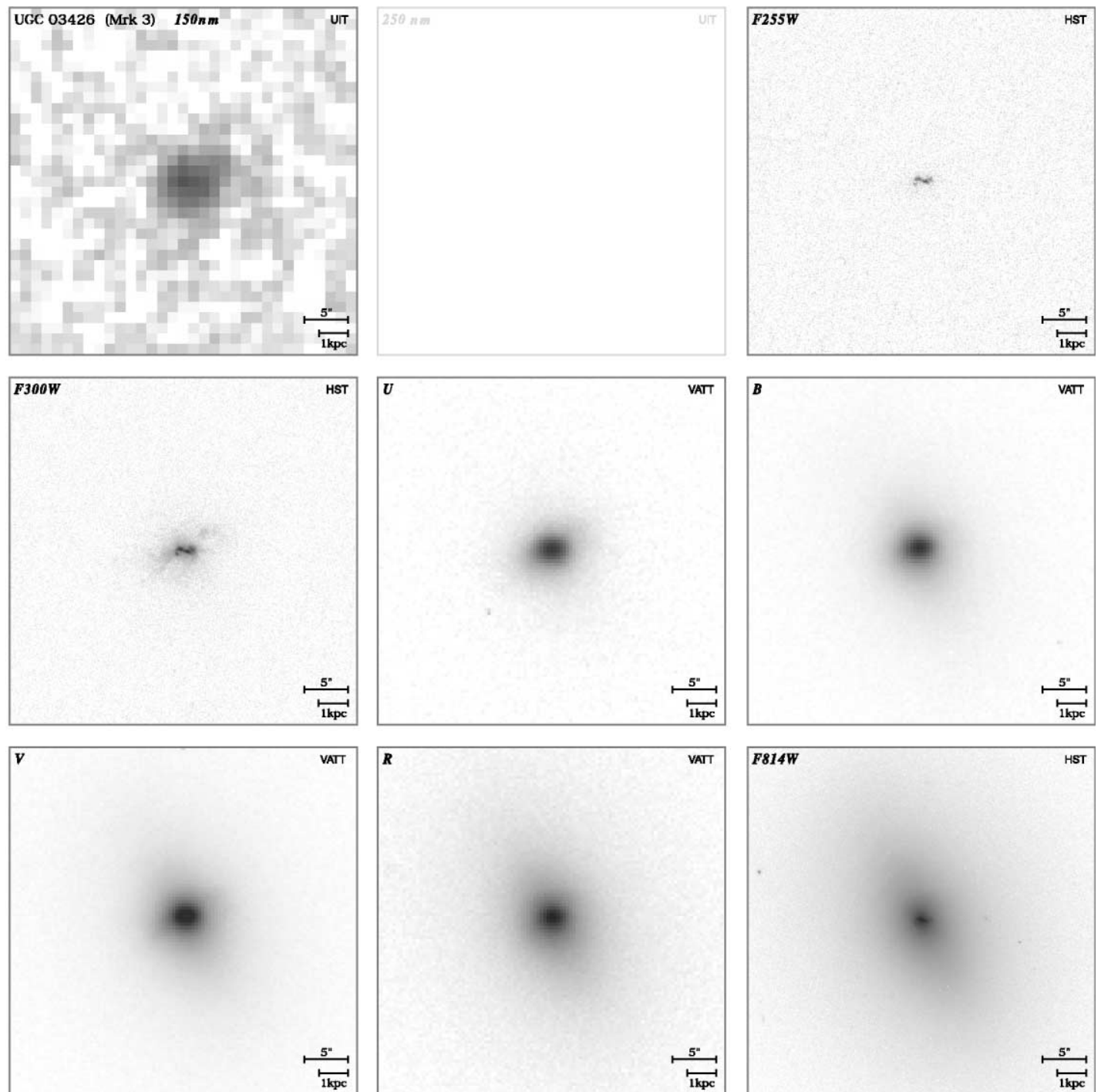

FIG. 4.-Panchromatic atlas of far- and mid-UV (UIT), mid-UV (HST/WFPC2), and ground-based optical UBVR and near-IR $J H K$ (ground or $H S T /$ NICMOS) observations of the 37 nearby galaxies in the present sample. As in Fig. 3, the galaxies are sorted and numbered according to morphology, and each of the gray-scale renditions is displayed using a logarithmic stretch. Unlike Fig. 3, all images have been rotated to have north up and east to the left and are presented on a common plate scale. [The printed version contains only a few example pages; see the electronic edition of the Supplement for all additional figures.] 4.05. UGC 03426: UIT $150 \mathrm{~nm}$ and HST/WFPC2 F255W (top), HST/WFPC2 F300W and ground-based $U$ and $B$ (middle), and ground-based $V$ and $R$ and HST/WFPC2 F814W (bottom).

in our HST mid-UV sample. However, had these objects been selected at $z \simeq 0.3$ from the ground at much longer wavelengths (i.e., in the $I$ band), then they would still have shown up as early-type galaxies in images with good ground-based seeing. This leads us to wonder to what extent the (strong) cosmological evolution of AGNs selected optically or through their near-UV excess at modest redshifts (Koo \& Kron 1988; Boyle \& Terlevich 1998; Boyle et al. $2000)$ could in part be due to a morphological $K$-correction of early-type galaxies with weak AGNs. A further discussion of the galaxy light underlying low-redshift QSOs is given by Bahcall, Kirhakos, \& Schneider $(1995,1996)$, Bahcall et al. (1997), Kirhakos et al. (1999), and Maoz et al. (1996), who determine the AGN components seen with WFPC2 in early- to mid-type galaxies.

Two more galaxies, UGC 05101 and UGC 08696 (§ 3.3), are tentatively placed in this section of early-type galaxies.
These objects are merger remnants and have significant dusty disks in the mid-UV at present, but we believe they will soon evolve into early-type galaxies.

\subsection{Mid-Type Spirals}

In general, spiral arms are more pronounced in the midUV, as UIT has shown to be generally true in the far-UV (Bohlin et al. 1991; Hill et al. 1992; Kuchinski et al. 2001; Marcum et al. 2001), but mid-type spirals and star-forming galaxies (e.g., NGC 6753 and NGC 3310; § 3.5) appear more similar from the mid-UV to the optical than the early-type galaxies discussed above. However, several appear as later types in the mid-UV (e.g., NGC 7769; $\S 3.5$ ), and a few show drastic changes in type from the optical to the mid-UV, equivalent to an apparent change in morphological type $\Delta \mathrm{T} \gtrsim 3$ (e.g., NGC 2551 and NGC 7685; $\S \S 3.4$ and 3.5). 

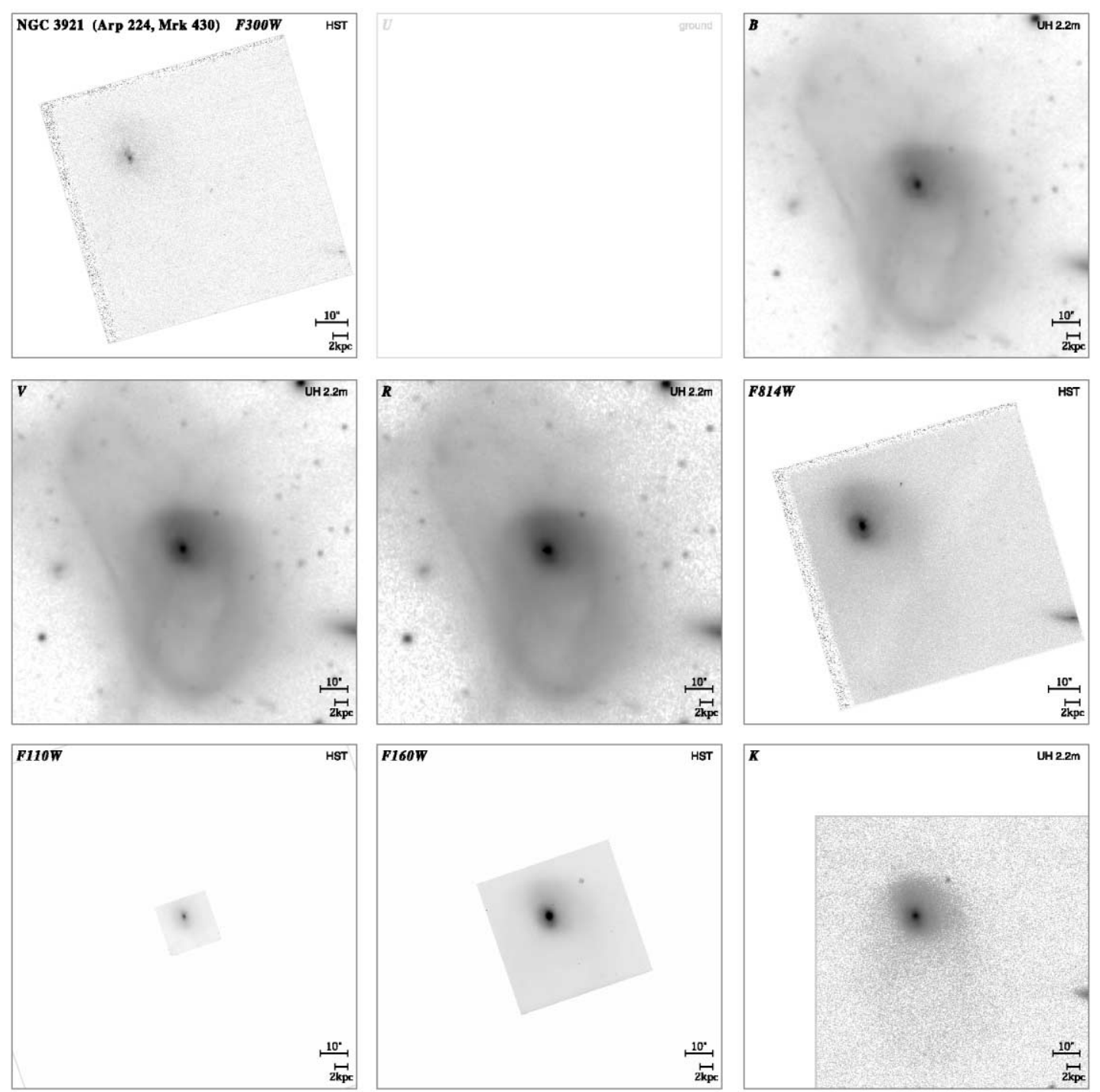

FIG. 4. - 4.07. NGC 3921: HST/WFPC2 F300W and ground-based $B$ (top), ground-based $V$ and $R$ and $H S T /$ WFPC2 F814W (middle), and $H S T /$ NICMOS F110W $(J)$ and F160W $(H)$ and ground-based $K($ bottom).

One galaxy shows a spectacular resonance ring full of hot stars (NGC 6782; §3.6), while the remainder of the disk becomes essentially invisible in the mid-UV. This object is discussed in detail by P. B. Eskridge et al. (2002, in preparation).

We see a variation in color of the galaxy bulges/centers in the spirals and a considerable range in scale and SB of the individual star-forming regions. Dust features in mid-type spirals can be well traced by comparing the F300W to the F814W images. Dust is visible in lanes or patches (possibly trailing spiral density wave patterns?), pockets, and/or bubbles. A curious feature is that almost without exception, the mid-type spirals in our sample have their small nuclear bulges bisected by a dust lane, which is often connected to the inner spiral arm structure. This is interesting in the context of the recent finding that all bulge-dominated systems have a central black hole with an average black hole mass $M_{\text {bh }} \simeq 0.005 M_{\text {bulge }}$ (Magorrian et al. 1998). The small nuclear dust lane seen by comparing the F814W and midUV images of almost all mid- to late-type spiral galaxies in our sample may then be involved in feeding the inner accretion disk (which is not visible in our HST images).

Some edge-on galaxies are very faint in F300W when compared to F814W (UGC 10043 and ESO 033-G22), others emit/transmit significantly more in F300W (UGC 06697 and IC 3949), while IC 4393 is an intermediate case (§ 3.7). All show an F300W/F814W ratio increasing from the inside out, as expected for a decreasing dust content from the inside out, and/or a strong radial gradient in the stellar population, if we assume that the dust and light sources are well mixed (i.e., the light sources are not preferentially located in front of most of the dust; see, e.g., Jansen et al. 1994; Witt \& Gordon 1996; Kuchinski et al. 1998).

\subsection{Late-Type Galaxies and Irregulars}

The late-type and irregular galaxies imaged are a heterogeneous mixture. The majority of these galaxies show an F300W morphology that is similar to that in F814W. Important differences are seen, however, as a result of recog- 

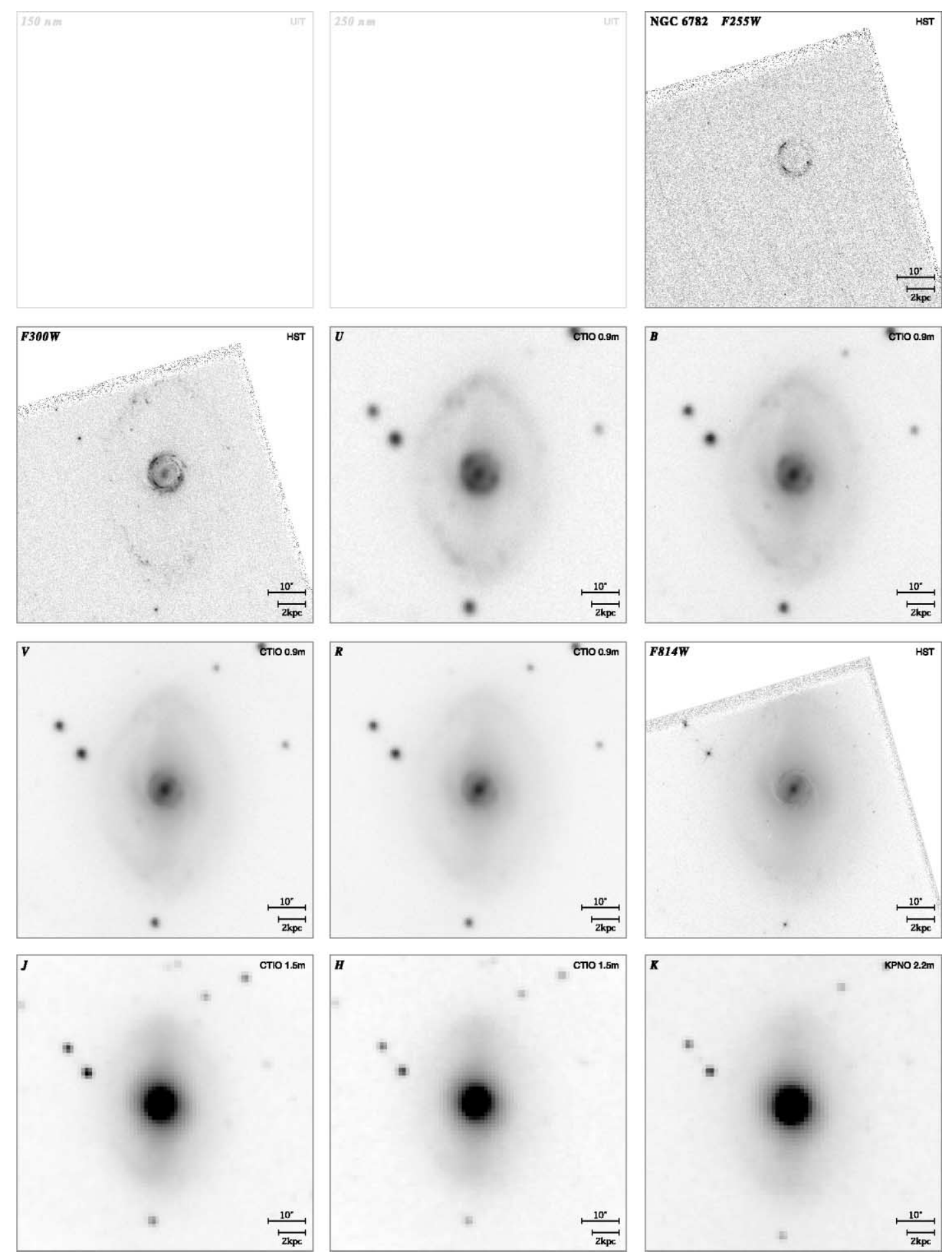

FIG. 4. 4.15. NGC 6782: HST/WFPC2 F255W (top), HST/WFPC2 F300W and ground-based $U$ and $B$ (second row), ground-based $V$ and $R$ and $H S T /$ WFPC2 F814W (third row), and ground-based $J, H$, and $K$ (bottom).

nizable dust lanes or pockets blocking out F300W light (e.g., UGC 06697, § 3.7; NGC 5253, UGC 09855, Mrk 66, § 3.9; Arp 299, IC 2184, § 3.10). Dust is visible in pockets, holes, or bubbles, perhaps as a result of supernova-induced outflows or outflows fueled by bright star-forming regions, such as seen in M82 at HST resolution (de Grijs, O'Connell, \& Gallagher 2001).

Some late-type galaxies are physically smaller galaxies with what appears to be the beginning of spiral structure (MCG +03-30-071, MCG +06-24-047, UGC 05028, NGC
3860B, ESO 418-G008, § 3.8; UGC 05626, § 3.9). Others are Magellanic irregulars (NGC 5253, NGC 1140, NGC 1510, UGC 09855, NGC 6789, Mrk 66, UGC 05189; $\S 3.8$ and 3.9) with various regions of stochastic star formation. Star formation ridges are commonly seen in the late-type galaxies, as well as hot stars or star clusters that are particularly conspicuous in the mid-UV (F300W and/or F255W).

A few late-type galaxies would be classified significantly differently when observed in the mid-UV than in the F814W passband (MCG +06-24-047, UGC 05626, UGC 09855; 

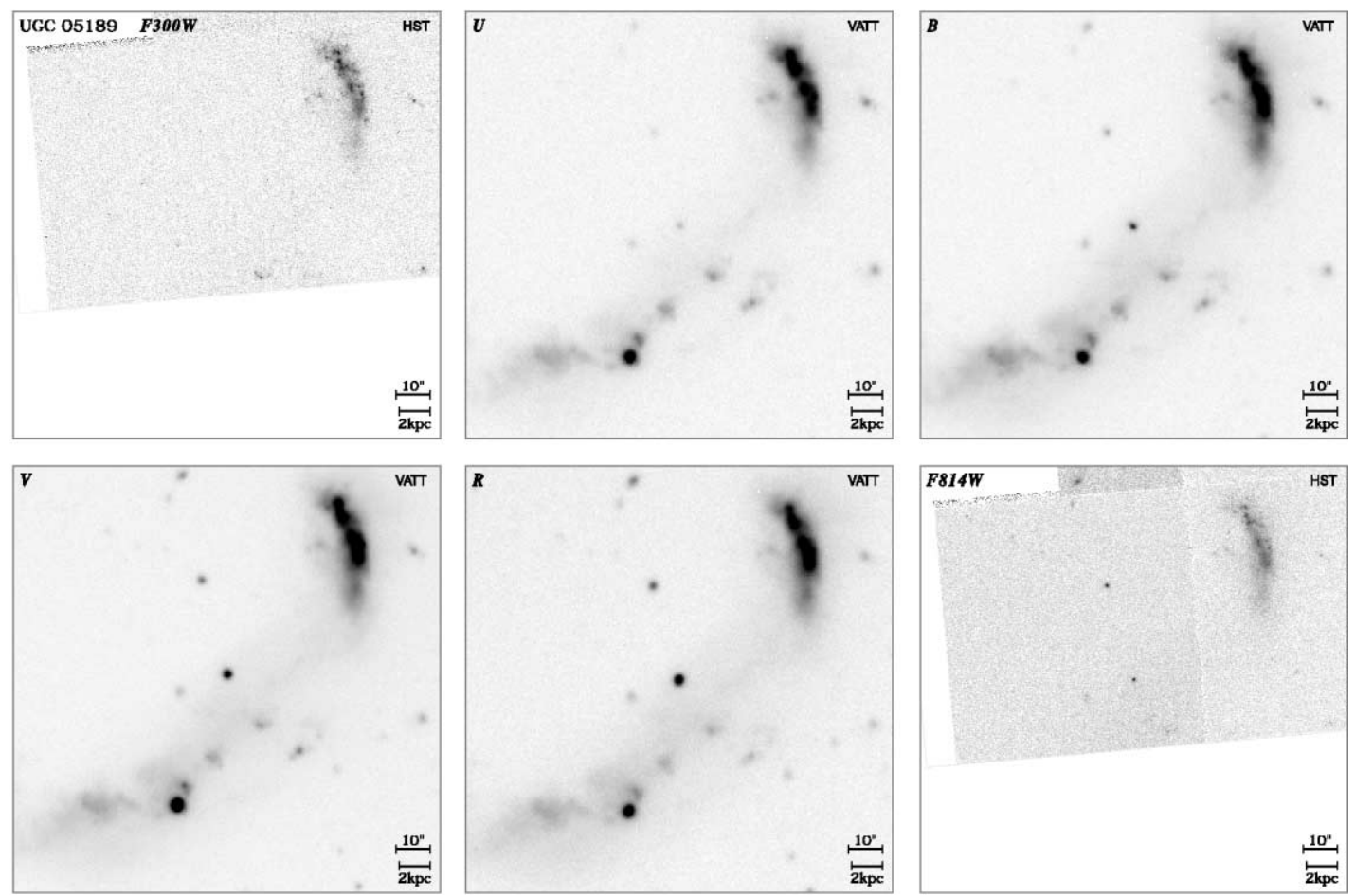

FIG. 4. - 4.33. UGC 05189: HST/WFPC2 F300W and ground-based $U$ and $B(t o p)$ and ground-based $V$ and $R$ and $H S T /$ WFPC2 F814W (bottom).

$\S \S 3.8$ and 3.9), especially when observed under less than perfect atmospheric seeing conditions. A quantitative discussion of the classification changes as a function of restframe wavelength will be given by S. C. Odewahn et al. (2003, in preparation).

\subsection{Peculiars and Mergers}

The majority of the peculiars and interacting galaxies (UGC 05189, Arp 299, UGC 06527, UGC 08335, IC 2184, Arp 239; $\S \S 3.9$ and 3.10) show an F300W morphology that is similar to that in F814W. Several mergers have spectacular dust lanes that thread along with the spiral arms, which are tidally distorted during the interaction (UGC 08335; Arp 239), while most others have absorption in apparently random locations associated with a starburst.

The HST images are in general not sufficiently deep nor do they sample a sufficiently large area on the sky to capture the lower SB features (e.g., low-SB tails and other large tidal features, faint extended disks or halos) that are well imaged on the ground-based photographic plates that were originally used to classify these objects. The inner regions of these objects reveal a wealth of structure in the HST images but often are almost entirely burned out or blurred on these ground-based images. The HST images thus do not necessarily reflect the distribution of the extended (evolved) stellar population. This is particularly true of the interacting systems, where tidal forces first and most easily affect the outer regions of the participants. Because interacting galaxies have both a disturbed distribution of old stars in their outskirts and an irregular distribution of dust and young starforming regions in their inner parts, their peculiar morphology is maintained over a range of spatial scales and surface brightnesses. As such, the morphological $K$-corrections derived from this sample mostly apply to the high-SB inner regions of these galaxies. However, these are the appropriate morphological $K$-corrections to be applied to studies of high-redshift galaxies, where cosmological SB dimming similarly selects the high-SB regions as do our short HST exposures.

More statistics are needed to cover all late types and the major dynamical stages of peculiars and mergers, as these are likely the dominant population at high redshift. We will pursue such objects further through an HST Snapshot program of late-type/irregular and peculiar galaxies in Cycle 10.

\section{SUMMARY AND CONCLUSIONS}

In order to delineate the morphology of galaxies as observed in their rest-frame mid-UV, we have carried out a systematic imaging survey with $H S T$ /WFPC 2 of 37 nearby galaxies in the mid-UV filter F300W (centered at $2930 \AA$ A), as well as in the $I$ band (filter F814W centered at $8230 \AA$ ). These mid-UV images will be useful to more reliably classify the numerous faint galaxy images seen with HST in deep Iband surveys at very high redshifts $(z \simeq 1-3)$. Eleven galaxies located in the HST CVZ were also imaged in the F255W filter (centered at $2550 \AA$ ).

Our sample is carefully selected for size and surface brightness and included galaxies of small enough radius and high enough predicted mid-UV SB to be observable with HST/WFPC2 in one orbit. Together with 17 galaxies imaged in F300W available in the HST Archive, our survey covers a wide range of Hubble types and inclinations. Complementary data at other wavelengths are available for our galaxy sample: most objects have ground-based $U B V R$ images, some have IJHK images, and 15 have far-UV images from the Astro/UIT missions. Mid-UV images (2000-3200 $\mathrm{A}$ ) have been the missing ingredient thus far. 
In summary, our mid-UV imaging database shows that in galaxies where star formation is sufficiently pronounced, it can dominate the morphology from the mid-UV through the optical, resulting in very little change in morphology from the UV to the red. However, when stellar populations older than about 1 Gyr produce most of the optical light, we see changes in morphology between the optical, where these stars tend to produce relatively regular structures as a result of the effects of orbital mixing within the galaxies, and the mid-UV, where younger stars are still at the location that reflects the distribution of their original birthplaces. An additional complication is introduced by the presence of dust obscuration. Dust lanes or clouds that are nearly transparent in the visible can be opaque in the mid-UV, thereby changing the apparent morphology.

As a function of intrinsic galaxy type, our first qualitative results from this Cycle 9 project can be summarized as follows:

1. Early-type galaxies ( $T=-6$ to 0 ) show a significant change in SB in going from the mid-UV to the red, reflecting their relative paucity of a young stellar population. Some early types appear rather dim in the mid-UV as a result of significant central dust lanes, while others show mostly a small blue nuclear feature in the mid-UV. Three elliptical galaxies become nearly point sources in the mid-UV (e.g., dominated by LINERs, Seyfert galaxies). This is in part due to our sample selection, which required the objects to have high (predicted) mid-UV SB. However, it leads us to wonder to what extent the apparently strong cosmological evolution of weak AGNs in early-type galaxies is due to the morphological $K$-correction plus SB dimming, which causes the surrounding UV-faint early-type host to no longer be visible at higher redshifts. In summary, high-SB early-type galaxies in the optical show a variety of morphologies in the mid-UV that can lead to a different morphological classification, although not necessarily always as later type. The often rather peculiar mid-UV morphology of early-type galaxies is generally quite different than that of the real late-type galaxies as seen in the mid-UV, as discussed below.

2. Mid-type spiral galaxies $(\mathrm{T}=1-5)$ and star-forming galaxies can appear as later morphological types in the midUV, as Astro/UIT has shown primarily in the far-UV. About half of the mid-type spirals in the optical appear as later morphological types in the mid-UV, but not all midtype spiral galaxies do look dramatically different in the mid-UV. One mid-type spiral, NGC 6782 (§ 3.6), shows a spectacular resonance ring full of hot stars in the $\mathrm{F} 255 \mathrm{~W}$ and F300W filters. The mid-UV images show a considerable range in the scale and $\mathrm{SB}$ of individual star-forming regions. A comparison of F300W to F814W images yields good sensitivity to dust features. Almost without exception, the midtype spirals in our sample have their small nuclear bulges bisected by a dust lane, which is often connected to the inner spiral arm structure.

3. The late-type, irregular, peculiar, and merging galaxies $(\mathrm{T}=6-11)$ in our sample show diverse properties in the mid-UV. The majority of these galaxies show an F300W morphology that is similar to that in F814W, but with important differences due to recognizable dust features absorbing the F300W light and hot stars, star clusters, or star formation ridges that are better visible in the mid-UV. Less than a third of galaxies classified as late-type in the optical show significantly different structures in the mid-UV to result in a different classification.

In conclusion, our HST mid-UV survey of nearby galaxies suggests that it is more likely to misclassify true earlyto mid-type galaxies in the rest-frame mid-UV as later types, while known late-type galaxies are less likely to be misclassified (as earlier types) in the mid-UV. This is because truly late-type galaxies are dominated by young and hot stars in all filters from the mid-UV to the red and so have to first order the same morphology and a very small morphological $K$-correction in general. However, early-type galaxies (elliptical galaxies and early-type spirals) can, although do not have to, look significantly different when one goes from the rest-frame mid-UV to the optical-red part of the spectrum.

Classification of faint galaxies in the rest-frame mid-UV will thus likely result in some fraction of early- to mid-type galaxies being misclassified as later types, probably a larger fraction than vice versa. However, it is unlikely that this morphological $K$-correction can explain all of the faint blue galaxy excess as misclassified earlier type galaxies, and the morphological $K$-correction cannot explain the slight excess of early- to mid-type galaxies at faint magnitudes $(B \gtrsim 24$ mag) with respect to passively evolving models, as found by Odewahn et al. (1996) and Cohen et al. (2002), since the main misclassification error goes in the opposite direction. Instead, our mid-UV survey seems to support the conclusion of Cohen et al. (2002) that the number of faint galaxies is larger than the nonevolving predictions for all galaxy types, but more significantly so for the later types. Cohen et al. (2002) give a possible explanation of this finding in terms of hierarchical formation models in a $\Lambda$-dominated universe.

To address these issues further quantitatively, we will in a sequel paper use the Fourier decomposition method of Odewahn (1995) and Odewahn et al. (2002), where we will quantify the morphological $K$-correction based on each galaxy's mid-UV photometric parameters as a function of rest-frame wavelength and observed type. Our goal is to use the current $H S T$ mid-UV sample to quantify the morphological $K$-correction and apply it to a large complete sample of faint $H S T$ galaxies with known photometric redshifts to get reliable and consistent rest-frame classifications. These will then be used to compute the redshift distribution as a function of accurately determined morphological type, which will help delineate the formation and evolution of galaxies along the Hubble sequence (see Driver et al. 1998).

The US authors acknowledge support from NASA grants GO 8645.* and AR-8765.*, and R. S. d. J. acknowledges support from NASA through Hubble Fellowship grant HF01106.01-A, awarded by STScI, which is operated by AURA for NASA under contract NAS 5-26555. R. A. W., C. A. C., and S. C. O. acknowledge support from NASA ADP grant NAG-6740. C. A. C. and V. A. T. acknowledge support from a NASA Space Grant Graduate Fellowship at ASU. This research has made use of the NASA/IPAC Extragalactic Database (NED), which is operated by the Jet Propulsion Laboratory, California Institute of Technology (Caltech), under contract with NASA, and has used NASA's Astrophysics Data System Bibliographic Services. The Second Palomar Observatory Sky Survey (POSS II) was made by Caltech with funds from the NSF, NASA, the National Geographic Society, the Sloan Foundation, the 
Samuel Oschin Foundation, and the Eastman Kodak Corporation. The Oschin Schmidt Telescope is operated by Caltech and Palomar Observatory. We thank Tony Roman and the STScI staff for their excellent help in getting these HST observations scheduled. We thank the staff of the Vatican Advanced Technology Telescope, in particular Richard Boyle, Chris Corbally, and Matt Nelson, for their excellent help in obtaining a large fraction of the ground-based images presented in this paper. We also thank Seth Cohen for advice in the data reduction, Arthur Code for a careful reading of the manuscript and for fruitful discussions, and Peter Strittmatter and the University of Arizona for their hospitality during a working visit. We thank the referee, Rob Kennicutt, for a careful and helpful review of the manuscript and for suggesting a new way to publish a paper like the current one.
Abraham, R. G., Tanvir, N. R., Santiago, B. X., Ellis, R. S., Glazebrook, K., \& van den Bergh, S. 1996, MNRAS, 279, L47

Abraham, R. G., et al. 1999, MNRAS, 308, 569

Allam, S., Assendorp, R., Longo, G., Braun, M., \& Richter, G. 1996, A\&AS, 117,39

Alonso-Herrero, A., Rieke, G. H., Rieke, M. J., \& Scoville, N. Z. 2000, ApJ, 532, 845

Arp, H. C. 1966, ApJS, 14, 1

Bahcall, J. N., Kirhakos, S., Saxe, D. H., \& Schneider, D. P. 1997, ApJ, 479,642

Bahcall, J. N., Kirhakos, S., \& Schneider, D. P. 1995, ApJ, 450, 486 1996, ApJ, 457, 557

Balick, B., \& Heckman, T. 1981, A\&A, 96, 271

Barnes, J. E. 2002, MNR AS, 333, 481

Barnes, J. E., \& Hernquist, L. 1992, ARA\&A, 30, 705

Beck, S. C., Turner, J. L., Ho, P. T. P., Lacy, J. H., \& Kelly, D. M. 1996, ApJ, 457, 610

Beichman, C., Neugebauer, G., Habing, H. J., Clegg, P. E., \& Chester, T. J. 1988, IRAS Catalogs and Atlases Explanatory Supplement, NASA RP1190, Vol. 1 (Washington, DC: GPO)

Bertin, E., \& Arnouts, S. 1996, A\&AS, 117, 393

Biretta, J., et al. 2001, WFPC2 Instrument Handbook, Version 6.0 (Baltimore: STScI)

Böker, T., Laine, S., van der Marel, R. P., Sarzi, M., Rix, H.-W., Ho, L. C., \& Shields, J. C. 2001, BAAS, 199, 07.05

Bohlin, R. C., et al. 1991, ApJ, 368, 12

Bouwens, R. J., Broadhurst, T., \& Silk, J. 1998, ApJ, 506, 579

Bowyer, S., Sasseen, T. P., Wu, X., \& Lampton, M. 1995, ApJS, 96, 461

Boyle, B. J., Shanks, T., Croom, S. M., Smith, R. J., Miller, L., Loaring, N., \& Heymans, C. 2000, MNRAS, 317, 1014

Boyle, B. J., \& Terlevich, R. J. 1998, MNRAS, 293, L49

Brinchmann, J., et al. 1998, ApJ, 499, 112

Brown, T. M., Bowers, C. W., Kimble, R. A., \& Ferguson, H. C. 2000, ApJ, 529, L89

Bruzual, A. G., \& Charlot, S. 1993, ApJ, 405, 538

Bryant, P. M., \& Scoville, N. Z. 1999, AJ, 117, 2632

Burgarella, D., Buat, V., Donas, J., Milliard, B., \& Chapelon, S. 2001, A\&A, 369, 421

Burstein, D., Bertola, F., Buson, L. M., Faber, S. M., \& Lauer, T. R. 1988, ApJ, 328, 440

Buta, R., Alpert, A. J., Cobb, M. L., Crocker, D. A., \& Purcell, G. B. 1998, AJ, 116, 1142

Buta, R., \& Purcell, G. B. 1998, AJ, 115, 484

Buta, R., Purcell, G. B., Cobb, M. L., Crocker, D. A., Rautiainen, P., \& Salo, H. 1999, AJ, 117, 778

Buta, R., Purcell, G. B., \& Crocker, D. A. 1996, AJ, 111, 983

Buta, R., Ryder, S. D., Madsen, G. J., Wesson, K., Crocker, D. A., \& Combes, F. 2001, AJ, 121, 225

Buta, R., Treuthardt, P. M., Byrd, G. G., \& Crocker, D. A. 2000, AJ, 120, 1289

Caldwell, N., \& Phillips, M. M. 1989, ApJ, 338, 789

Calzetti, D., Bohlin, R. C., Kinney, A. L., Storchi-Bergmann, T., \& Heckman, T. M. 1995, ApJ, 443, 136

Calzetti, D., Conselice, C. J., Gallagher, J. S., III, \& Kinney, A. L. 1999, AJ, 118, 797

Calzetti, D., Meurer, G. R., Bohlin, R. C., Garnett, D. R., Kinney, A. L., Leitherer, C., \& Storchi-Bergmann, T. 1997, AJ, 114, 1834

Carruthers, G. R., Opal, C. B., \& Heckathorn, H. M. 1978, ApJ, 225, 346

Casoli, F., Willaime, M.-C., Viallefond, F., \& Gerin, M. 1999, A\&A, 346, 663

Code, A. D., \& Welch, G. A. 1982, ApJ, 256, 1

Cohen, J. M., Hogg, D. W., Blandford, R., Cowie, L. L., Hu, E., Songaila, A., Shopbell, P., \& Richberg, K. 2000, ApJ, 538, 29

Cohen, S. H., Windhorst, R. A., Chiarenza, C. A. T., Odewahn, S. C., \& Driver, S. P. 2002, AJ, submitted

Conselice, C. J., Gallagher, J. S., Calzetti, D., Homeier, N., \& Kinney, A. 2000, AJ, 119,79

Cornett, R. H., et al. 1994, ApJ, 426, 553

Cowie, L. L., Hu, E. M., \& Songaila, A. 1995, Nature, 377, 603

Crowther, P. A., Beck, S. C., Willis, A. J., Conti, P. S., Morris, P. W., \& Sutherland, R. S. 1999, MNRAS, 304, 654

Dalcanton, J. J., \& Shectman, S. A. 1996, ApJ, 465, L9

de Grijs, R., O'Connell, R. W., \& Gallagher J. S. 2001, AJ, 121, 768

de Grijs, R., \& Peletier, R. F. 1997, A\&A, 320, L21 de Grijs, R., Peletier, R. F., \& van der Kruit, P. C. 1997, A\&A, 327, 966 de Jong, R. S., \& van der Kruit, P. C. 1994, A\&AS, 106, 451

de Vaucouleurs, G., de Vaucouleurs, A., Corwin, H., Buta, R., Paturel, G., \& Fouqué, P. 1991, Third Reference Catalogue of Bright Galaxies (New York: Springer)

Dickinson, M. E., et al. 2001, in Proc. 257 of the XIXth Moriond Astrophysics Meeting, Building Galaxies: From the Primordial Universe to the Present, ed. F. Hammer, T. X. Thuan, V. Cayatte, B. Guiderdoni, \& J. Tranh Than Van (Paris: Editions Frontieres), 257

Disney, M. J. 1976, Nature, 263, 573

Donas, J., Milliard, B., \& Laget, M. 1995, A\&A, 303, 661

Downes, D., \& Solomon, P. M. 1998, ApJ, 507, 615

Driver, S., Fernandez-Soto, A., Couch, W., Odewahn, S., Windhorst, R., Phillipps, S., Lanzetta, K., \& Yahil, A. 1998, ApJ, 496, L93

Driver, S. P. 1999, ApJ, 526, L69

Driver, S. P., Windhorst, R. A., \& Griffiths, R. E. 1995a, ApJ, 453, 48

Driver, S. P., Windhorst, R. A., Ostrander, E. J., Keel, W. C., Griffiths, R. E., \& Ratnatunga, K. U. 1995b, ApJ, 449, L23

Eichendorf, W., \& Nieto, J.-L. 1984, A\&A, 132, 342

Ellis, R. S., Colless, M., Broadhurst, T., Heyl, J., \& Glazebrook, K. 1996, MNRAS, 280, 235

Eskridge, P. B., et al. 2000, AJ, 119, 536 .2002, ApJS, submitted

Fasano, G., \& Bettoni, D. 1994, AJ, 107, 1649

Filippenko, A. V. 1985, ApJS, 57, 503

Frei, Z., Guhathakurta, P., Gunn, J. E., \& Tyson, J. A. 1996, AJ, 111, 174

Frogel, J. A., Quillen, A. C., \& Pogge, R. W. 1996, in New Extragalactic Perspectives in the New South Africa, Astrophysics and Space Science Library, Vol. 209, ed. D. L. Block \& J. M. Greenberg (Dordrecht: Kluwer), 65

Gallagher, J. S., Conselice, C. J., Homeier, N., \& the WFPC2 Investigation Definition Team. 2000, in ASP Conf. Ser. 197, Dynamics of Galaxies: from the Early Universe to the Present, ed. F. Combes, G. A. Mammon, $\&$ V. Charmandaris (San Francisco: ASP), 309

Gallagher, J. S., Hunter, D. A., \& Bushouse, H. 1989, AJ, 97, 700

Gaposchkin, C. P. 1936, ApJ, 83, 173

Giavalisco, M., Livio, M., Bohlin, R. C., Macchetto, F. D., \& Stecher, T. P. 1996, AJ, 112, 369

Glazebrook, K., Ellis, R. E., Santiago, B., \& Griffiths, R. E. 1995, MNRAS, 275, L19

Gorjian, V. 1996, AJ, 112, 1886

Greggio, L., \& Renzini, A. 1990, ApJ, 364, 35

Griffiths, R. E., et al. 1994, ApJ, 435, L19

Hawarden, T. G., van Woerden, H., Mebold, U., Goss, W. M., \& Peterson, B. A. 1979, A\&A, 76, 230

Hibbard, J. E., \& Vacca, W. D. 1997, AJ, 114, 1741

Hibbard, J. E., \& van Gorkom, J. H. 1996, AJ, 111, 655

Hibbard, J. E., \& Yun, M. S. 1999a, AJ, 118, 162 1999b, ApJ, 522, L93

Hill, J. K., et al. 1992, ApJ, 395, L37

Holtzman, J. A., Burrows, C. J. Casertano, S., Hester, J. J., Trauger, J. T., Watson, A. M., \& Worthey, G. 1995, PASP, 107, 1065

Hubble, E. P., \& Lundmark, K. 1922, PASP, 34, 292

Hunter, D. A., O'Connell, R. W., \& Gallagher J. S. 1994, AJ, 108, 84

Israel, F., de Boer, K. S., \& Bosma, A. 1986, A\&AS, 66, 117

Jansen, R. A., Knapen, J. H., Beckman, J. E., Peletier, R. F., \& Hes, R. 1994, MNRAS, 270, 373

Keel, W. C. 1996a, AJ, 111, 696 1996b, ApJS, 106, 27 2000, BAAS, 197, 37.01

Keel, W. C., \& Weedman, D. W. 1978, AJ, 83,

Keel, W. C., \& Windhorst, R. A. 1993, AJ, 106, 455

Kent, S. M. 1985, ApJS, 59, 115

Khachikian, E. 1974, ApJ, 192, 581

Kinney, A. L., Bohlin, R. C., Calzetti, D., Panagia, N., \& Wyse, R. F. G. 1993, ApJS, 86, 5

Kirhakos, S., Bahcall, J. N., Schneider, D. P., \& Kristian, J. 1999, ApJ, 520, 67

Knapen, J. H., \& Beckman, J. E. 1996, MNRAS, 283, 251

Knapen, J. H., Laine, S., Yates, J. A., Robinson, A., Richards, A. M., S., Doyon, R. \& Nadeau, D. 1997, ApJ, 490, L29

Knapen, J. H., Shlosman, I., \& Peletier, R. F. 2000, ApJ, 529, 93

Kobulnicky, H. A., \& Skillman, E. D. 1995, ApJ, 454, L121

Koo, D. C., \& Kron, R. G. 1988, ApJ, 325, 92 
Kregel, M., \& Sancisi, R. 2001, A\&A, 376, 59

Kuchinski, L. E., Madore, B. F., Freedman, W. L., \& Trewhella, M. 2001, AJ, 122,729

Kuchinski, L. E., Terndrup, D. M., Gordon, K. D., \& Witt, A. N. 1998, AJ, 115, 1438

Kuchinski, L. E., et al. 2000, ApJS, 131, 441

Lauberts, A., \& Valentijn, E. A. 1989, The Surface Photometry Catalogue of the ESO-Uppsala Galaxies (Garching: ESO)

Laurent-Muehleisen, S. A., Kollgaard, R. I., Ryan, P. J., Feigelson, E. D., Brinkmann, W., \& Siebert, J. 1997, A\&AS, 122, 235

Lilly, S. J., et al. 1998, ApJ, 500, 75

Magorrian, J., et al. 1998, AJ, 115, 2285

Majewski, S. R., Hereld, M., Koo, D. C., Illingworth, G. D., \& Heckman, T. M. 1993, ApJ, 402, 125

Maoz, D., Filippenko, A. V., Ho, L. C., Macchetto, F. D., Rix, H.-W., \& Schneider, D. P. 1996, ApJS, 107, 215

Marcum, P. M., et al. 2001, ApJS, 132, 129

Marlowe, A. T., Heckman, T. M., Wyse, R. F. G., \& Schommer, R. 1995, ApJ, 438, 563

Matthews, L. D., \& Gallagher, J. S. 1997, AJ, 114, 1899

Matthews, L. D., et al. 1999, AJ, 118, 208

Meurer, G. R., Heckman, T. M., Leitherer, C., Kinney, A., Robert, C., \& Garnett, D. R. 1995, AJ, 110, 2665

Milliard, B., Donas, J., Laget, M., Armand, C., \& Vuillemin, A. 1992, A\&A, 257, 24

Moffat, A. F. J. 1969, A\&A, 3, 455

Mulder, P. S., \& van Driel, W. 1996, A\&A, 309, 403

Mulder, P. S., van Driel, W., \& Braine, J. 1995, A\&A, 300, 687

Naab, T., \& Burkert, A. 2001, ApJ, 555, L91

Neuschaefer, L. W., \& Windhorst, R. A. 1995, ApJS, 96, 371

Nordgren, T. E., Chengalur, J. N., Salpeter, E. E., \& Terzian, Y. 1997, AJ, 114,77

O'Connell, R. W. 1999, ARA\&A, 37,603

O'Connell, R. W., et al. 1992, ApJ, 395, L45

Odewahn, S. C. 1995, PASP, 107, 770

Odewahn, S. C., Burstein, D., \& Windhorst, R. A. 1997, AJ, 114, 2219

Odewahn, S. C., Cohen, S. H., Windhorst, R. A., \& Phillip, S. 2002, ApJ, 568,539

Odewahn, S. C., Windhorst, R. A., Driver, S. P., \& Keel, W. C. 1996, ApJ, 472, L13

Osterbrock, D. 1977, ApJ, 215, 733

Pascarelle, S. M., Windhorst, R. A., \& Keel, W. C. 1998, AJ, 116, 2659

Pascarelle, S. M., Windhorst, R. A., Keel, W. C., \& Odewahn, S.C. 1996, Nature, 383,45

Perlman, E. S., Padovani, P., Giommi, P., Sambruna, R., Jones, L. R., Tzioumis, A., \& Reynolds, J. 1998, AJ, 115, 1253

Phookun, B., Mundy, L. G., Teuben, P., \& Wainscoat, R. J. 1992, ApJ, 400, 516

Quillen, A. C., McDonald, C., Alonso-Herrero, A., Lee, A., Shaked, S., Rieke, M. J., \& Rieke, G. H. 2001, ApJ, 547, 129

Radecke, H. D. 1997, A\&A, 319, 18

Ravindranath, S., Ho, L. C., Peng, C. Y., Filippenko, A. V., \& Sargent, W. L. W. 2001, AJ, 122, 653

Regan, M. W., \& Mulchaey, J. S. 1999, AJ, 117, 2676

Roche, N., Ratnatunga, K., Griffiths, R. E., \& Im, M. 1997, MNRAS, 288, 200

Ruiz, J. R., Crenshaw, D. M., Kraemer, S. B., Bower, G. A., Gull, T. R., Hutchings, J. B., Kaiser, M. E., \& Weistrop, D. 2001, AJ, 122, 2961

Sandage, A., Saha, A., Tammann, G. A., Labhardt, L., Schwengeler, H., Panagia, N., \& Macchetto, F. D. 1994, ApJ, 423, L13

Sanders, D. B., Soifer, B. T., Elias, J. H., Madore, B. F., Matthews, K., Neugebauer, G., \& Scoville, N. Z. 1988, ApJ, 325, 74
Schwarzkopf, U., \& Dettmar, R.-J. 2000, A\&A, 361, 451

Schweizer, F. 1996, AJ, 111, 109

Schweizer, F., Miller, B. W., Whitmore, B. C., \& Fall, S. M. 1996, AJ, 112, 1839

Scoville, N. Z., et al. 2000, AJ, 119, 991

Sersic, J. L., Carranza, G., \& Pastoriza, M. 1972, Ap\&SS, 19, 469

Seyfert, C. K. 1943, ApJ, 97, 28

Simard, L., et al. 1999, ApJ, 519, 563

Smith, A. M., et al. 2001, ApJ, 546, 829

Smith, D. A., Herter, T., Haynes, M. P., Beichman, C. A., \& Gautier, T. N. 1996a, ApJS, 104, 217

Smith, D. A., et al. 1996b, ApJ, 473, L2

Stecher, T. P., Bohlin, R. C., Hill, J. K., \& Jura, M. A. 1982, ApJ, 255, L99

Stecher, T. P., et al. 1997, PASP, 109, 584

Storchi-Bergmann, T., Kinney, A. L., \& Challis, P. 1995, ApJS, 98, 103

Strickland, D. K., \& Stevens, I. R. 1999, MNRAS, 306, 43

Surace, J. A., \& Sanders, D. B. 2000, AJ, 120, 604

Surace, J. A., Sanders, D. B., \& Evans, A. S. 2000, ApJ, 529, 170

Taylor, G. B., Vermeulen, R. C., Readhead, A. C. S., Pearson, T. J., Henstock, D. R., \& Wilkinson, P. N. 1996, ApJS, 107, 37

Toomre, A. 1977, in The Evolution of Galaxies and Stellar Populations, ed. B. M. Tinsley \& R. B. Larson (New Haven: Yale Univ. Press), 401

Treyer, M. A., Ellis, R. S., Milliard, B., Donas, J., \& Bridges, T. J. 1998, MNRAS, 300, 303

Turner, J. L., Beck, S. C., \& Ho, P. T. 2000, ApJ, 532, L109

Turner, J. L., Beck, S. C., \& Hurt, R. L. 1997, ApJ, 474, L11

Turner, J. L., Ho, P. T., \& Beck, S. C. 1998, AJ, 116, 1212

van Albada, T. S. 1982, MNRAS, 201, 939

Vogt, N. P., et al. 1997, ApJ, 479, L121

Wang, Z., Schweizer, F., \& Scoville, N. Z. 1992, ApJ, 396, 510

White, R. L., \& Becker, R. H. 1992, ApJS, 79, 331

Whitmore, B. C., Schweizer, F., Leitherer, C., Borne, K., \& Robert, C. 1993, AJ, 106, 1354

Williams, R. E., et al. 1996, AJ, 112, 1335

. 2000, AJ, 120, 2735

Windhorst, R. A., Franklin, B. E., \& Neuschaefer, L. W. 1994a, PASP, 106,798

Windhorst, R. A., Gordon, J. M., Pascarelle, S. M., Schmidtke, P. C., Keel, W. C., Burkey, J. M., \& Dunlop, J. S. 1994b, ApJ, 435, 577

Windhorst, R. A., Keel, W. C., \& Pascarelle, S. M. 1998a, ApJ, 494, L27

Windhorst, R. A., Odewahn, S. C., Burg, C., Cohen, S. H., \& Waddington, I. 1999, Ap\&SS, 269, 243

Windhorst, R. A., Pascarelle, S. M., Odewahn, S. C., Cohen, S. H., Burg, C., Keel, W. C., \& Driver, S. P. 1998b, in Proceedings of the STScI Symposium, The Hubble Deep Field, ed. M. Livio, S. M. Fall, \& P. Madau (Cambridge: Cambridge Univ. Press), 481

Windhorst, R. A., et al. 1994c, AJ, 107, 930

. 1996, in Proceedings of the Max Planck Workshop on Galaxies in the Young Universe, Springer Lecture Notes in Physics, Vol. 463, ed H. Hippelein, K. Meisenheimer, \& H.-J. Roeser (Berlin: Springer), 265 1997, in The Ultraviolet Universe at Low and High Redshift: Probing the Progress of Galaxy Evolution, Vol. 408, ed. W. H. Waller, M. N. Fanelli, J. E. Hollis, \& A. C. Danks (New York: AIP), 242

Witt, A. N., \& Gordon, K. D. 1996, ApJ, 463, 681

Wright, A. E., Griffith, M. R., Hunt, A. J., Troup, E., Burke, B. F., \& Ekers, R. D. 1996, ApJS, 103, 145

Yun, M. S., \& Hibbard, J. E. 2001, ApJ, 550, 104

Yun, M. S., \& Scoville, N. Z. 1995, ApJ, 451, L45

Zeltwanger, T., Comins, N. F., \& Lovelace, R. V. E. 2000, ApJ, 543, 669

Zepf, S. E., Ashman, K., English, J., Freeman, K. C., \& Sharples, R. M. 1999, AJ, 118, 752 